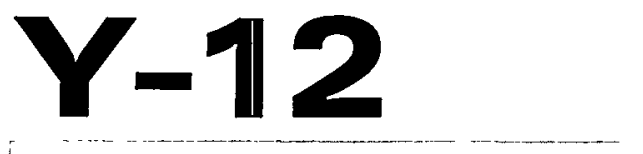

OAK RIDGE Y-12

\section{PLANT}

LOCKHEED WATTHY

\section{RECEIVED \\ OCT 011999 \\ OSTI}

Programming Enhancements for Low Temperature Thermal Decomposition Workstation

\author{
R. E. Igou \\ Fabrication Systems Department \\ Development Division
}

October 1998
Oak Ridge Y-12 Plant

P. O. Box 2009,

Oak Ridge, TN 37831-8169 managed by

LOCKHEED MARTIN ENERGY SYSTEMS, INC.

for the

U.S. DEP ARTMENT OF ENERGY

under contract DE-AC05-84OR21400

MANAGED BY

LOCKHEED MARTIN ENERGY SYSTEMS, INC.

FOR THE UNITED STATES

DEPARTMENT OF ENERGY

UCN-13672 (26 6-95) 


\section{DISCLAIMER}

This report was prepared as an account of work sponsored by an agency of the United States Government. Neither the United States Government nor any agency thereof, nor any of their employees, makes any warranty, express or implied, or assumes any legal liability or responsibility for the accuracy, completeness, or usefulness of any information, apparatus, product, or process disclosed, or represents that its use would not infringe privately owned rights. Reference herein to any specific commercial product, process, or service by trade name, trademark, manufacturer, or otherwise, does not necessarily constitute or imply its endorsement, recommendation, or favoring by the United States Government or any agency thereof. The views and opinions of authors expressed herein do not necessarily state or reflect those of the United States Government or any agency thereof. 


\section{DISCLAIMER}

Portions of this document may be illegible in electronic image products. Images are produced from the best available original document. 


\title{
Programming Enhancements for Low Temperature Thermal Decomposition Workstation
}

\author{
R. E. Igou \\ Fabrication Systems Department \\ Development Division
}

October 1998

\author{
Prepared by the \\ Oak Ridge Y-12 Plant \\ P. O. Box 2009, \\ Oak Ridge, TN 37831-8169 \\ managed by \\ LOCKHEED MARTIN ENERGY SYSTEMS, INC. \\ for the \\ U.S. DEPARTMENT OF ENERGY \\ under contract DE-AC05-84OR21400
}




\section{TABLE OF CONTENTS}

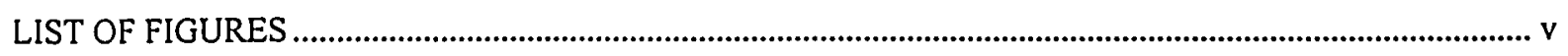

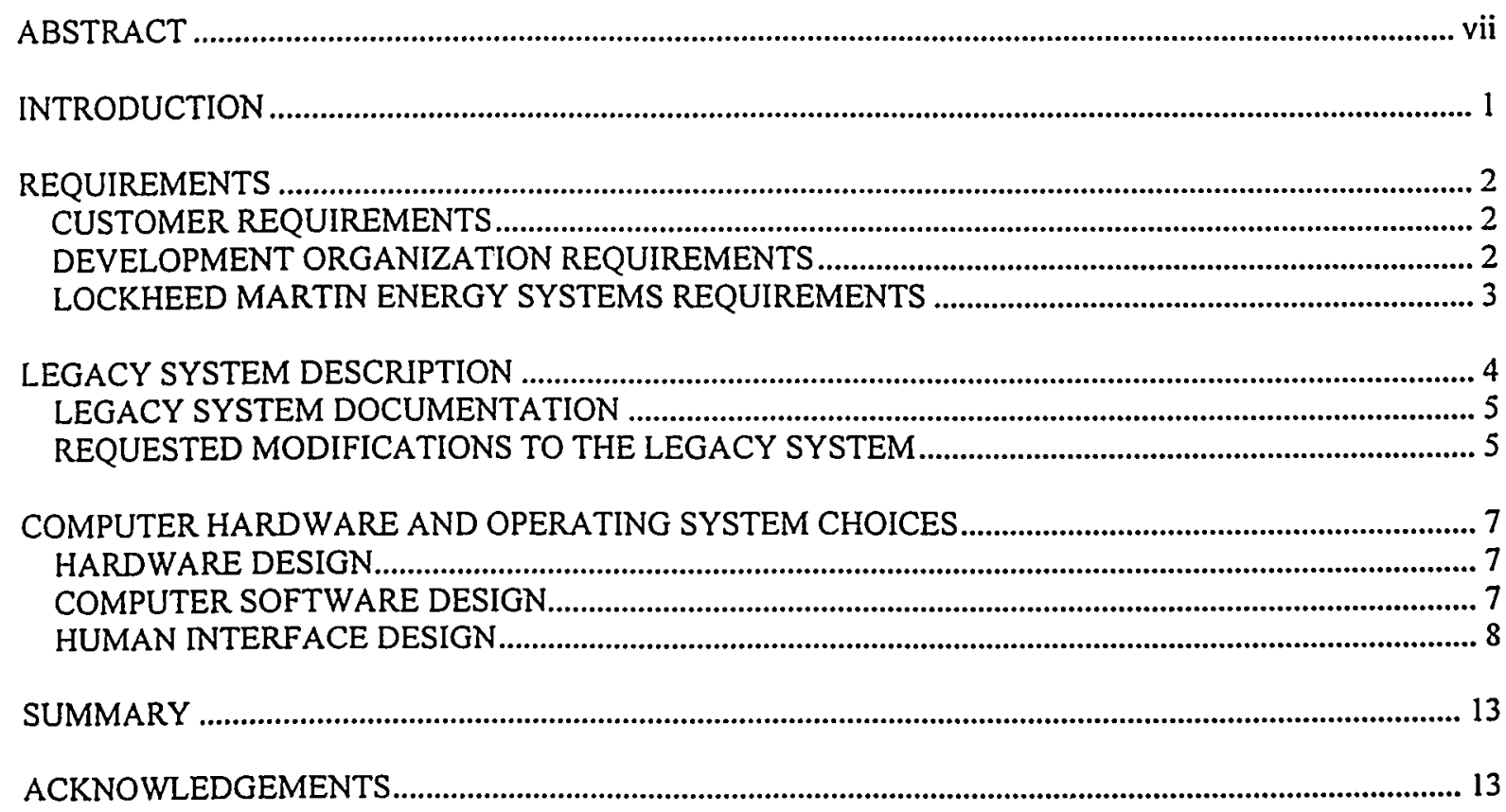

APPENDIX A: INVENTORY OF LEGACY LTTD WORKSTATION DOCUMENTATION ........................ 14

APPENDIX B: SOURCE CODE FOR LEGACY THERMAL DECOMPOSITION SOFTWARE ................... 17

APPENDIX C: FLOWCHART FOR LEGACY THERMAL DECOMPOSITION SOFTWARE ...................... 34

APPENDIX D: SOURCE CODE FOR LEGACY MANIFOLD VOLUME CALIBRATION SOFTWARE.... 38

APPENDIX E: FLOWCHART FOR MODIFIED THERMAL DECOMPOSITION SOFTWARE .................. 48

APPENDIX F: HARDWARE DESIGN FOR ENHANCED LTTD WORKSTATION .................................... 52 


\section{LIST OF FIGURES}

Figure 1. Legacy system vacuum manifold .............................................................................. 4

Figure 2. Enhanced LTTD workstation sample front panel ........................................................... 8



Figure 4. Manual mode with trend display .............................................................................. 10

Figure 5. Automatic mode data/report generation ........................................................................... 11 


\begin{abstract}
This report describes a new control-and-measurement system design for the Oak Ridge Y-12 Plant's Low Temperature Thermal Decomposition (LTTD) process. The new design addresses problems with system reliability stemming from equipment obsolescence and addresses specific functional improvements that plant production personnel have identified, as required.

The new design will also support new measurement techniques, which the Y-12 Development Division has identified for future operations. The new techniques will function in concert with the original technique so that process data consistency is maintained.
\end{abstract}




\section{INTRODUCTION}

The Oak Ridge Y-12 Plant's Disassembly and Storage Organization (DSO) uses a process called Low Temperature Thermal Decomposition (LTTD). The existing LTTD process workstations, hereafter referred to as the legacy system, need to be redesigned. DSO's primary reason for the new design is a serious reliability problem due to the fact that the controllers are based on computer systems that have become obsolete over the past decade. A second reason is that the existing software for the LTTD legacy system exhibits a number of operational problems, which DSO wants to eliminate. An example of these problems is the unfriendly user interface typical of older computer systems. A third reason is that DSO has accumulated a list of functional improvements that are desired for the LTTD workstation software but cannot practically be implemented on the older computer platform.

The Y-12 Development Division also wants a redesigned controller for the LTTD process because the division has long proposed improvements to the LTTD measurements that the LTTD process performs. A newly designed LTTD that meets DSO's requirements is seen as a platform for developing an improved LTTD measurement process while maintaining consistency with the da 


\section{REQUIREMENTS}

\section{CUSTOMER REQUIREMENTS}

- DSO has imposed a number of requirements and constraints on the design solution.

- A new LTTD workstation shall be constructed to be used as a test bed for the design solution. (The new workstation with the implementation of the design solution shall be referred to as the Enhanced LTTD (ELTTD) workstation).

- The ELTTD workstation shall be demonstrated to be functionally acceptable before deployment.

- The ELTTD workstation shall be demonstrated to be reliable before deployment.

- The design solution shall be implementable as an upgrade to the legacy LTTD workstations. The goal is to implement the upgrade with less than a week downtime.

- The functional behavior of the legacy LTTD workstations is to be considered the baseline for the functional behavior of the ELTTD workstation. DSO provides specific exceptions.

- The design solution shall use a widely accepted, commodity-priced computer system that has an expected product-family lifetime in excess of 7 years.

- The design solution shall use widely accepted, commodity-priced operating system software that has an expected product-family lifetime in excess of 7 years.

- The design solution shall use support software (compilers, interpreters, etc.) that is widely accepted for this type of application and that has an expected product-family lifetime in excess of 7 years.

\section{DEVELOPMENT DIVISION REQUIREMENTS}

Development Division has also imposed requirement constraints on the design solution.

- The design solution shall be flexible enough to support experiments on the LTTD me rement process.

- The design solution shall be flexible enough to support additional LTTD measurement tec ues to be defined at some future date.

- The design solution shall support the existing LTTD measurement techniques to ensure cont. : ity of data. 


\section{LOCKHEED MARTIN ENERGY SYSTEMS REQUIREMENTS}

The U.S. Department of Energy (DOE) and the Y-12 Plant place requirements and constraints on the design in the form of software quality assurance requirements. Year 2000 compliance of all system components is a particular consideration this close to the millennium. 


\section{LEGACY SYSTEM DESCRIPTION}

Figure 1 shows the legacy system vacuum manifold.

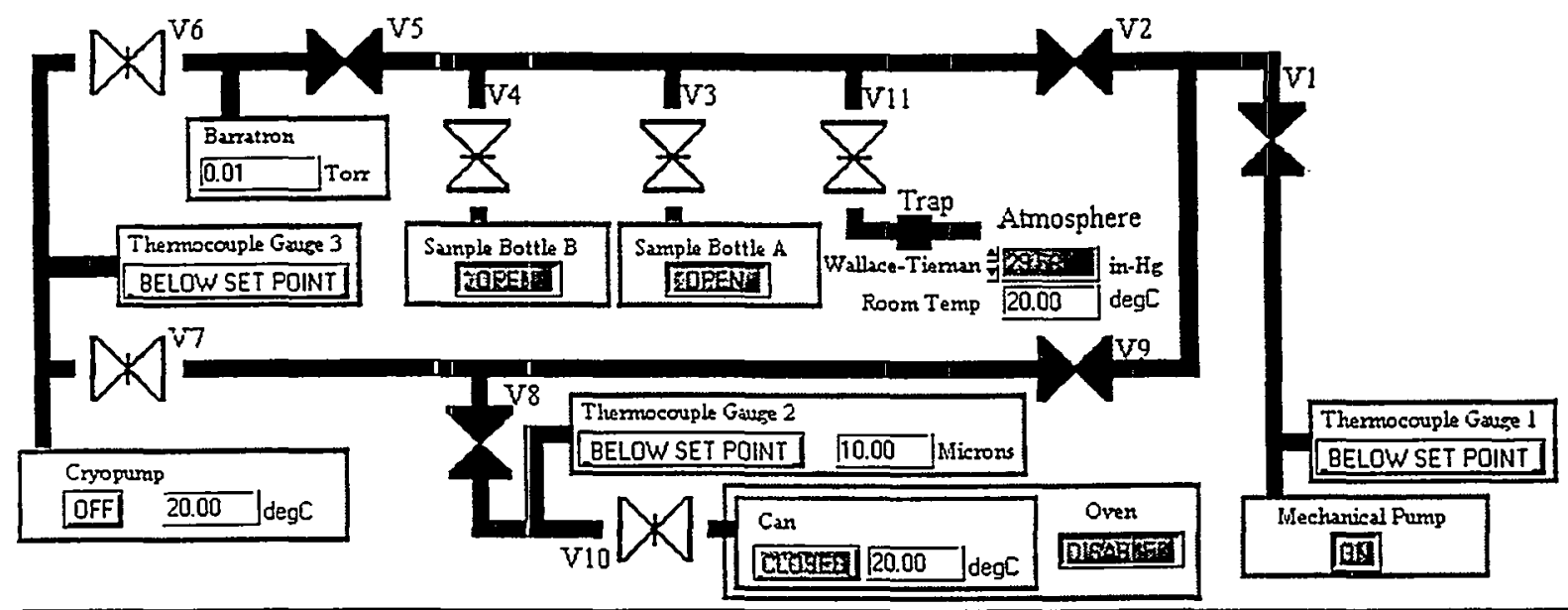

Fig. 1. Legacy system vacuum manfiold

The legacy system has two vacuum ports for sample collection bottles and one port for the sample that will be placed in an oven. A mechanical pump is used to rough down the vacuum system and a cryopump is used to capture gas given off when the sample is heated. Two thermocouple gauges (TG1 \& TG3) open and close relays when pressure is above or below a setpoint. An additional thermocouple gauge (TG2) has an analog pressure readout to monitor pressure on the manifold side of the sample port. A Baratron head provides precision measurements of pressure during sample gas collection. Thermocouple gauges are used to measure the temperatures of the manifold, oven, and cryopump.

Operation of the system is conceptually simple. The mechanical pump roughs down the vacuum and the system is then checked for leaks. The oven heats the sample, and the cryopump captures the resulting gas. When all of the gas has been captured, the cryopump is allowed to regenerate and the gas is transferred to the sample collection bottles.

A Hewlett-Packard industrial computer running the HPBasic Interpreter and a Basic program wr ten specially for the system automates the operation of the system. The valves, contactors for the oven and cryopump, and sensors for pressure and temperature are wired to conditioning modules in a Hewlett-Packard data acquisition module. The data acquisition module is connected to the computer through a General Purpose Interface Bus (GPIB) cable. 


\section{LEGACY SYSTEM DOCUMENTATION}

The documentation folder for the existing LTTDs was inventoried to capture the design of the legacy system. The items in the folder were divided into 14 categories, which are in Appendix A. The key items were found to be

- LTTD program source code,

- LTTD program flow chart,

- manifold volume calibration program source code.

These items (Appendices $B, C$, and D, respectively, in this report) correspond to entries 1,7 , and 9 in the inventory.

The Engineering drawings for the legacy system were also found to be important items. Copies of those drawings, Nos. I2E137698 and I2E143710, can be acquired from Y-12 Engineering records on request.

\section{REQUESTED MODIFICATIONS TO THE LEGACY SYSTEM}

DSO desired a number of modifications to the legacy system design. These changes had never been formally documented. A series of three interviews were conducted with DSO's designated representative to capture and document the desired changes. These interviews were carried out between May and June of 1997.

Two changes were desired in the vacuum manifold design:

- A turbomolecular pump was inserted to protect the manifold from oil contamination due to the roughing pump.

- All three thermocouple gauges were specified to be the same make and model to simplify system maintenance.

DSO also accepted proposed modifications to support future Development activities:

- Flanges were provided to support future connections for a mass spectrometer and standard leaks, and

- An ion gauge was incorporated.

Desired functional modifications to the legacy system software are as follows:

- The LTTD program sequence of operations to calibrate the Baratron zero reading was changed to improve the efficiency of the operation. Modifying the flowchart of the LTTD program (primarily page 3) captured this change. Appendix E presents the new flowchart.

- Operator inputs shall tolerate typing errors. (The legacy system has many operations for which extensive data entry is required. The program must be restarted at the beginning to correct typographical errors). 
- Detection of problems (such as adjustments out of calibration) after thermal decomposition has started should cause the program to flag the problem but otherwise continue. (The legacy system will quit the program if it detects any kind of problem.)

- Detection of problems, which prevent continuation of the program, should allow restart if the operator can correct the problem. (The legacy system will quit if a sample bottle fails a leak check. The legacy system must be restarted at the beginning if this occurs. Several similar cases exist.)

- Cryopump saturation should be handled by allowing the operator to force the collection sequence to completion and then reenter thermal decomposition. (The legacy system requires the operator to stop the program and manually complete the sequence if cryopump saturation occurs.)

- The enhanced system shall automatically start when power is applied. (The legacy system requires substantial manual activities to start.)

- The enhanced system shall remember its position in the sequence of operations and attempt to automatically continue in the event of a power fail/restore condition. (The legacy system does not maintain state information of any kind and cannot automatically start on power restore.)

- The enhanced system shall graphically display process data. At a minimum, three process variables (oven temperature, cryopump temperature, and sample pressure) shall be simultaneously shown on a display like a "continuous strip chart." (The legacy system cannot support graphical data display.)

- The enhanced system shall protect system shutdown and any other maintenance operation by password-only access. 


\section{COMPUTER HARDWARE AND OPERATING SYSTEM CHOICES}

Customer requirements for widely accepted, commodity-priced systems considerably constrain computer hardware and operating system choices. In practical terms, this implies some type of PCcompatible system running one of the Microsoft Windows operating systems. The Development Division takes the position that this is acceptable provided that

- An industrially hardened system is used,

- Failure of the system does not compromise safety,

- There are no real-time response requirements, and

- Windows NT is chosen.

An industrially hardened system was not an issue to DSO. The legacy LTTD system had no significant safety or real-time response issues, and none of the additional requirements change this. Windows NT was also not an issue for DSO. A rack mountable, industrially hardened PC system with Windows NT 4.0 Workstation was purchased for the first implementation of the ELTTD design. This system hardware is essentially the same as hardware chosen for the Intelligent Inspection System (IIS) design that the Y-12 Product Certification Organization employs.

A vast array of choices of data acquisition hardware and software exist for the PC/Windows platform. Development staff recommended National Instruments data acquisition hardware and support software (LabView or LabWindows), a choice that has been used successfully over the past several years and for which considerable expertise exists. LabView has gained considerable acceptance throughout the weapons complex, and Lawrence Livermore National Laboratory (LLNL) is one of the largest users of National Instruments products in the world. It is interesting to note that LLNL's laser cutting workstation, which DSO will ultimately own and operate, was designed primarily around National Instruments products.

\section{HARDWARE DESIGN}

A hardware design was created by use of a selection of National Instruments components to monitor and control the LTTD manifold valves, oven controls, cryopump, turbopump, and pressure and temperature readings. This design was placed in Y-12 Engineering records under the title "Enhanced LTTD Workstation" and may be retrieved by a request of Drawing Nos. E2E92042EA133 through E2E92042EA140. Appendix F presents the material from these drawings.

\section{COMPUTER SOFTWARE DESIGN}

This report will briefly describe the human interface and the top-level behavior of the ELTTD software design. Future technical reports will describe the internal features. 


\section{HUMAN INTERFACE DESIGN}

Figure 2 shows a typical front panel for the ELTTD design.

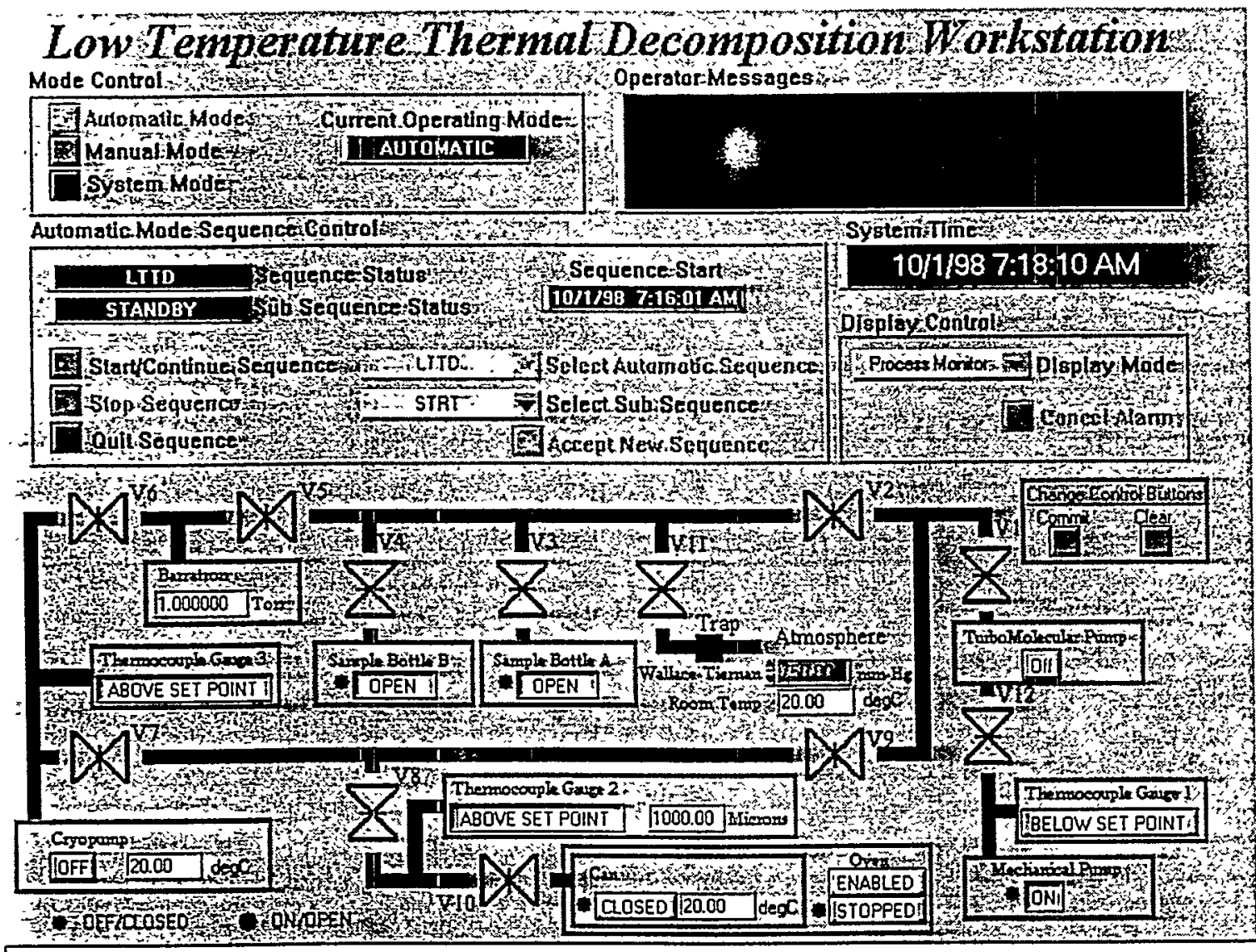

Fig. 2. Enhanced LTTD workstation sample front panel

The front panel is divided into a lower and an upper half.

The upper half contains controls and displays, which are always available. There include the ti $\because$, the system time display, mode control and status, automatic mode sequence control and status. larm indicator and alarm reset, and display control for the lower half.

The lower half contents depend on the current mode and on the operators' selection. The operator $y$ always select at least two panels-the operator message history and the trend display. System $\mathrm{n} \quad \mathrm{e}$ enables the selection of two more panels-one that allows password-protected access to system functions (shutdown) and one that shows internal system statistics. Manual mode enables the selection of a process monitor panel, which graphically depicts vacuum manifold status and allows manual control of the system. Automatic mode enables the selection of three additional panels-a 
process monitor panel (without the manual controls), an automatic sequence status display, and a process data display.

Figures 3-5 present additional examples of ELTTD front panels.

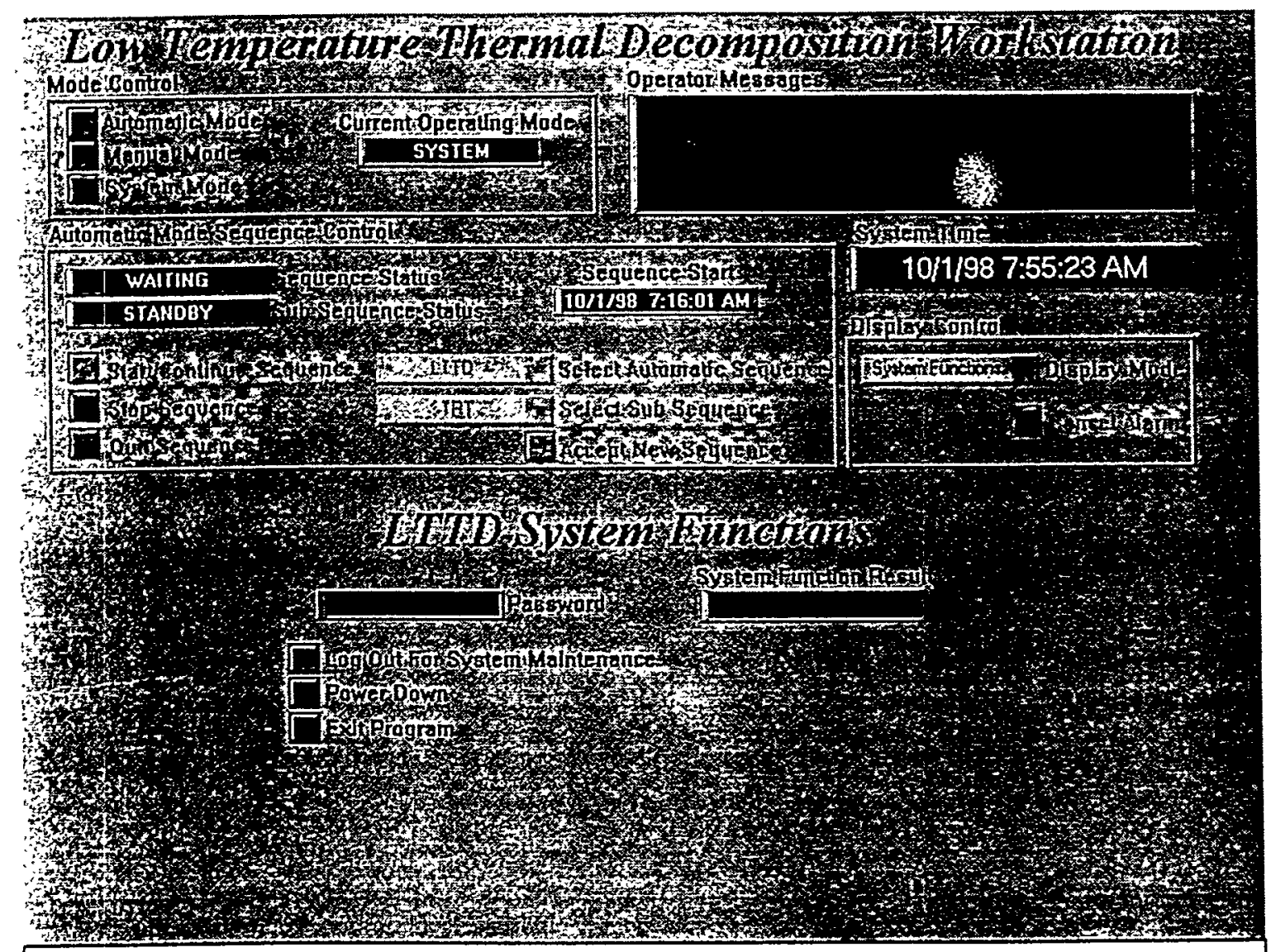

Fig. 3. System mode 


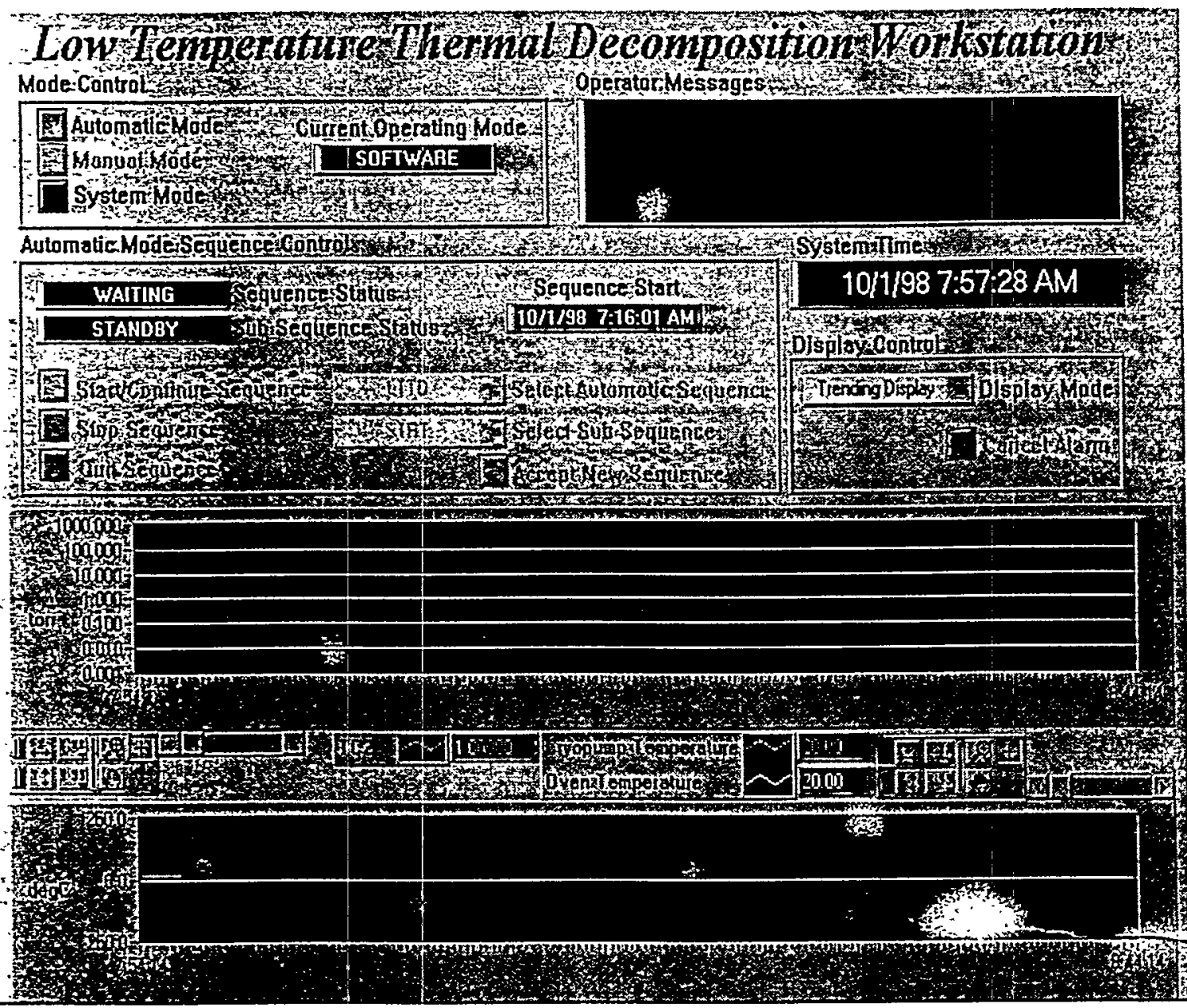

Fig. 4. Manual mode with trend display. 


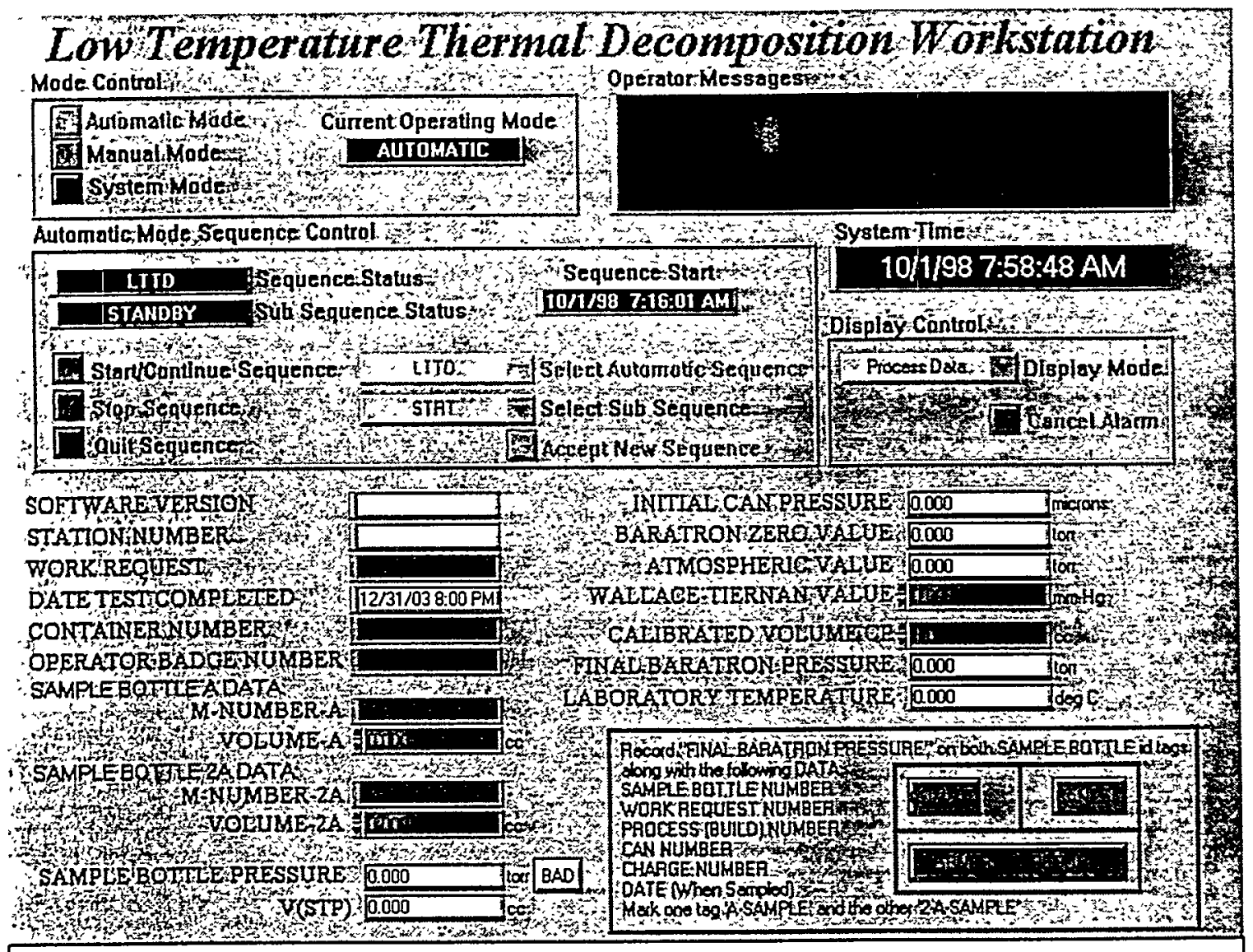

Fig. 5. Automatic mode process data/report generation 


\section{SUMMARY}

This report has detailed a new design for the controller and manifold for the Y-12 Plants' LTTD process. Future technical reports will provide internal detail of the software implementation and will describe extension of the design to support new LTTD measurement capabilities.

\section{ACKNOWLEDGEMENTS}

The author would like to acknowledge the contributions and support of DSO personnel, especially Dan Wilkins, Dale Conatser, Tom Fisher, and Randy Treece

The author would also like to acknowledge contributions from a number of Development personnel, especially Tom Morris, who was responsible for the enhanced LTTD manifold; Jim Basford and Jennings Cline, who provided guidance with regard to vacuum technology and other experimental issues; and Roy Pollard, who provided invaluable computer support. 


\section{APPENDIX A: INVENTORY OF LEGACY LTTD WORKSTATION}

DOCUMENTATION 


\section{Inventory of LTTD Legacy System Documentation (97/02/05)}

1. Source code for program "LTTD", 17-May-1989, AS-C-LTTD-1.3, runs under HP Basic Interpreter, $\sim 600$ lines, 16 pages

2. Source code for program "DECOMP", 17-May-1989, AS-C-DECO-1.3, runs under HP Basic, 600 lines, 14 pages

3. Procedure for LTTD Manifold Volume Calculation, 7 pages

4. LTTD, Manual Interface Photometal Panel, 4/15/88, I2E137698, CADAM drawing showing photometal panel assembly

5. LTTD, System Diagram/Assembly, 4/15/88, I2E143710, CADAM drawing showing system level connections

6. Maintenance Work Request to modify LTTD 012 (6A-406), 2/8/88, YX178210, 4 pages, instructions for installation of LTTD vacuum system controls, also MWR for LTTD 011 (6A-407) 4/20/88 and LTTD 013 (6A-408) 5/7/87

7. Flowchart for LTTD program, 9-Feb-88, 5 pages

8. Variable usage list for LTTD program, 4-Feb-88, 5 pages

9. Source code for program "VOLCALC", 3-Feb-1988, runs under HP Basic Interpreter, $\sim 600$ lines, 16 pages

10. Process time sequence, undated, 1 page

11. Materials list, undated, 1 page

12. Manual procedure for measuring LTTD manifold volume, undated, 3 pages

13. Hand sketches, undated, 5 pages

14. Data sheet on GE CR305/CR306 contactor, 2 pages 


\section{APPENDIX B: SOURCE CODE FOR LEGACY THERMAL}

DECOMPOSITION SOFTWARE 


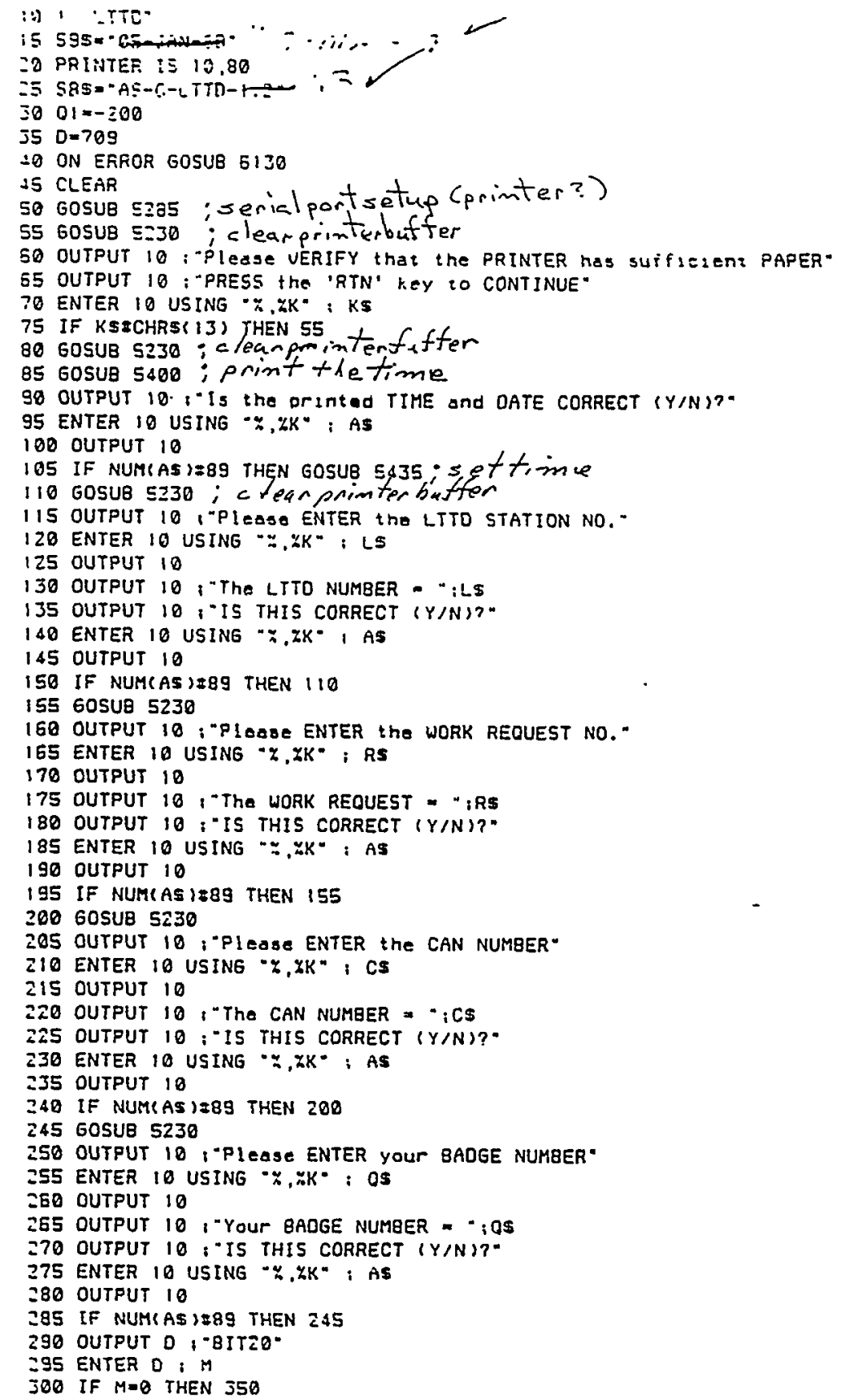




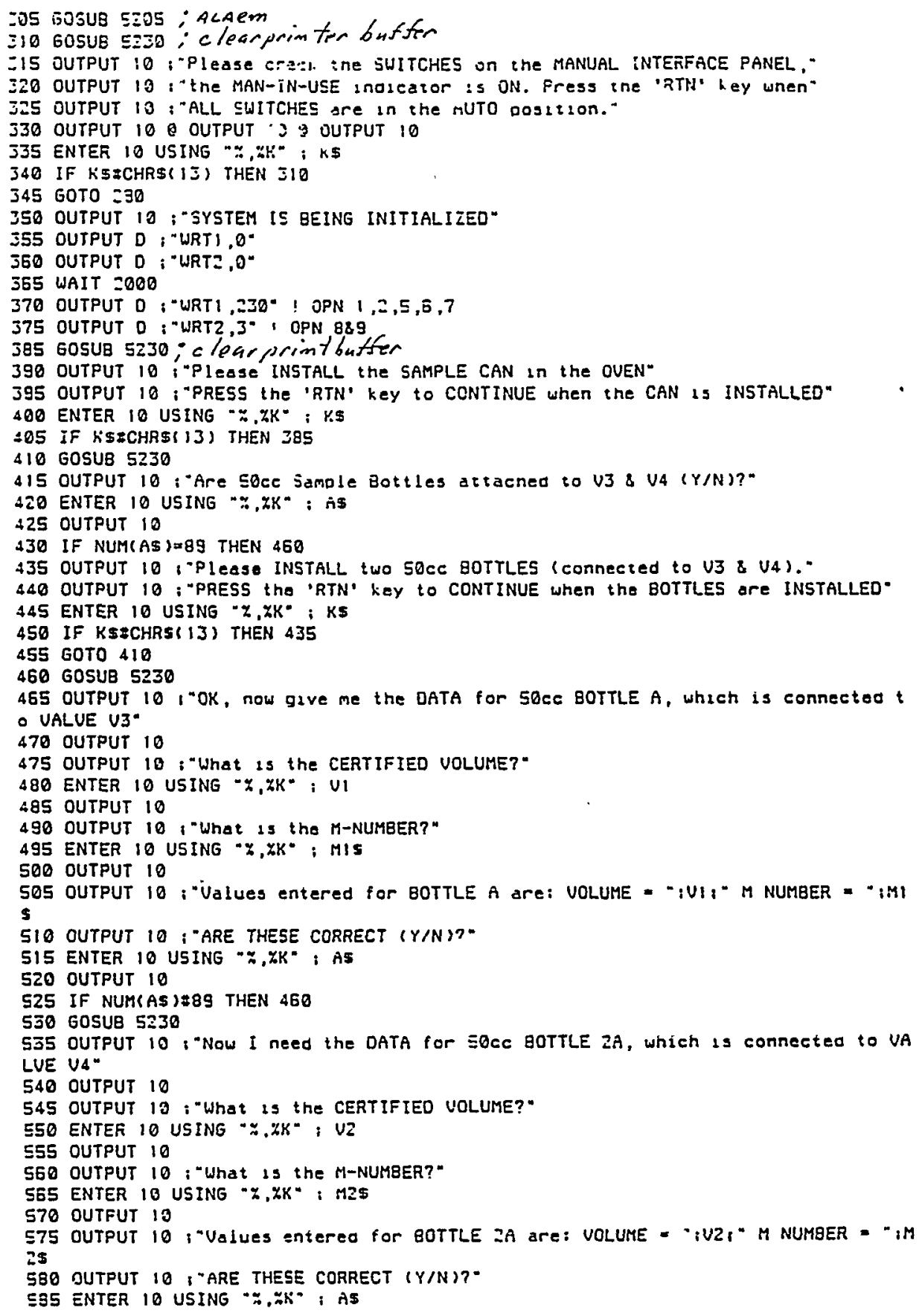

¿10 GOSUB 5250; Clearprim tre buffer

$=15$ OUTPUT 10 : Please cra: 1 ine SWITCHES on the MANUAL INTERFACE PANEL,

220 OUTPUT 13 : "the MAN-IN-USE inoleator is ON. Fress the 'RTH' hey unen

345 GOTO $=30$

IS BEING INITIALIZED

370 OUTPUT $0:-$ WRTI, $230^{\circ}$ ! OPN $1,2,5,5,7$

375 OUTPUT $0:$ "URT2,3" OPN 889

385 605U日 5230 ; clearprintbutier

400 ENTER 10 USING $\cdots, Z K^{*}:$ : KS

\$1 (13) THEN 385

GOSUB 5730

420 ENTER 10 USING * $\%$.

425 OUTPUT 10

430 IF NUM(AS) $=89$ THEN 460

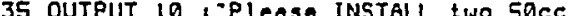

445 ENTER 10 USING $* Z, X^{*}$; KS

450 IF K\$:CHRS(13) THEN 435

455 GOTO 410

465 OUTPUT 10 "OK, now give me the DATA for 50ce BOTTLE $A$, which is connected t

- VALUE V3.

495 ENTER 10 USING * $\%, 4 K^{*}$; MIS

(15 ENTER 10 USING " $"$ "

540 OUTPUT 10

550 ENTEF 10 USING **.7K"; U2

555 OUTPUT 10

OUTFUT 19

580 OUTPUT 10 , "ARE THESE CORRECT (Y/N)?-

SS5 ENTER 10 USING “־, 


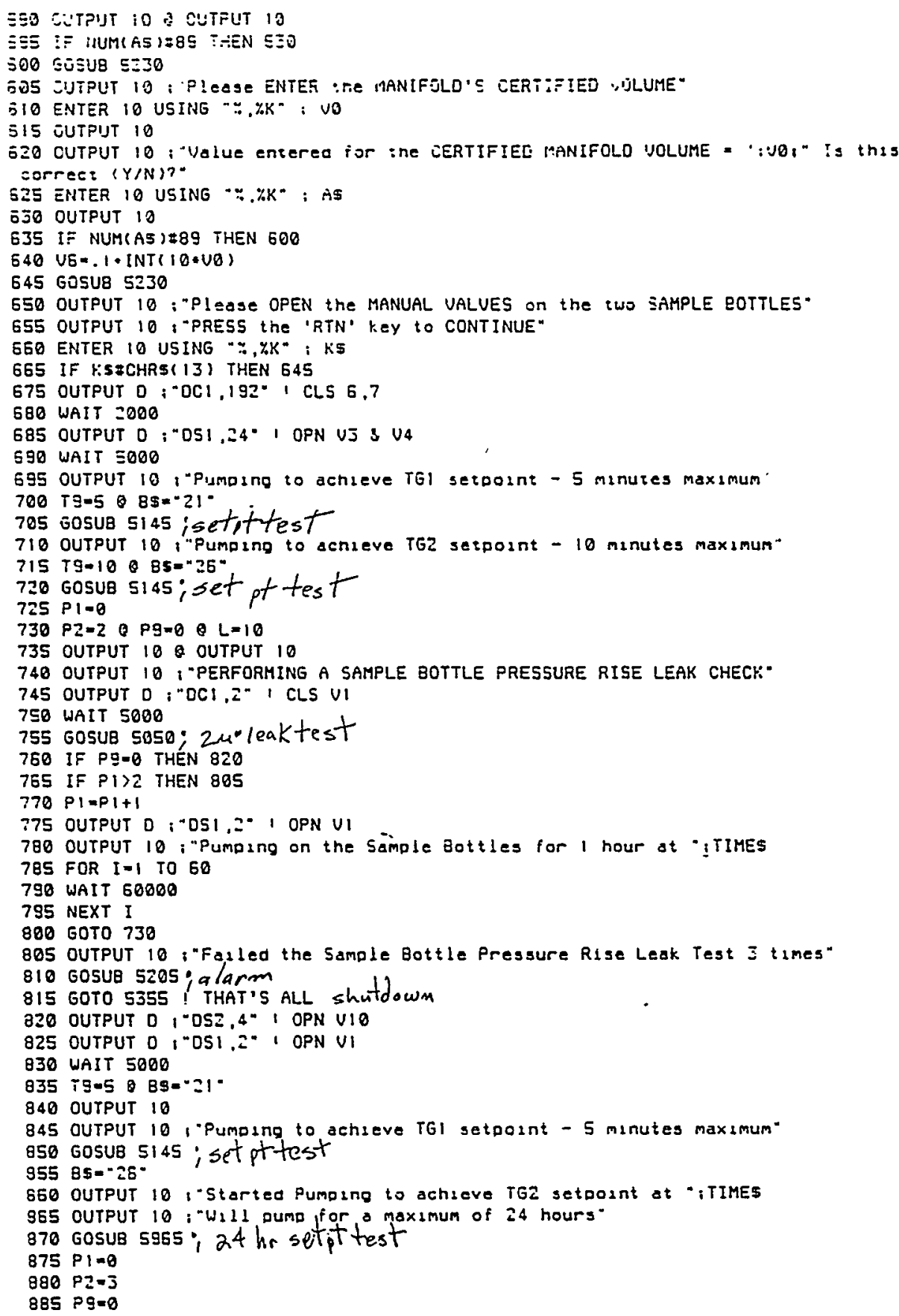




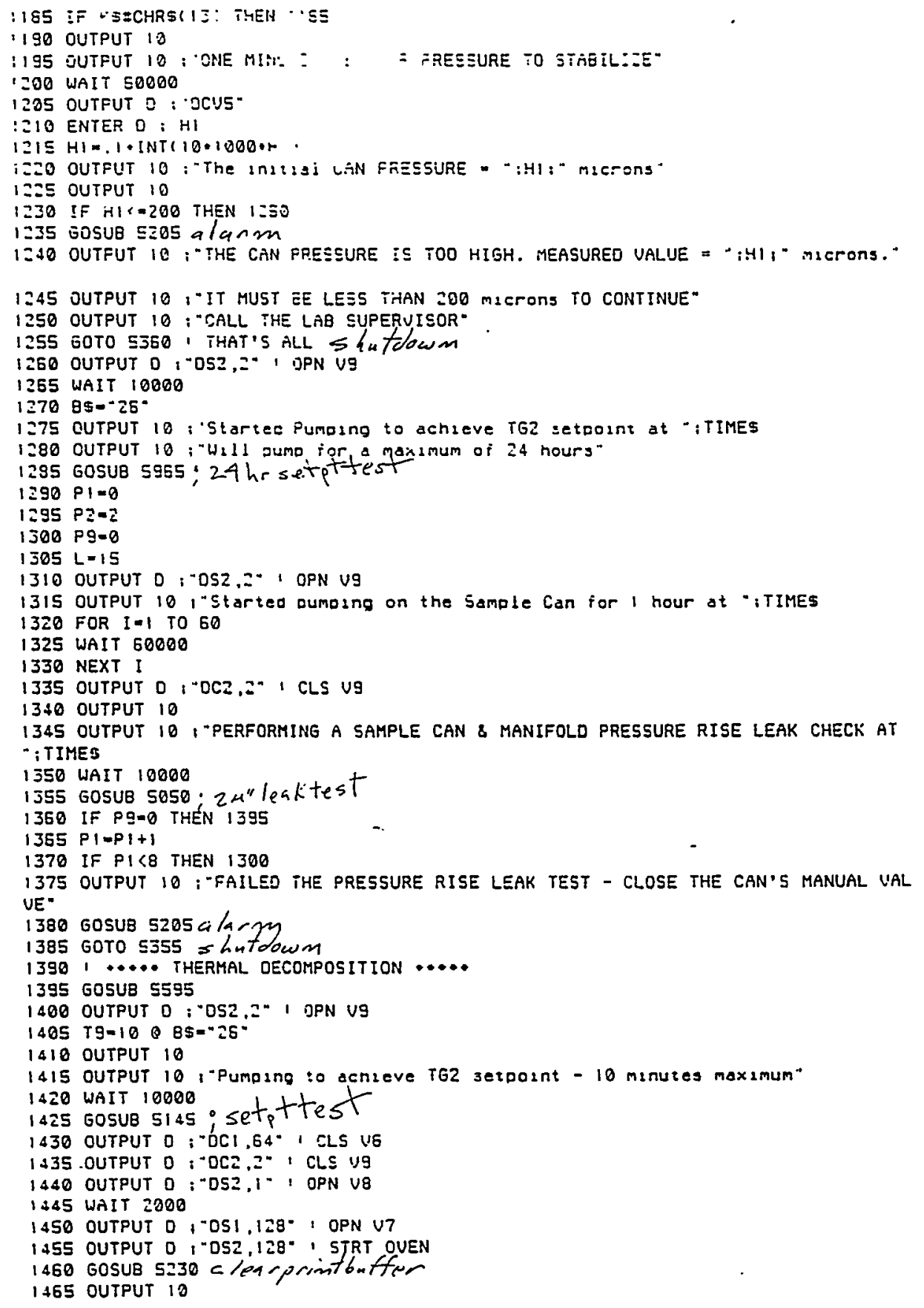




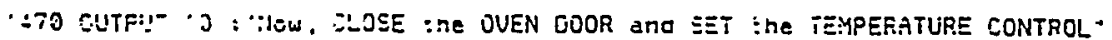

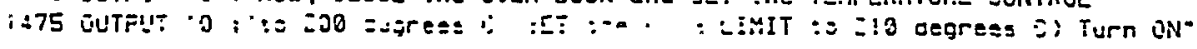

1480 UUTFU: $: 2$; the FOWER ENITCH ano RESET . :e HIGH LIMIt CONTROL."

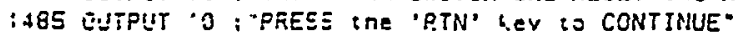

1490 CUTFUT $I J$ O OUTPUT $: 0$ O O OTF.jT $\cdot 3$

1495 ENTEP : : 3 USING $\because, \ldots 4^{*}: \therefore 5$

1500 IF $1: 5$ ECHRS 1 II: THEN 1460

1505 DUTFIJT 100 GUTPUT 100 OUTPUT 10

1510 OUTFUT :O : -ETARTING THERMAL DECOMPGSITION TEST AT*

1515 G0SUE $\equiv 400$ primt the fime.

1520 SO-TIME/JEÓO START TIME - HOURS

1525 Dos-ŨATES

1530 DOS-0OS[4,8 $13 * 119 * 8 D O S[1,2\}$ I START DATE

1535 OUTPUT 10 OUTPUT 10

1540 F $1=0$ Q 29:0

1545 GOSUB 5800 setiprint teemp ralues

1550 ON KE'? 1 GOSUE 1650

IS55 FOR $1=1$ TO

1557 FOR $\mathrm{H}:=1$ TO 50

!559 WAIT $\$ 0000$

1561 NEXT $r$

1565 IF $: 4$ THEN 1600

1565 IF F1:24 THEN 1600

1570 OUTPUT $0 ;$ : DCUS" $^{\circ}$

IS75 ENTER D; G9

1577 G9-.1.INT(10.1000.69)

1580 IF G\$129/2 THEN 1600

1590 OUTPUT 10 ; "Sample Can PRESSURE 15 " :69," microns at "itimes

1595 IF G9>29 THEN 29-69

1600 NEXT I

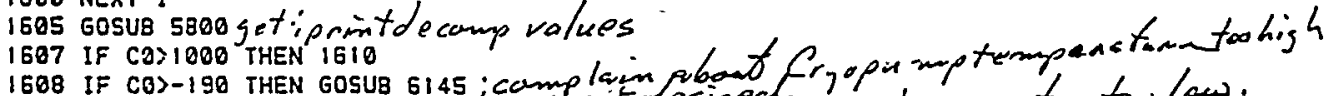

1610 IF FI<4 THEN 1640

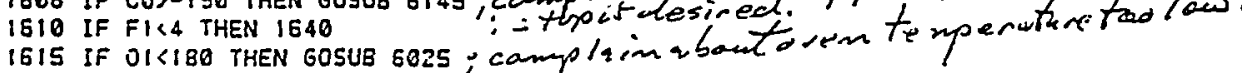

1620 TIS-IIMES

1625 T15=TISCI, 2$]$

1630 IF UALITISIS4 OR VALITY\$I: 14 THEN 1540

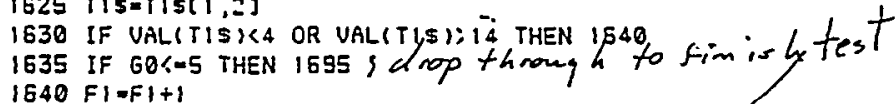

1645 GOTO 1550

1650 GOSUB 5900 get iprint decamp values

1655 sosub 5330 clearprimt ba ffer

1660 OUTPUT 10 : "Thermal Decompositign Test has been in progress for " $\mathrm{FI} \cdot 4 \mathrm{I}^{\text {" ho }}$ urs.

1665 OUTPUT $10,1^{-}$do you want to TERMINATE the test $(Y / N)^{\circ}$

1670 ENTEF 10 USING " $\because$, "艹K" : A As

1675 OUTPUT 10

$\therefore$

1680 IF NUM(AS) 289 THEN RETURN

1685 GOTO 1825

1695 UFF !:EY: $1<$

1700 OUTPIJT 10

1705 . OUTFUT 10 ; "PERFORMING ì 7 MICRON IN 15 MINUTE PPESSURE RISE CHECY"

1710 OUTPUT 10 : "TO OETERMINE, IF TEST IS COMPLETE AT*

1715 eOSUI $\equiv 400$; print thetime

1720 OUTPUT 10

I7:5 OUTPUT 0 ; "OCZ,!* I CLS UB

1730 WAIT $\equiv 000$

I735 CUTPIIT 0 ; -OCUE- - READ TGZ 


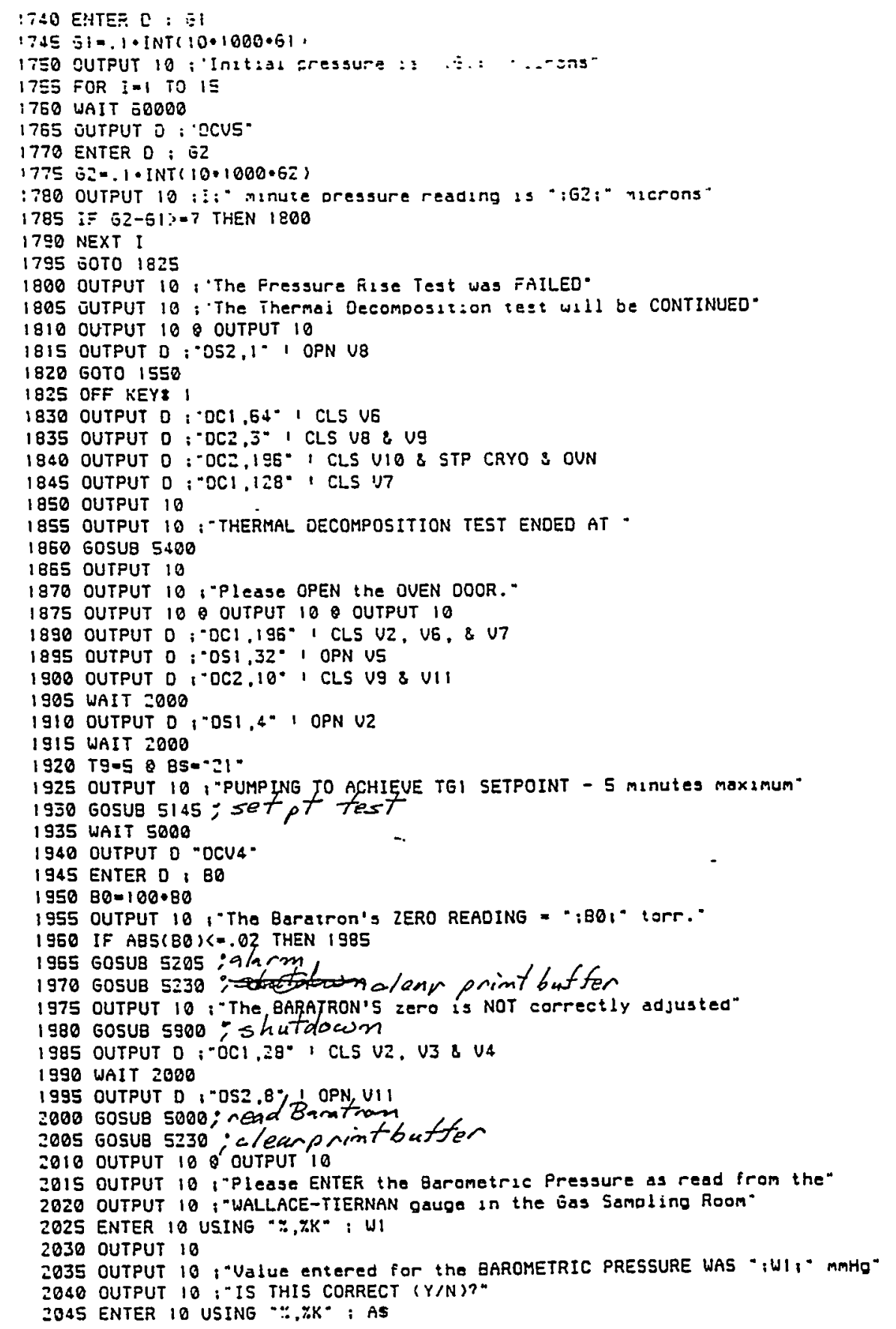


2050 DUTFUT: :0

20SE :F NUM(AS):89 THEN ::::

$\therefore 260$ GOSUR 5000

$206581=, 1 \cdot I N T(10 \cdot 100+R 2)$

2070 OUTPUT $10 ;$ "The Eara:ron's ATMOSPHERIC FRESSURE FEADING = "i81;" tarr."

:075 IF ABS(WI-BI\}-5 IHEN $=105$

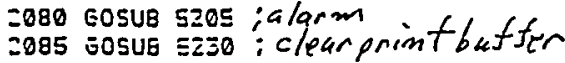

I090 UUTPliT 10 ; The difference in reading between the BARnitRoN and the"

zog5 UUTPUT 10 ; "WALLACE-TIERNAN gauge is greater inan the oilowable limit is to

re l.

2100 gosur $5900:$ shutdown

2105 OUTPUT D 1 "ÓCZ,9* I CLS UII

2110 WAIT $\equiv 200$

2115 OUTPUT $D:$ :DS1, $4^{*} 1$ OPN VZ

2120 WAIT 3200

2125 T $9=5$ a $85=-21$.

2130 OUTPUT $10:-P U M P I N G$ TO ACHIEUE TGI SETPOINT - 5 manutes maximum

2135 GOSUB 5145; setpteh ecK

2140 OUTPUT $10 ; * 30$ SECOND WAIT AT ";TIMES

2145 WAIT $\Xi 3000$

2155 OUTPUT 10

2160 OUTPUT 10 ; Walting for the Cryopump to reach thernal equllibrium*

2165 OUTPUT $D ; * O C 1,188^{\circ}$ I CLS U2, V3, V4, US \& V7

2170 OUTPUT $0 ;{ }^{\circ O C 2,11^{\circ}}$ I CLS VA, Ug \& VII

2175 WAIT 2000

2180 OUTPUT $D ;$ "DSI, 64" 1 OPN VE

2185 OUTPUT $D$, "OCV4"

2190 ENTER D B 85

$219585-.01 \cdot I N T(100 \cdot 100 \cdot 85)$

2200 OUTPUT 10

Z205 OUTPUT D I"TEMG"

2210 ENTER $0 ; C I$

2215 OUTPUT $10 \%$ Baratron PRESSURE $="$ " $85 \%^{\circ}$ torr

2220 IF C1:1000 THEN 2248

$2225 \mathrm{C} 1=.1 \cdot 1 \mathrm{NT}\left(10 \cdot \mathrm{Cl}^{\prime}\right)$

2230 OUTPUT $108^{\circ}$ CRYOPUMP TEMP $={ }^{\circ} \mathrm{iCl}: \mathrm{i}^{-}$jegrees $\mathrm{C}^{-}$

2235 GOTO 2245

2240 OUTPUT $10: " \quad$ CRYOPUMP TEMPP < -200 degrees $C \cdot$

2245 OUTPUT 10 1" AT " ITIMES

2250 OUTPUT 10 ; "There w111 be a 30 MINUTE WAIT before the next sat of readings

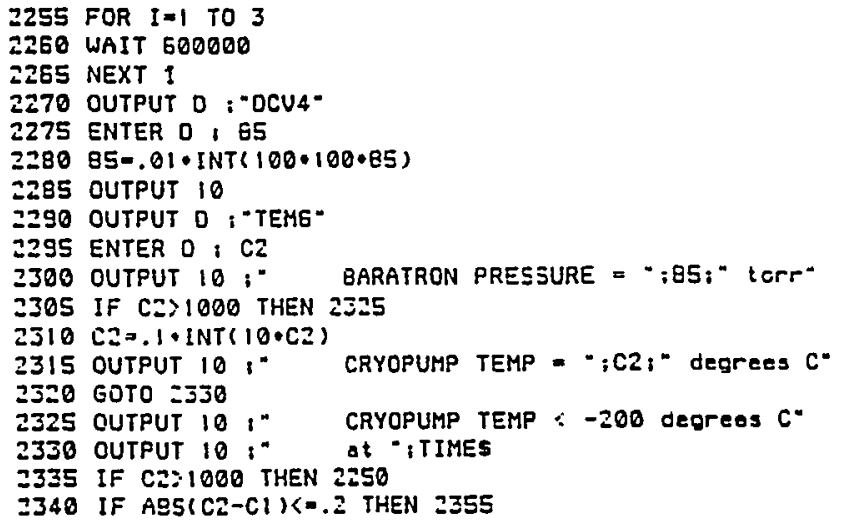


$: 3456: 1:: 2$

:ISO GOTE $=: \equiv 0$

ZISS OUTFUIT $0 ;-T E M 7-$

Z350 ENTER 0:

ZIES RO=. $\mathrm{I} \cdot$ INT(10-RO)

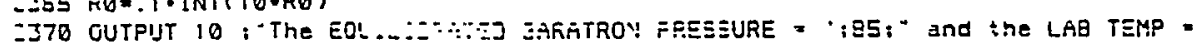

: $: 70$ Gosub 5230 oclearprint buffer

¿380 CUTPUT 10 :"ENSURE tnat the MANUAL VALUES on BOTH SDCC SAMPLE BOTTLES"

:335 OUTPUT $: 0$ i"are OFEN. =RESS the 'RTN' tey to CONTINUE"

ZZEO ENTER 10 USING " $\because, 2 K^{*}$; is

Z395 IF K.SICHRS(13) THEN $2 \equiv 75$

$2 \$ 00$ GUTPUT 10

Z\$05 OUTPUT 0 ; "DSI, 32 " I OPN US

2410 OUTPUT 10 ; "Valve US was OPENED;

$2420 \mathrm{BG}=.81+1 \mathrm{NT}(100+100 * \mathrm{RZ}$ :

2425 OUTPUT 10 ; "The BARATRON PRESSURE aiter CPENING US $=": B 6 ; "$ : orr."

2430 OUTPUT 10

2435 OUTPITT O:-DS1.8* 1 OPN U3



$245087-.01 \cdot 1 \mathrm{IN}(100 \cdot 1,00 \cdot R 2)$

\$55 OUTPUT $10 ;$;The BARATRON PRESSURE a ter OPENING UZ $=" ; 877^{\circ}$ : orr."

2460 IF ABS( $(B 6-87$ / $/ 86))=.015$ THEN 2485

2465 G0SUB $5205 ;$; 9 lam

2470 OUTPUT $10 ;$ THE CHANGE IN PREsSURE AFTER OPENING Uz WAS LESS THAN 1.5 PERCE NT"

2475 GOSUB 5230 ; clear printbutfer

2480 GOSUB 5900 : shutcown

2485 OUTPUT 10

2490 OUTPUT $0:-051,16^{\circ} 1$ OPN V4

2495 OUTPUT 10 : "Valve V4 was OPENED"

250060549500 ; wai, Banatrantobe stable: realit

$2585 B 8=.01 \cdot 1 \mathrm{NT}(100 \cdot 100 \cdot \mathrm{R2})$

2510 OUTPUT $10 ;$ "The BARATRON PRESSURE after OPENING VA $=: 88$, "

:515 IF ABS( $(87-98) / B 7))=.015$ THEN 2540

2520 GOSUB 5205 ; alarm

525 OUTPUT $10 ;$ THE CHANGE IN P̈RESSURE AFTER OPENING V4 WAS LESS THAN 1.5 PERCE

2530 GOSUB 5230 ; cleuchriat o s fem

2535 GOSUB 5900 ; shutdown

2540 OUTPUT 10 OUTPUT 10

2545 OUTPUT 10 ; The FINAL BARATRON PRESSURE (after opening BOTH U3 \& U4) - :B8 1 torr. -

2550 OUTPUT 10 ; "RECORD this value on both SAMPLE BOTTLE doontifi:sation tags"

2S55 OUTPUT 10 ; alono with the following DATA:"

2560 OUTPUT $10 ; "$ SAMPLE BOTTLE NUMBER"

2565 OUTPUT 10, " WORK REOUEST NUMBER"

2570 OUTPUT $10 ;$; PROCESS (BUILD) NUMBER"

Z575 OUTPUT 10 i $^{*}$ CAN NUMEEF"

2580 GUTPUT $10: "$ CHARGE MUMBER*

¿585 OUTPUT $10: "$ DATE (When SAMDled)"

ISSO CUTPUT 10 : "Mark ono $t 30$ 'A-SAMPLE' and tho other 'I-f-EPMPLE'-

2595 OUTPUT $10 \otimes$ OUTPUT 100 OUTPUT 10

2500 OUTPUT $0:-0 C 1.215^{\circ}$ ! CLS U3, V4, UE \& U7

2505 GOSUB 5230

2610 OUTPUT $10 ;$; 1 lease CLOSE the MANUAL VALUES on 8OTH SAMPLE BOTTLES:

2615 OUTPUT 10 , -PRESS the 'RTN' hey to CONTINUE whan the valvas are CLOSED'

2620 ENTER 10 USING " ". ZK" ; KS 
ZJZE IF TSZCHRS(1Z) THEM Z505

:בZO OUTPUT 10

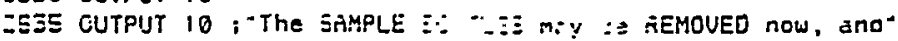

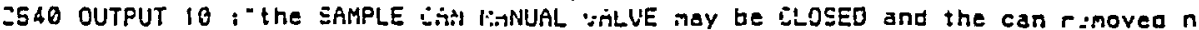

ou."

Z645 OUTFUT $0:{ }^{\circ}$ OCZ, $4^{-}$ZLS :.

2650 FOP. $\quad ?=1$ TO 18

ZSSS OUTPUT 10

Z660 NEXT I

2565 . .... CALCULATIONS .....

26701 PAra 6.7

2675 EI $=\overline{65} / 760 \cdot(273 /(R \theta+273))+1 / 10$

$2680 \mathrm{UH}=$

$2685 \mathrm{EI}-.01 \cdot$ INT $(100 . E 1$

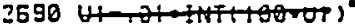

2695 OUTPUT $10 . ; "$....+FOLD HERE ......... FIJLD HERE ....... FOLD HERE

•*.*

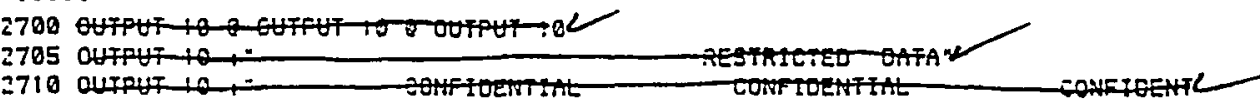

IAL"

2715 FOR $I=1$ TO $511^{2}$

2720 DUTPUT 10

2725 NEXT I

2730 OUTPUT $10:-$

2735 OUTPUT $10 ;:$

2740 OUTPUT $10: "$

2745 OUTPUT $10:-$

2750 OUTPUT $10:-$

2755 OUTPUT 10 :-

27E0 OUTPUT 10

2765 OUTPUT 10 :

2770 OUTPUT 10 :"

2775 OUTPUT $10:-$

2780 DUTPUT $10 \%$

2785 OUTPUT $10:-$

3790 OUTPUT 10 1-

2795 OUTPUT $10:-$

2880 OUTPUT 10

ZQOS OUTPUT 10 :"

2810 OUTPUT $10:$

2815 OUTPUT $10 \%$

2820 OUTPUT $10:-$

Offoctintiat

CONFIOEATA

2825 FOR $\quad I=1$ TO 5

2830 OUTPUT 10

2835 NEXT I

2840. OUTPUT $10 ;$;

2845 OUTPUT 10 :"

2850 OUTPUT $10:-$

2855 FOR I $=1$ TO 4

2860 OUTPUT 10

2865 NEXT I

2870 OUTPUT 101 -

2875 OUTPUT 10

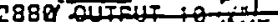

2885 JUTPUT TO OUTPUT 1

2890 OUTPUT 10

2895 OUTPUT 10

2900 OUTPUT 10 :"

SOFTWARE UERSION ":S85:" "

STATION NUMBER - :LS

WORK REQUEST - ;RS

DATE TEST COMPLETED *:DS

CONTAINER NUMBER $\cdot$; CS

OPERATOR BADGE NUMBER ":OS

SAMPLE BOTTLE A DATA:-

M-NUMBER = - IMIS

VOLUME - ":VI

SAMPLE BOTTLE ZA DATA:-

M-NUMBER $=\because$ iMZS

VOLUME $=\cdot i$ VZ

SAMPLE EOTTLE PRESSURE $=" i 881^{\circ}$ torr"

THE INITIAL CAN PRESSURE WAS " $\mathrm{HI}:$ " mierons"

THE BARATRON'S ZERO VALUE WAS " $: 30^{\circ}$ " tore"

THE BARATRON'S ATMOSPHERIC VALUE WAS * $1811^{*}$ "

THE WALLACE-TIERNAN BAROMETER UALUE WAS "; w1" mMHO

THE CALIBRATED VOLUME OF THE CRYOPUMP IS";UG: $:^{\circ} \mathrm{ce}$ "

THE FINAL BARATRON PRESSURE $=": B 5 i^{\prime \prime}$ torr"

THE LABORATORY TEMPERATURE * " $\mathrm{C} 2 ;$ "DEG C*

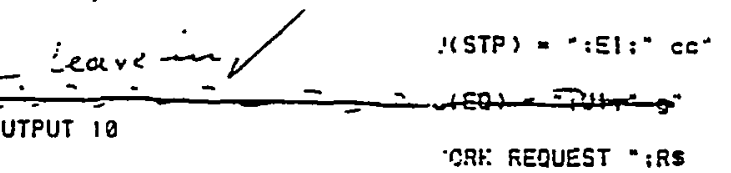

CRF: RERUEST * :RS

ONTAINER NUMEEF * :CS 


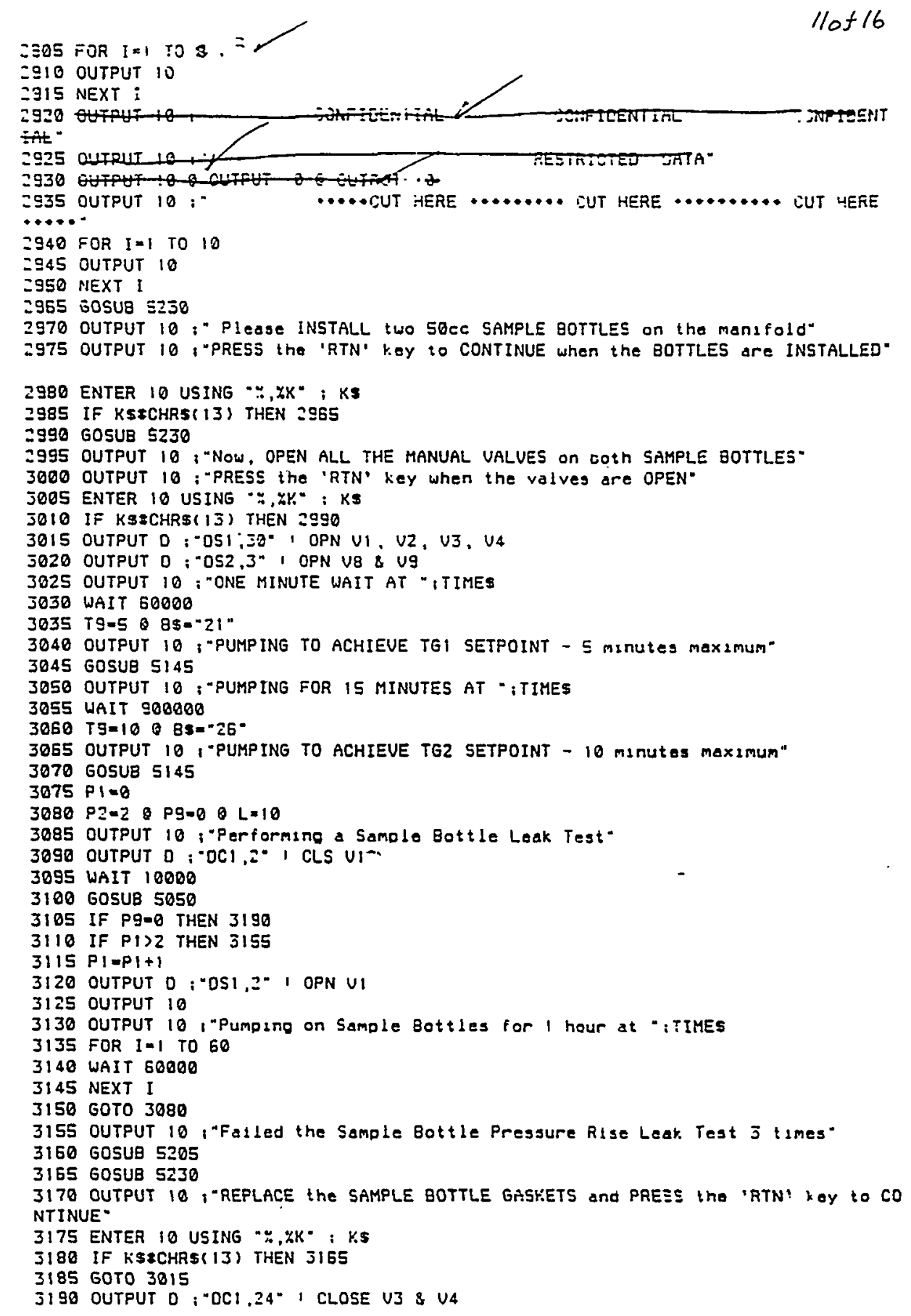


z! GE

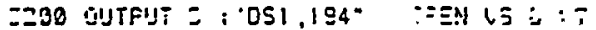

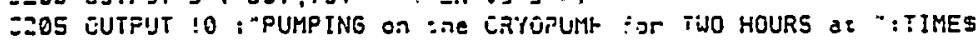

¿210 OUTPUT $: ;$ :WRTZ,Z*

$Z=15$ FOR $i=1$ TO 8

$3=20$ WAIT $\$ 20000$

:ב2S NEKT

ZZJO OUTPUT D : "OCUS"

I235 ENTEF. $0 ; 04$

$3240 \mathrm{G} 4=.1 \cdot$ !NT( $10 \cdot 1000 \cdot 64)$

3245 GUTPUT $: 0$ :"The CRYOPUMP' 9 FRESSURE IS " $: 641^{*}$ microns at "itIMES

3250 IF $64: 10$ THEN 3205

3255 OUTPUT 10 ; "PUMPING on the CRYOPUMP for ONE HOUR at "iTIMES

3260 FOR $i=1$ TO 4

3265 WAIT $\$ 80000$

3270 NEXT I

3275 OUTPUT O : "WRTI.,

3280 OUTPUT D I"WRTZ, $0^{\circ}$

3285 OUTPUT 0 : “WRTI, $38^{*}$

3290 OUTFITT D : "WRTE, $3^{\circ}$

I295 OUTPUT $10 ;$ :-SYSTEM HAS EEEN RETURNED TO THE STANDBY STATE-

3300 OUTPUT 10 : "PROGRAM CONTROL HAS GEEN TERMINATED AT *

$\Xi 385$ GOSUB 5400

$\$ 310$ OUTPUT $10 \Leftrightarrow$ OUTPUT $10 \Leftrightarrow$ OUTPUT $10 \$$ OUTPUT 100 OUTPUT 10

3315 STOP

5000 , ..... READ BARATRON .....

5005 OUTPUT 10 : "WAITING FOR BARATRON READING TO STABILIZE"

5010 OUTPUT D : "DCU4"

5015 ENTER D R RI

5020 WAIT 2000

5025 OUTPUT $0,-$ OCUA-

5030 ENTER D : R2

5035 IF ABS(R2-R1)<.0001 THEN RETURN

5840 RI-R2

5045 GOTO 5020

5050 I .... 2U LEAK TEST ....

5055 OUTPUT 0 ; "DCU5*

S060 ENTER D, G4

5055 G4= $1 \cdot 1 N T(10.1000 .64)$

5070 OUTPUT 10 :"Performsng a "iP2:" micron, "iLi" minute pressure rise leak tes

1.

5075 OUTPUT 10, "Initial pressure is " $i 64 t^{\text {"merons" }}$

5080 FOR $I=1$ TO L

5085 WAIT 50000

5090 OUTPUT $0,{ }^{-O O C U 5^{\circ}}$

SOSS ENTER D; GS

5100 G5-.1.INT(10.1000.65)

5105 OUTPUT $10, \mathrm{I}^{\circ}$ minute pressure reading $1 \mathrm{~s}^{\circ}: 65^{\prime \prime}$ mscrons"

5110 IF ABS(64-65)>P2 THEN 5135

$5 I I S$ NEXT ?

5120 RETURI

5125 PQ-1

S130 OUTPUT 10 : "FAILED THE" IL: " MINUTE PRESSURE RISE LEAK TEST"

5155 OUTPUT 10 \& OUTPUT 10

5140 RETURN

$5145, \ldots \ldots \ldots \ldots$ SETPOINT TEST *....

$5150 \mathrm{~N}=1$

SISS OS- EIT

5160 OUTPIJT $0: 05$

5165 ENTE: $0: 31$ 
$\vdots 770$ IF $\equiv:=1$ THEN FETIJPN

इi75 WritT 10000

$\equiv 180 \mathrm{~N}=\mathrm{N}+1$

SI8S IF NLE+TO THEN 5155

SI90 GUTPUT 10 ; "CANNOT FIMMP EELOW SETPOINT IN "; $T$;" MINUTES ALLOWED"

इIgS GOSUR 5305; Aharm

SID0 GOTO S3S5: shutdow m

5205 . .... ALARM .....

SI10 OUTPUT O I CLSIO. $^{-}$

SIIS WAIT 100

5Z20 OUTPUT D : "OPN10"

5225 RETURN

5230 , .... CLEAR TI'S BUFFER ....

5235 SET TIMEOUT $10 ; 1000$

5240 ON TIMEOUT 10 GOTO 5260

5245 ON EAROR GOSUB 5220

S250 ENTER 10 USING $": .8^{\circ} ; i$

5255 GOTO 5250

5260 SET TIMEOUT 10.0

5265 ON ERROR GOSUB 6130

S:70 RESET 10

5275 GOSUB 5285

5280 RETURN

$5285, \ldots . .$. SERIAL PORT SETUP .....

5290 CONTROL $10.4 ; 25$

5295 CONTROL $10.9 ; 139$

5300 CONTROL $10,11,194$

5305 CONTROL $10.12: 13$

5310 CONTROL 10,$14 ; 17$

5315 RETURN

5320 , .... SERIAL PORT ERROR .....

S325 SET TIMEOUT $10: 0$

5330 OFF ERROR

5335 I ERROR $=114$ IS BUFFER OUERRUN

5340 IF ERRN $=114$ THEN RETURN

5345 OUTPUT 10 ;-SERIAL PORT ERROR NUMBER ";ERRN, OCCURRED AT LINE * ERRL

5350 STOP

5355 1 .... SHUTOOWN .....

5350 OUTPUT O, "WRTI, $0^{\circ}$

365 OUTPUT $0, "$ WRT2 $0^{\circ}$

5370 OUTPUT $0 ;$ : WRTI, 38*

5375 OUTPUT D , -WRT2,3.

5380 OUTPUT 10 "PROGRAM CONTROL IS BEING TERMINATED-

5385 GOSUB 5400

5390 OUTPUT 10 OUTPUT 10 OUTPUT 10 OUTPUT 10

5395 STOP

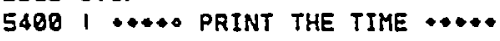

5405 D $15=0 A T E S$

5410 DS=01s[4,5]8" - 801\$[7,8]8" - "801\$[1,2]

5415 HS-TIMES

5420 TSOHS[1,5]

5425 OUTPUT $10: *$ TIME ":T\$:" DATE ";OS

5430 RETURN

5435 \& $\cdots$... SET TIME ROUTINE *.*.

5440 GOSUB 5230

5445 OUTPUT 10 ;-ENTER the DATE, format: MM,DO,YY, e.0., 04,06.83 for Hor 116,1 $983^{\circ}$

5450 ENTER 10 USING *.,ZK"; $2 \$$

5455 OUTPUT 10

$5460 \mathrm{Ms}=i \$(1,2)$ 


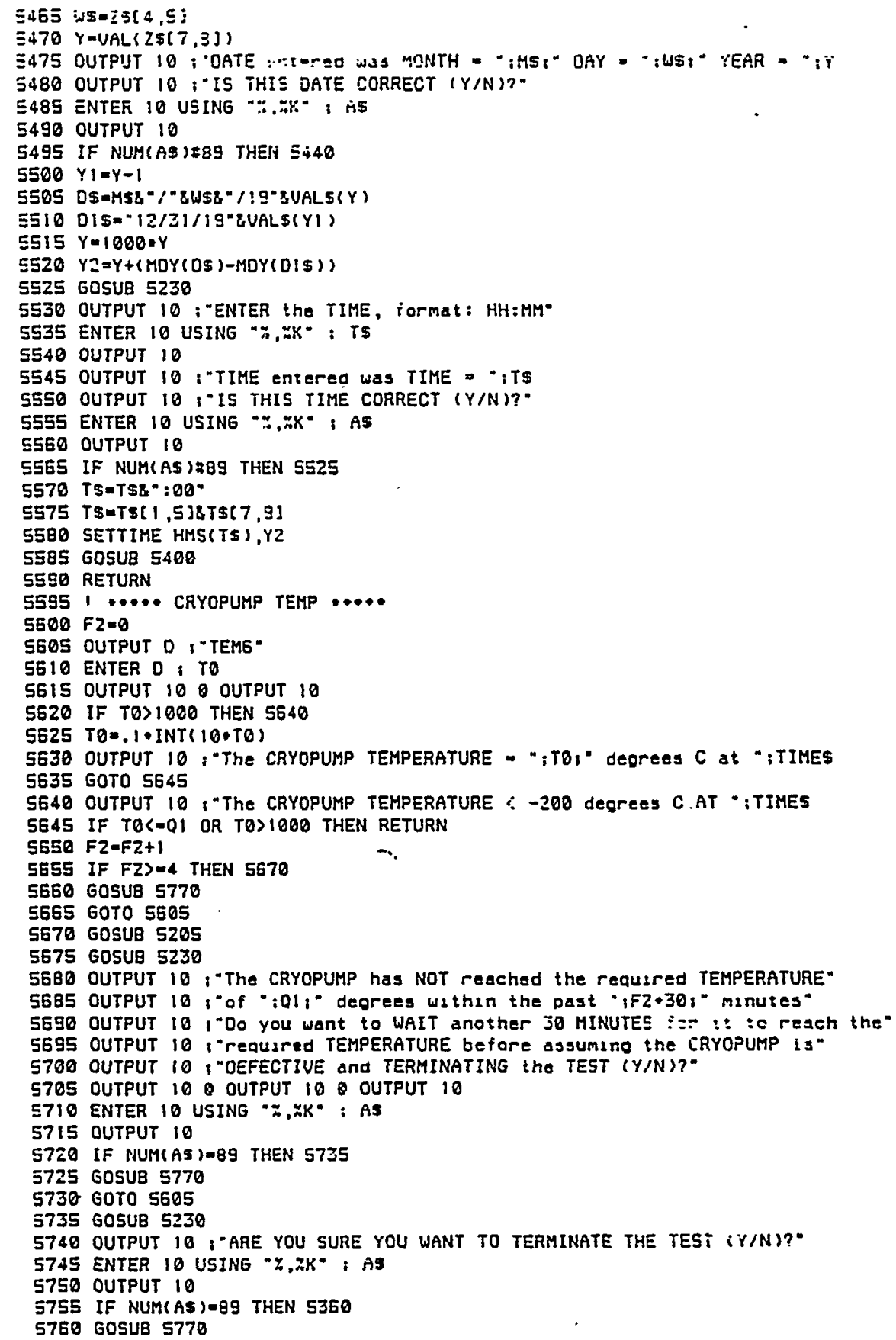




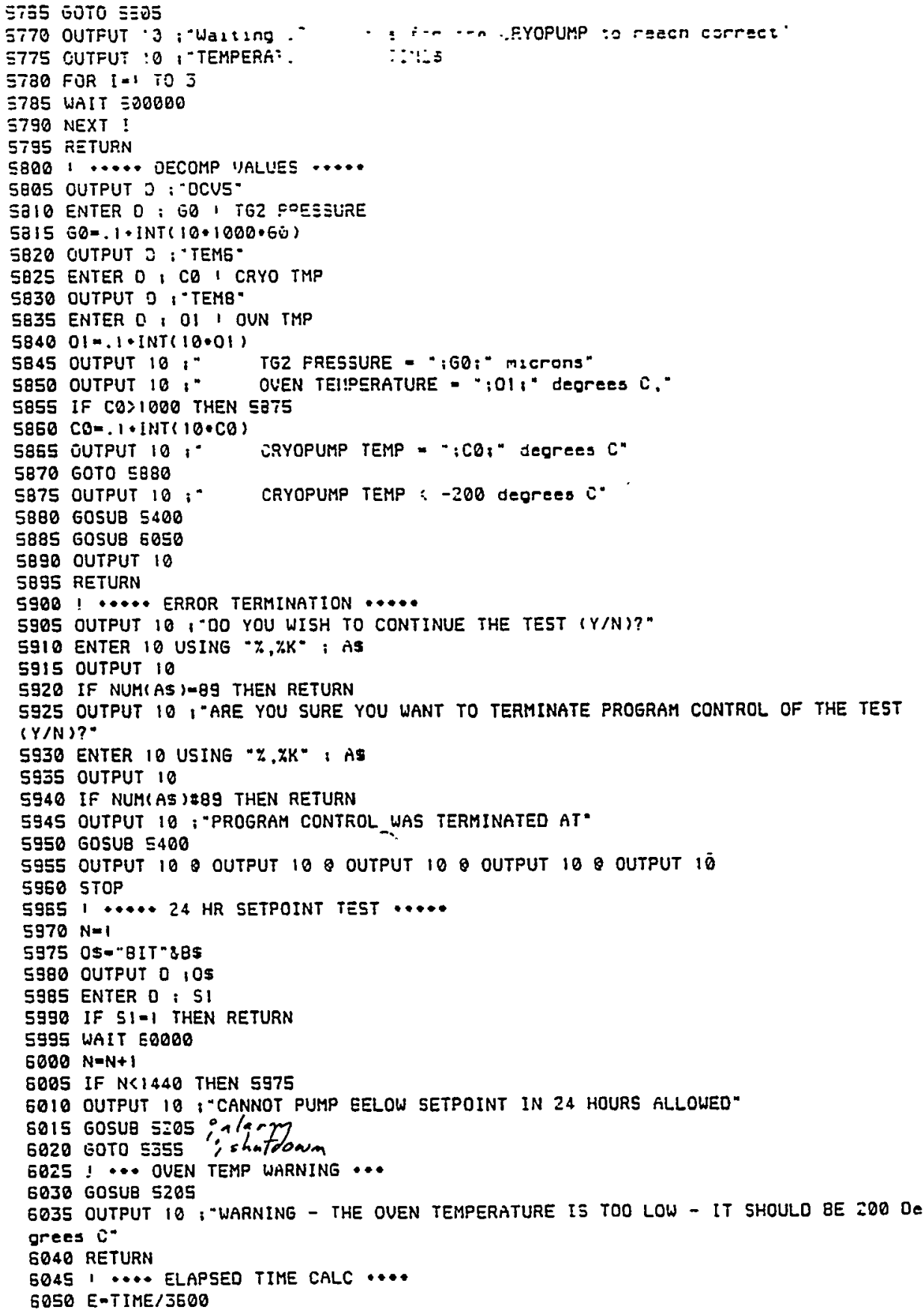




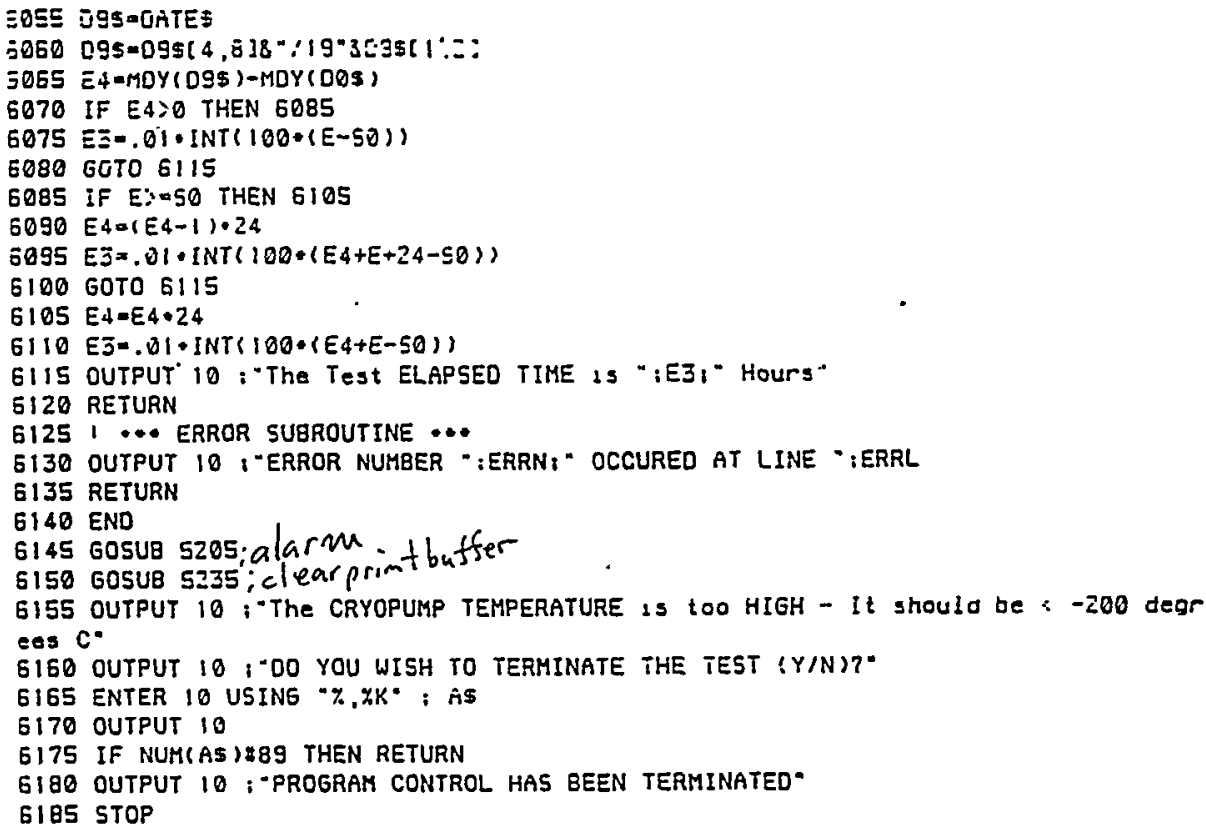


APPENDIX C: FLOWCHART FOR LEGACY THERMAL DECOMPOSITION SOFTWARE 

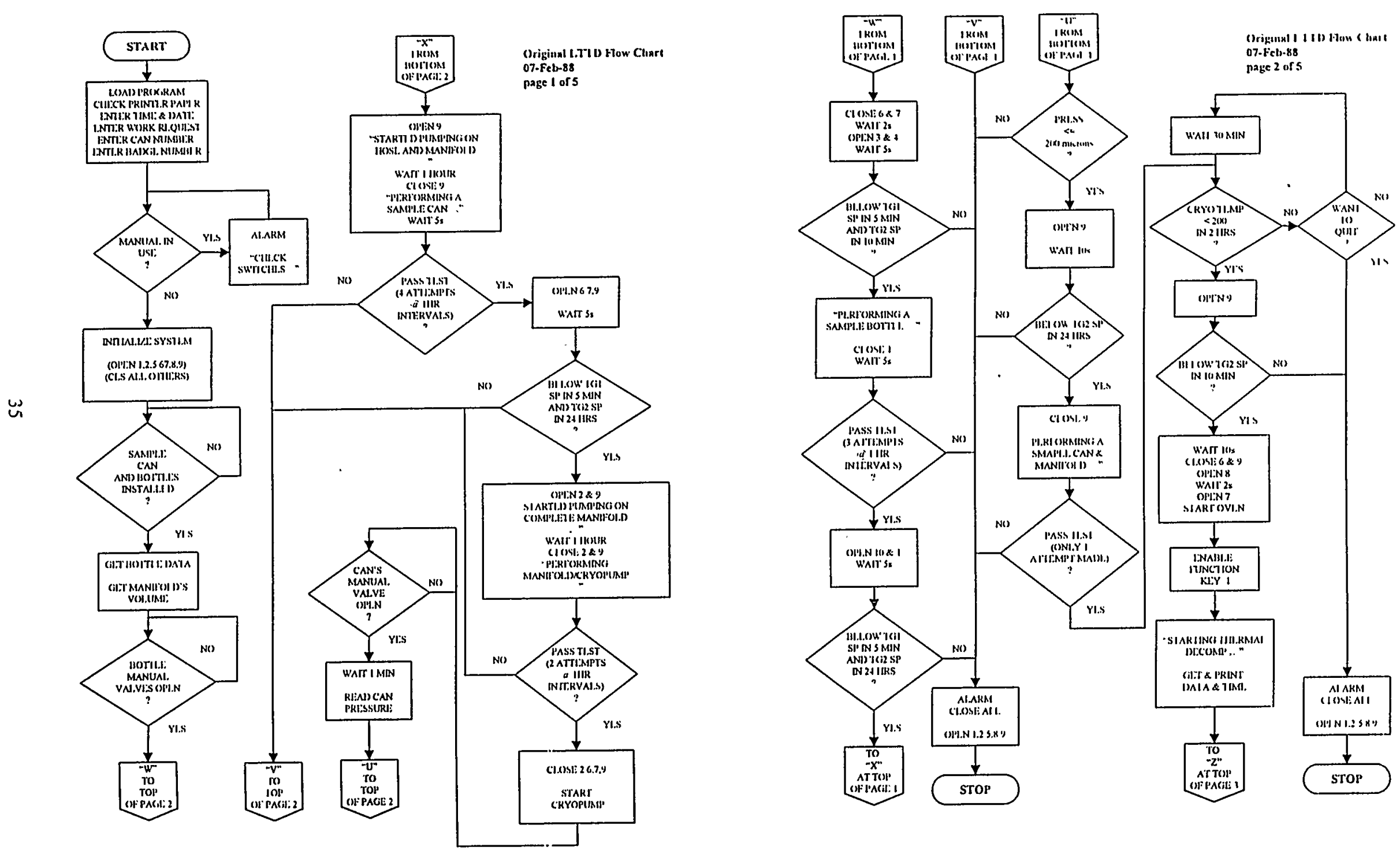

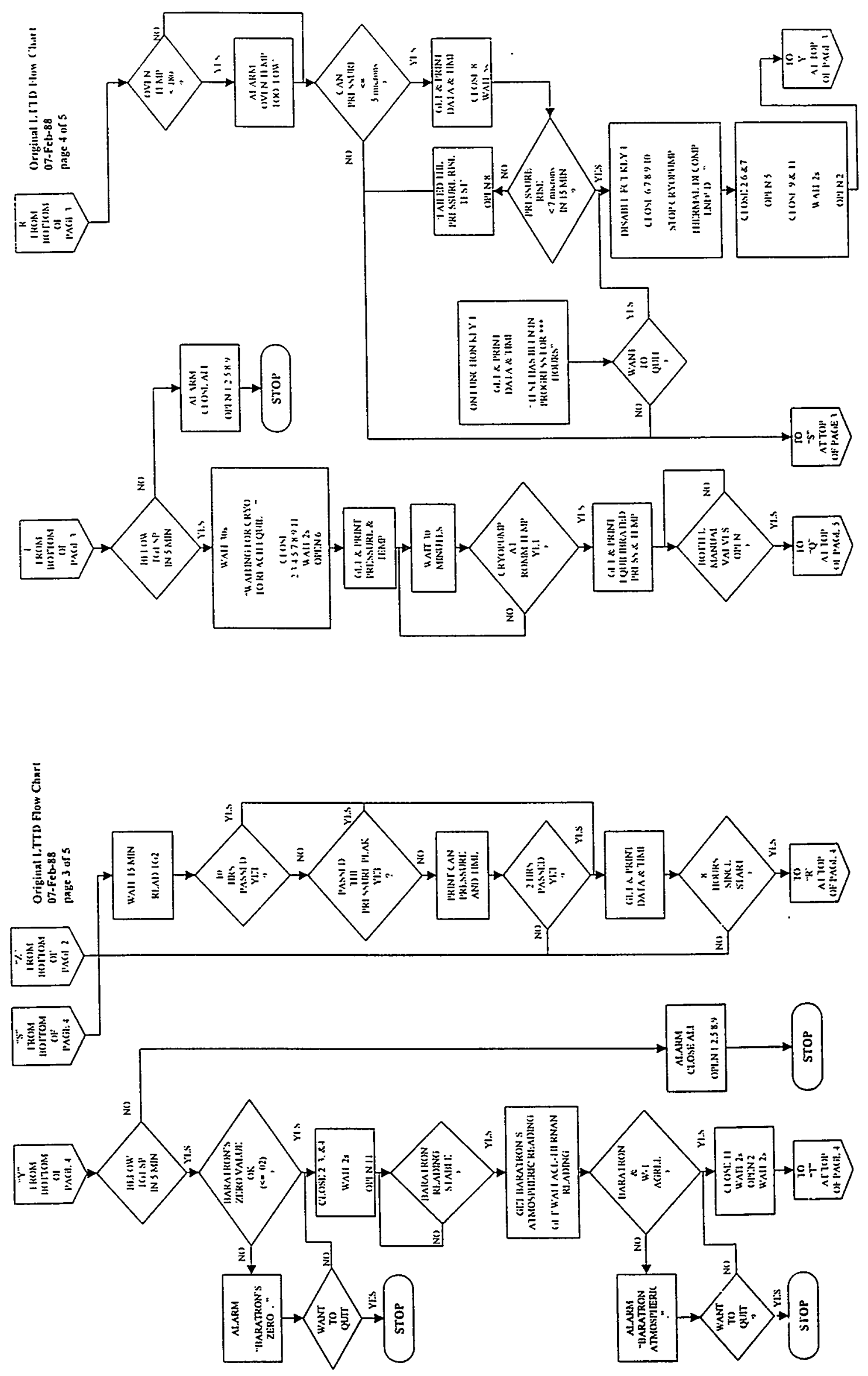


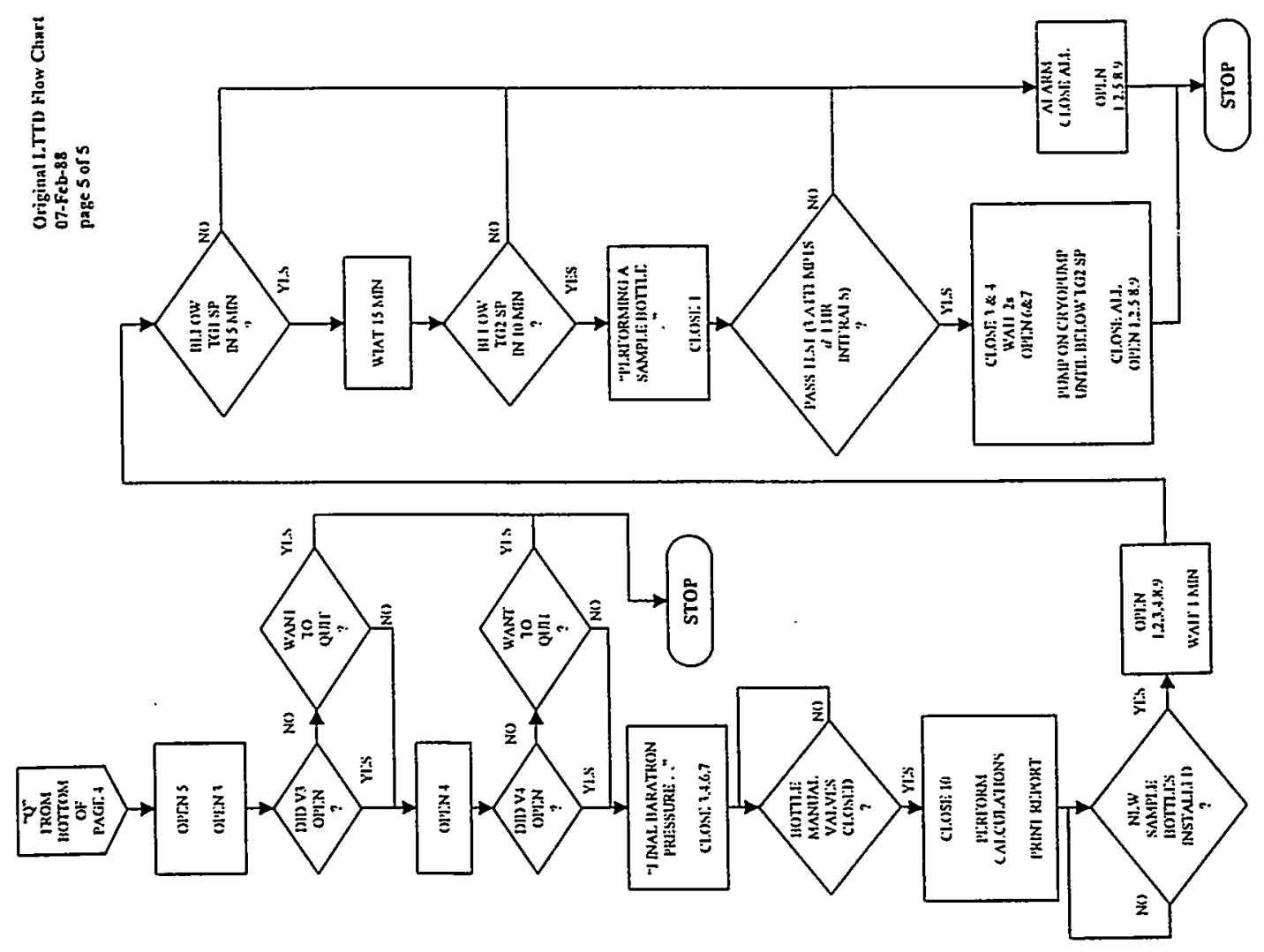


APPENDIX D: SOURCE CODE FOR LEGACY MANIFOLD VOLUME CALIBRATION SOFTWARE 
50 1 THIS FROGKAM IS UTILIZED TO COMPIJTE THE LTTO'S MANIFOLD VOLUME

$\therefore 9$

501 S $25-\bar{z}=-30$

50 PRINTER IS 10,00

$70 \quad D=709$

aO CLETRR

S0 GOSUB 4950 I INITIALIZE THE BEETAL PORT

100 OUTPUT $D ;-8 I T 20^{\circ}$ I TEST MAN IN HSE

110 ENTER D; $M$

120 IF $M=0$ THEN $Z 200$

130 GOSUB 4790 ! START FLARM

140 GOSUE 4640 I GLR TI'S ZUF



160 OUTPUT $10 ;$;

170 OUTPUT 10 i"ALL SWITCHES are in :he AUTO position."

180 OUTPUT 100 OUTPUT 10 OUTFUT is

190 ENTEP. 10 USING "\%,\%" : KS

200 IF KSICHRS (IS) THEN 140

210 GOTO 100

220 OUTFUT $10: "$ :EYSTEM IS EEING INIT:ALIZEO"

730 OUTPUT 0 ; “WRTI 0 "

240 OUTPUT 0 : "WRTE, O"

250 OUTPUT Õ : "WRTI, 33

260 OUTPUT $C ;$; WRTZ, $3-$

270 OUTPUT 10 ;"PUMPING TO ACHIEUE :51 EETPOINT*

$280 \mathrm{ES}=-21$.

290 GOSUB 4670 \& BELOW TGI SP?

300 OUTPUT 10 ; "PUMPING TO ACHIEUE TEZ SETPOINT"

$31085=-26 "$

320 GOSUR 4670 , EELOW TGZ SP?

İO GOSUB $\$ 840$, CLR II'S BUFF

340 OUTPUT 10 ;"Please IHSTALL t

350 OUTPUT 10 ;"sample oottles. FREEE $t h=$ 'STTN" tey is CONTINUE"

J60 ENTER 10 USING " $\%, \because K "$; KS

370 IF T:S\$CHRS(13) THEN 330

380 OUTPUT 10 :-ARE THE SO0CC BOTTLE INSTALLED (Y/N)-

390 ENTEF 10 USING " $\#, \because K^{\prime \prime}$; AS

400 OUTPUT 10

410 IF NUMIAS IZ89 THEN 330

420 GOSUB 4840 I CLR TI'S BUFF

430 OUTPUT 10 , "OPEN the MANUAL VALLE: 3 ) on the 500ce gottles"

440 OUTPUT 10 ;"PRESS the 'RTN' l.ey : 0 CONTINUE'

450 ENTER 10 USING “ $\because$,

460 IF KSaCHRS(13) THEN 420

470 OUTPUT $0 ;-051,24^{*}$ I OPN UJ, 134

480 GOSUB 4840 I CLR TI'S BUFF

490 OUTPUT 10 ; ${ }^{-G}$ Give me the DATA for $500 \mathrm{cc}$ BOTTLE $f$, Jnzch is connected to VALVE ט3"

5DO OUTPUT 10

510 OUTPUT 10 ; "Whas is the CERTIFIE? UOLUME?-

520 ENTER 10 USING “" * "

530 OUTPUT 12

540 OUTPLIT 10 ; What is the M-NUMBEs?-

\$50 ENTEF 10 USING " $\% . \% K^{\prime \prime}$; MIS

560 OUTPITT 10

570 OUTPUT 10 ; "Values entered for EOTTLE A are: VOLUME = "IVI:" M NUMBER = "iM!

5

5B0 OUTPUT 10 


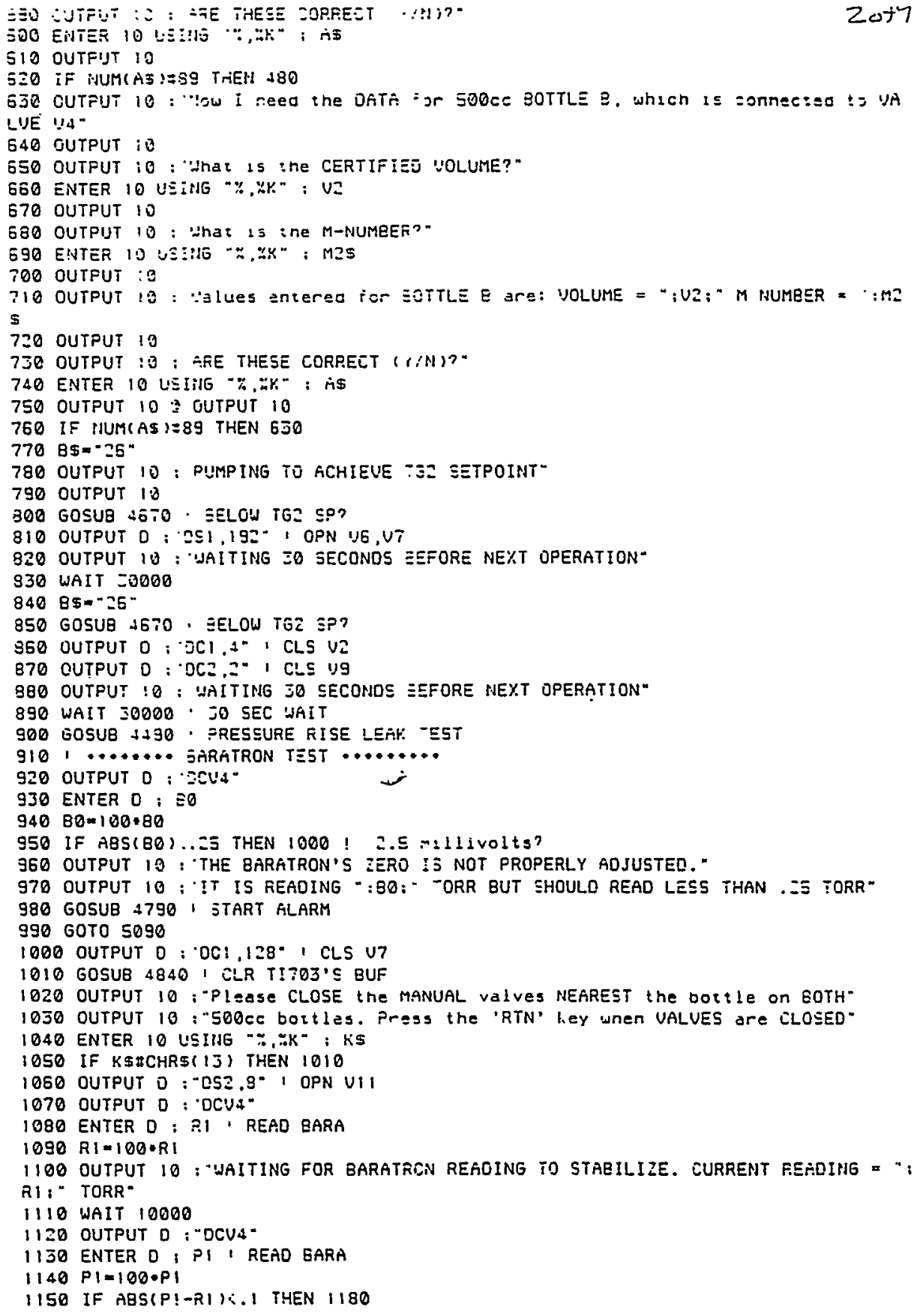


-

1170 GOTO 1100

1180 OUTPUT 10 :"THE FI:!AL EARATAON FEATOING = "

IISO GOSUB 4840 . CLEAR Ti'ミ SUF

1300 OUTPUT 10 :"Flease ENIEF :he Earcmetras Gressure es raad from ine"

1210 OUTPUT 10 : "NALLACE-i!ERNAM gage in the Sas Samci:ng Room"

IIZO EITTER 10 UEIHG * * : : :

II:E GUTPIST 10

1340 OUTPUT 10 : Value entereo for :he EAROMETRIC PREEJiRE was " iEl:" TORR*

1350 OUTPUT $10: ": 5$ THIE CORRECT . (IN)"

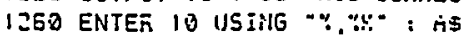

$1: 270$ OUTPUT 10

$1: 80$ IF NUM(AS) $\$$ SS THEN $1: 90$

1290 OUTPUT $0 ;{ }^{-}$CCUA

1300 ENTER D; PI I REFO EAFA

$1310 P 1=100 \cdot P 1$

1320 IF ABS(PI-51;:=5 inEN 1370

1330 OUTPUT 10 ; "THE ERTATRON IS IFT FROPERLY AOJUSTEE"

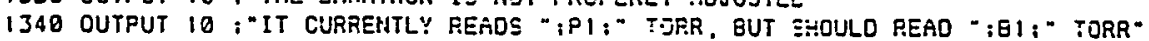

1350 GOSUB 4790 I START SLARM

1360 GOTO 5090

1370 OUTPUT D: “CC1, 320 " CLS US

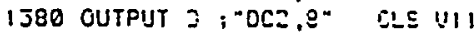

1390 WAIT $=300$

1400 OUTPUT $0:$ :DE, $4^{*}$, 2FN Uב

1410 ES=":1*

1420 GOSUB 46701 EELOW $=\{1$ SP?

$1 \neq 30$ OUTPUT $\mathrm{C}:-\left[\mathrm{CCI}, 4^{*}, \angle L \equiv \mathrm{U2}\right.$

1440 WAIT $=000$

1450 OUTPUT $D:$ :CCU4 ${ }^{-}$

1460 ENTER D: PI

$1470 P \mid=100 \cdot P 1$

1480 OUTPUT $10, "$ PRESEURE F1 - "P1:- TORR"

1490 OUTPUT $0: " 051,32^{-"}$ CPN US

:500 GOSUB $\$ 350$ I F.EAD THE EARA

IS10 P2-100*R2

1520 OUTPUT 10 : "PRESSURE PZ = " :PI:" TORR"

: 30 GOSUB 4840 I CLR $: \because @ \Xi$ SUFF

1540 OUTPUT 10 : DPEN the MANUAL :'AL'UE neares: the oot:ie on 500ce EOTTLE $n^{*}$

iS50 OUTPUT 10 : "Press :ne 'RTN' l.ey wnen tha: UALUE : : UPENED"

1550 ENTER 10 USING " : AH" : h'S

1570 IF $H 5 \$ C H R S(13)$ THEN 1530

1580 GOSUB 4390 1. FEAD THE EARA

$1590 \quad P J=100 * R 2$

1600 OUTPUT 10 ; "PRESSURE FE = ":FE;" TORR"

1610 GOSUB 4840 ! CLR TI' $\subseteq$ SUFF

1620 OUTPUT 10 ; CLOSE the MANUAL yaLUE neerss: the oo:tie on 500ic BOTTLE $A^{*}$

1630 OUTPUT 10 ; "Oress ine 'RTN' rey when the VALUE :s ELOSEO"

1640 ENTER 10 USING "...: : :\%

1650 IF KS ₹CHRS ( 13$)$ THEH 1610

1650 GOSUB 4840

1670 OUTPUT 10, "Now, OPEN the JALUE nearest :he bottle GN SO0ce SOTTLE $9^{*}$

1680 OUTPUT 10 :"Press the 'RTN" liey when thet VALUE :s BPENED"

1690 ENTER 10 USING $\%, \% K^{*} ; 1.5$

1700 IF K\$\$CHRS(I3) THEN 1660

1710 GOSUB 4390 I READ THE SARA

1750 P $4=100 . R 2$

1730 OUTPUT $10 ;$ "PRESSURE PA $=:$ P4," TORR"

1740 OUTPUT $D{ }^{-} \mathrm{OCI}, \mathrm{32}^{\circ} \mathrm{I}$ CLS US

1750 WAIT 2000 
- 2 EOSUE $4 \hat{\mathrm{a}} 40$

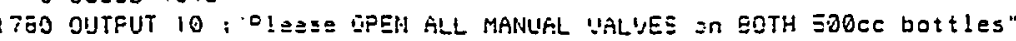

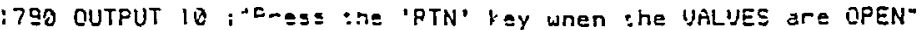

1600 ENTER 10 UEI!HG $\because: \because 4{ }^{-} ; \mathrm{r}$;

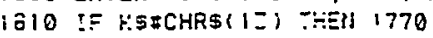

$12=0 \quad 35=-21 *$

18 OO OUTPUT $10 ;$ :DUMPIIE FO ACHEEUE TSI EETPOINT*

1940 GOSUR 4670 , EELOW TG!?

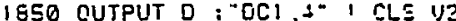

1960 SOSUB 4840

1870 OUTPUT 10 : Flase GLGSE the MANUUAL UALUES nearest the bott!e on EOTH"

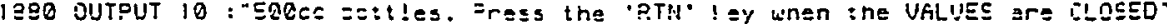

1390 ENTER 10 USIIIG $\because \ldots \%:$ :

1900 IF Y.SECHR\$(1:: - TEN : $\Xi 0$

1 Э!0 WAIT $\Xi 000$

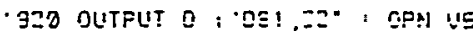

- gEO GCSUP A3SO . SËO THE ETRA

1940 DE=100+R.2

1950 GUTPUT $10 ;$ FOEEZ:IEE SE = : :PS: TORR*

1950 GOSUB 4840



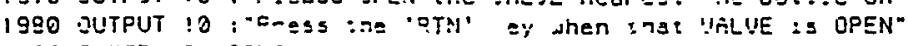

$1 \equiv 90$ EIITER 10 USIHG $\because, \because: 4:$;

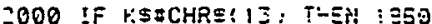

2010 GQSUB \&I90 T FE:T THE EARA

$2020 \quad P G=100 \cdot R 2$

2030 OUTPUT 10 : PEESEZFE PE = :PS: TORE"

2040 BOSUB $\div 940$

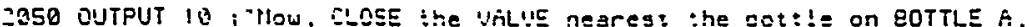

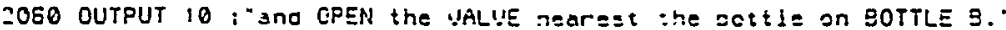

2070 CuTPUT 10 : Press :ne "aTN' 'ey snen finisneo"

2080 CUTPUT 10 e OUTfuT i 3 g gUTP!T :

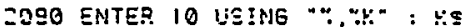

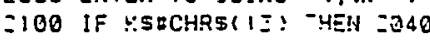

$=110$ GOEUR 4 SOD

$2 ! 20 \quad P 7 \times 100 \cdot R 2$

ZIEQ OUTPUT 10 : "PFESEUPE ET = :=7: TORR"

$=140$ SOSUE 4940

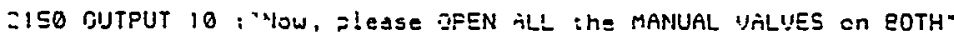

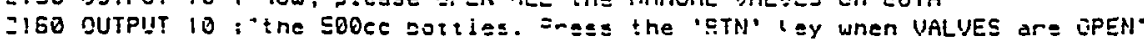

Z170 ENTER 10 USING $\because, \ldots K^{-}: 4 \$$

$2 ! 80$ IF H.SECHRS IS: THEN 2140

¿I90 QUTPUT D; 'CCI, ZZ" ' CLS US

$==00$ WAIT 1000

ZZID OUTPUT D : "DSI \&"

$2=20$ BSW-?I"

ZZZO CUTPUT :O ; -PIMPIHE TO ACHIEUE TGI EETPOIMT

$\geq 240$ SOSUB 4670

$\geq=50$ GOSUB 4840

IISO OUTPUT 10 ;"? ! ase CLOSE tha MANUAL UALUES nearest the bott le on EOTH"

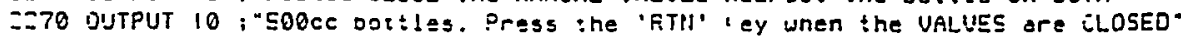

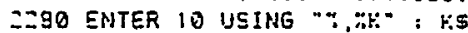

Z:OQ IF $4: 5$ CHRS( 13$)$ THEN 2250

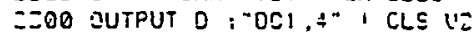

$=210$ SHIT :000

Z:20 OUTPUT 0 : $-O S 1, \Xi 2-1$ GPN US

Zइ50 WAIT 1000

ב 30 CUTPUT $0 ;$-OSZ, $^{\circ}$ I OPN UII

2350 OUTPUT D : "DCUA" 


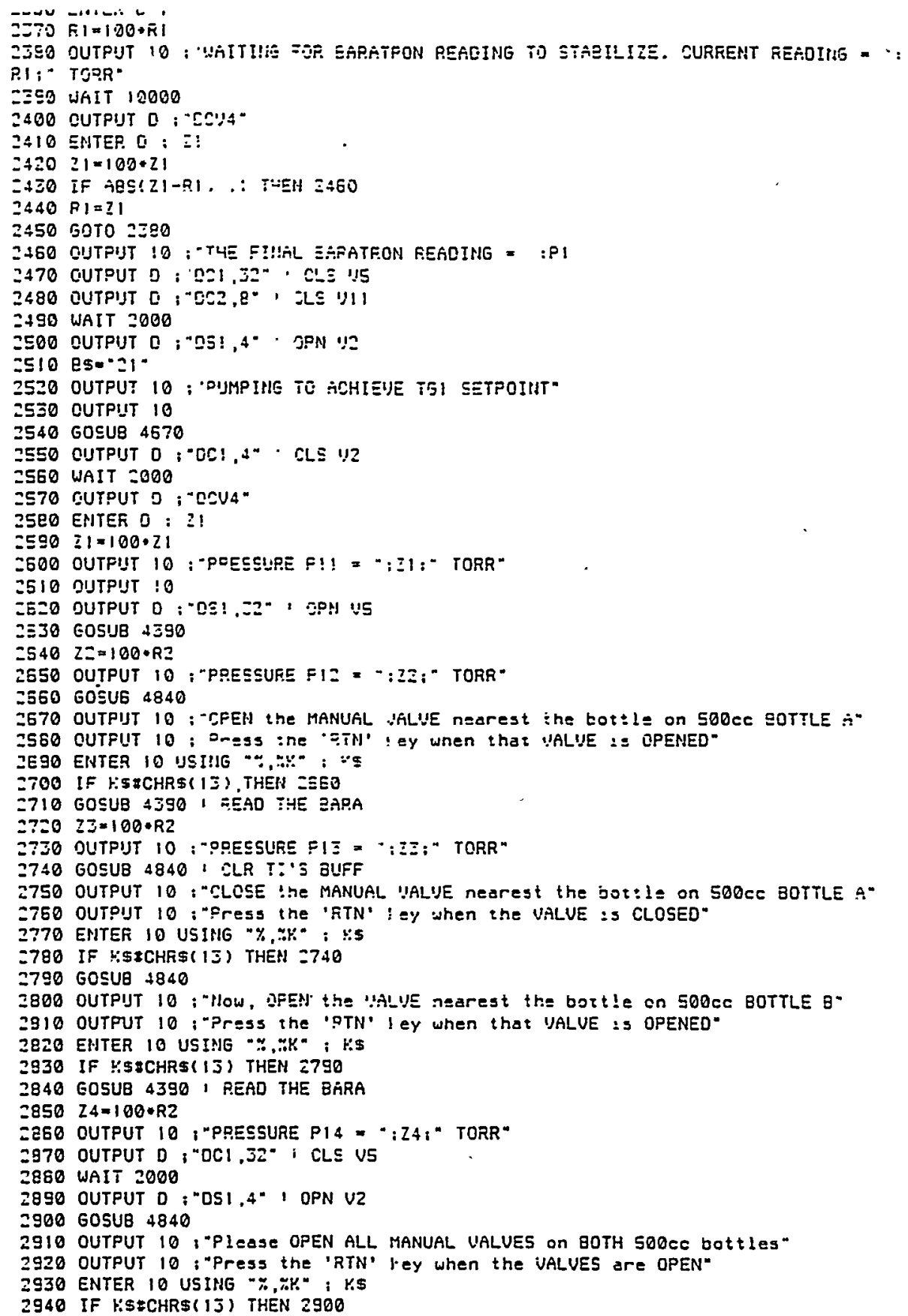


ZSË GUTFÜT 10 : 'DUMPI!!G T: SEHIEUE TEI SETPQINT"

2970 GOSUE 4670 , EE:CW T-Zi?

2980 OUTPUT $0:$ "DCI, 4 "

$2 \square 90$ GOSUB 4840

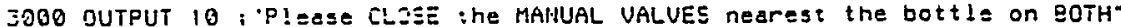

$\$ 010$ OUTPUT 10 ; 'SOOCS $25: t !$ les. Press the 'RTN' hey when the UALUES are CLOSED"

כ020 ENTER 10 USING " $\%$.

3030 iF K.S\$CHRS(13) THEN 2590

$\$ 040$ WAIT 3000

3050 NUTPUT 0 i "DSI JZ" I EN U/5

J260 GOSUE 4390 I FENT THE EARG

$2070 \quad 25=100 \cdot R 2$

ZO80 OUTPUT 10 : "FPESEUFE $\Xi: \subseteq=\cdot: Z \Xi:^{\circ}$ TORR"

三.OSO GOSUB 4640

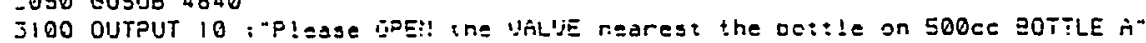



Z123 ENTER 10 USIING $\cdots, \because, K^{\circ}: \div$

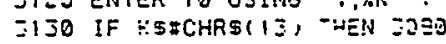

3140 GOSNE 4390 , =ESO THE EMPA

$3150 \quad 26=100 \cdot R 2$

इ160 OUTFUT 10 : "PRESELPE $=15=\cdot: 26 ; "$ TORR*

₹170 GOSUB 4840

3180 OUTPUT 10 ; "How, ZLOSE :ne VHLLE nearest the bot:le on EOTTLE $\dot{n} . "$

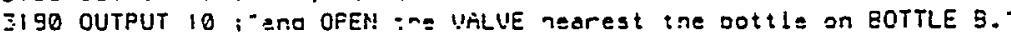

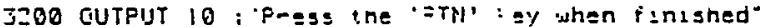

ZIO OUTFIT 10 OUTPUT $10:$ OUTPIT 10

ZZZO ENTER 10 USIHG "

JZJO IF KSECHRS(IJ) THEN ::-

3240 EOSUB 4590

IISO $\quad Z ?=100 \cdot R 2$

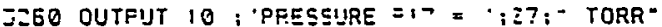



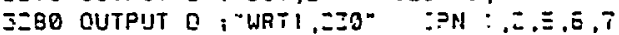

テニ90 OUTFUT 0 : “WRT:,Z,

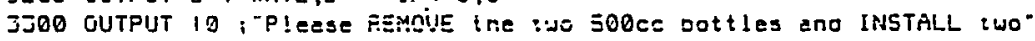

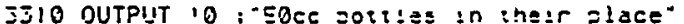

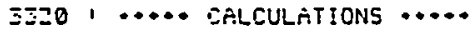

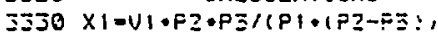

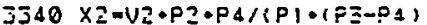

$\bar{J} 550 \times \overline{3}=U 1+P 5+P 6 ?(P A+(P 5-P 5) ;$

$3350 \times 4=U 2+P 5+P 7 /$ P P - IFE-F?

$3570 \times 5=41+72 \cdot 73 /\{71 \cdot(22-z \overline{3})$

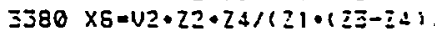

$3590 \times 7=01+25+26 i 124+135-35 i:$

$3400 \times 8=62+75 \cdot 27 /(24 \cdot 175-7711$

$\overline{2} 410 \times 9=\times 1+X 7+X \overline{3}+X 4+X 5+X 6+\times 7+\times 8$

$3420 \quad A 1=x 9 / 8$

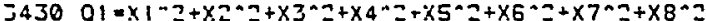

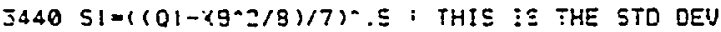

$5+50$ L $1=51+(1.96+18-1.59) /(8-2.08))$. THIS IS LE $x$

$3450 \mathrm{~L}=L 1 / 8^{\circ} .51$ THIS IS LE X EAR

$\Xi+70$ LJ=100*(LI;A1) I $*$ LE :

¿\$80 LA-100.(LI/AI), $\because$ LE $\because$ SRR

$3+50 \quad P 1=.01+1 N T(100 . P 1)$

$3500 P 2=.01 \cdot I N T(100 \cdot P 2)$

JS10 PE-.01*INT(100.PJ)

$=5=0 \quad P_{4}=.01 \cdot I N T\left(100 \cdot P_{4}\right)$

$353095=.01+I N T(100+P 5)$

3540 PE-.01-INT(i00-PE) 
Z1: …그.

ZST $22=.01+$ INT $(1.00 \cdot 2 Z:$

EEQ0 $23 \times .01+1 N T \div 100 \cdot 73$

SESD $24=.01+\operatorname{INT}(100 \cdot Z 4)$

EE00 Z5-.01-INT(100.ZE;

$=510 \quad 25 \times .01+1 N T(100.25$.

इ520 27=.01・INT(100.27:

उE3 $\times 1=.01+$ INT $(100 \cdot \times)$ :

$3640 \times 2=.01 \cdot$ INT $(100 \cdot \times 2)$

$5550 \times 3=.01+$ INT $(120 \cdot \times 5$ !

$\equiv 50 \times 4=.01 \cdot$ INT! $100 \cdot \times 4$

$=570 \times 5=.01 \cdot$ INT $(120 \cdot \times 5)$

$358076=.01 \cdot$ INT $\div 100 \cdot \times E$ ?

JESO $\times 7 \% .01 \cdot$ INT $(100 \cdot \times 7$

$3700 \times 8=.01+$ INT 1 100.X8?

3710 ล $1=.01 \cdot$ INT $(100 \cdot A 1)$

$=720=1=.01 \cdot I N T(100 \cdot 5)$

$3730 \mathrm{~L}=.01 \cdot \mathrm{INT}(120 \cdot \mathrm{L} 3)$

3740 L4=.01 +INT (100.L4)

3750 ! .... PRINT REFERT .....

5760 FOR $I=1$ TO 5

3770 OUTPIST 10

5790 NEXT I

3790 OUTPUT 10 ; THE EODE= EOTTLE EAT́ IS:*

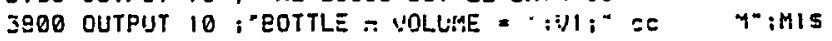

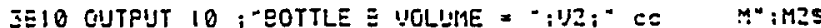

ZE20 OUTPUT 10

3E30 GUTPUT 10 ; "MEASUREO FKEEEURES:

$\$ 840$ OUTPIIT 10 :-FRESSUPE P1 10 :FI:" TORR

3950 OUTPIJT 10 : "PRESSISE FZ = ":FE:" TORR

SE60 QUTFUT 10 : PRESELFE $55=$ :PE:" TORR

FE70 CUIFIJT 10 ;"PRESEUFE F7 × iF?;" TORR"

इЕ80 OUTPIIT 10

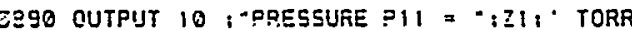

$\because 900$ OUTPUT $10 ;$-DPESSLIRE $=15=-: 730^{\circ}$ TDRR

3910 CUTPIJT 10 :-POESSURE F15 = : $25: "$ TORR

3920 OUTPUT 10 ;"PRESSURE P17 $=$ "iZT;" TORR"

3930 OUTPIJT 10

:940 QUTPIT 10 :"CALCILPTED VOLUMES: *

3950 OUTPUT $10::^{-M E A S U R E M E N T ~} 1=-: \times 1 i^{\circ} \mathrm{cc}$

3960 OUTPUT 10 : MEASUREMENT $\Xi=-: \times 33^{\circ} \mathrm{cc}$

3970 OUTPUT $10 ;$ MMEASUREMENT $\bar{\Xi}=\cdots: \mathrm{XSi}^{-} \mathrm{-c}$

3980 OUTPUT 10, MEASUREMENT $?={ }^{\circ} \mathrm{i} \times 7_{i} i^{\circ} \mathrm{ce}$

3990 OUTPUT 10

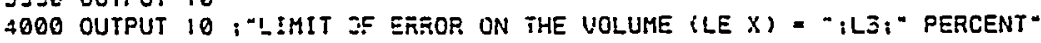

4010 OUTPUT 10

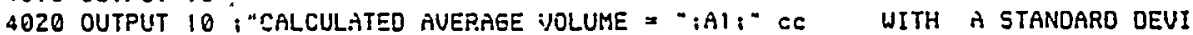
ATION OF ":SI

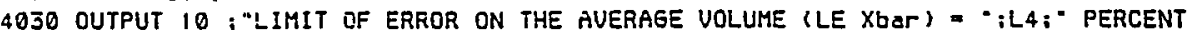

4040

4050

4050 :

4070 FOR $I=1$ TO 5

4080 OUTPUT 10

4090 NEXT I

4100 GOSUB "4840

4110 OUTPUT 10 ; "When the 50ce bott!ss have been INSTrilLED. Press the 'RTN' key" 


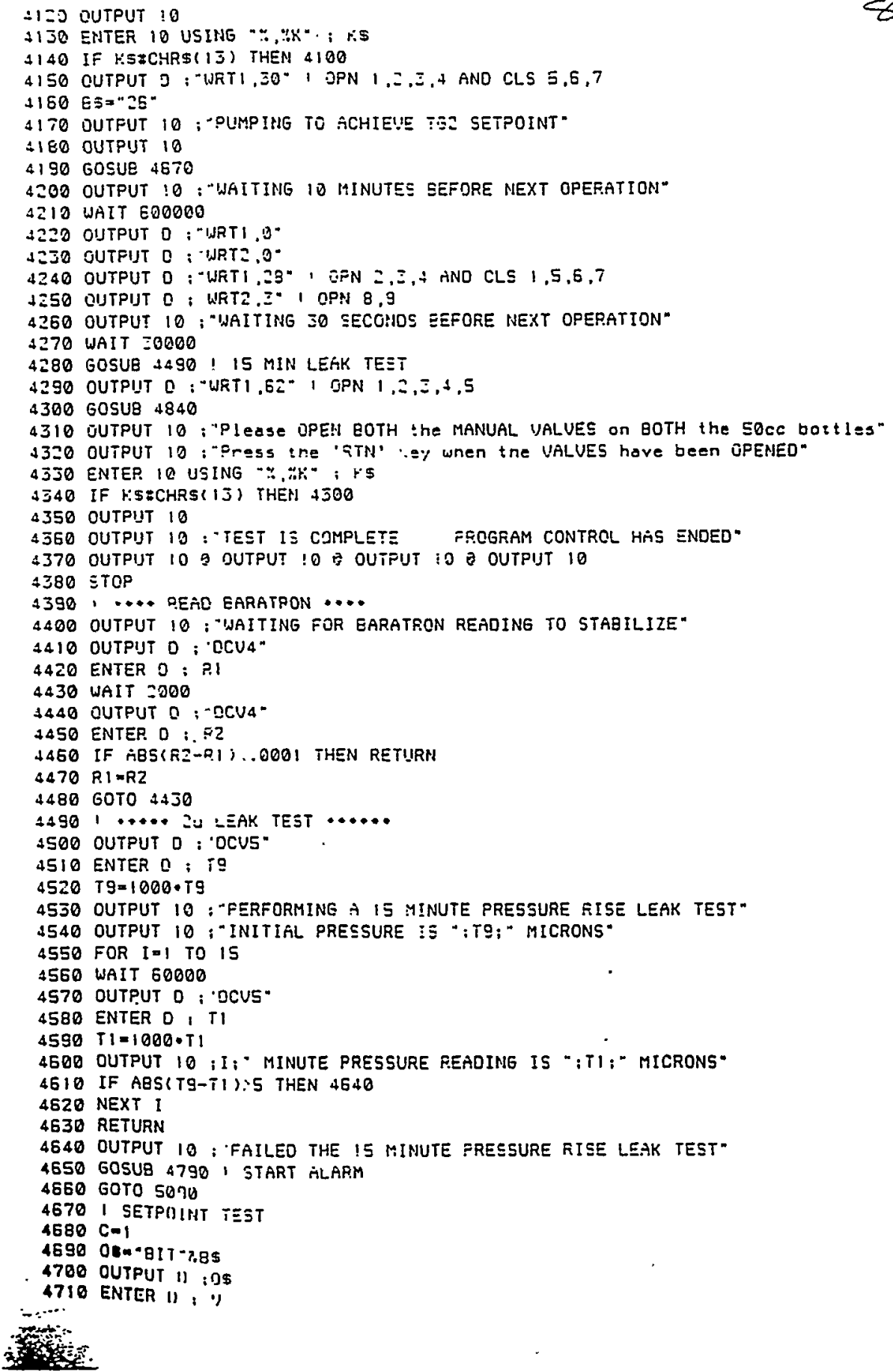


$4743:=c+1$

$4759:=0: 30$ THEN 4690

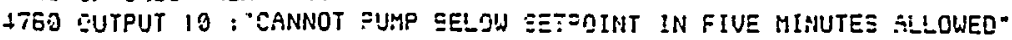

4770 STSUB 4799 I ITART ALFFM

1780 50TO 5090

4790 . ETHRT THE :LARM

4800 EUTPUT O : "CLS10"

4810 WAIT $: 90$

4820 OUTPITT $C:$ OPN1O"

4830 TETURN

4840 1 THIS CLEFFS THE T1703'

4850 EET TIMENUT $i 0 ; 1000$

4aEO SU T!!MEOU? 10 GOTO $\$ 900$

4870 ON EFROR SOSUB 5020

4880 ENTEF i' UEIHG "*.E" ;

4890 हDTO $4280^{\circ}$

4900 EET T:MEOUT $10 ; 0$

4910 GFF ERROR

4920 FEEET 10

4930 FOSUB $49 E D$ I SERIAL FORT SETUP

4940 FETUFM

4950 - EERIPL FORT SETUP

4960 COHTROL $10.4 ; 25$

4970 CONTROL $19.9 ; 139$

$\$ 980$ CONTROL $10.11 ; 194$

$\$ 990$ CONTRCL $: 0,12: 13$

5000 CONTROL :0,14:17

5010 SETURN

5020 I SERIAL SORT ERROR $\Xi / R$

5030 SEF TIMEQUT 10:0

5040 SFF ERROR

5050, EPROR = !:4 !5 EUFFER RUEF.TUN

S060 IF ERPN $=114$ THEN RETURN

5070 CUTPUT 10 : "EERIAL FORT EFROR IUUMEER ":ERRN" OCCURRED AT LIME ";:TFL

$5080 \leqq T O P$

$5090, \ldots \ldots+$ SHUTOOWN $\ldots . . .+$.

5100 CUTFIT $\Omega$ : -WRTI , $0^{\circ}$

5110 CUTPUT Q : 'WRT: $0^{\circ}$

5120 SUTFUT D : "WRTI. $.22^{*}$

5130 CUTPUT O ; "WRTE.3

SI40 CUTPUT 10 ; PROGRAM CONTROL IS EEING TERMINATED-

5150 OUTPUT 18 OUTPUT 10 Q CUTFIJT 100 OUTPUT 10

5160 STOP 


\section{APPENDIX E: FLOWCHART FOR MODIFIED THERMAL}

DECOMPOSITION SOFTWARE 

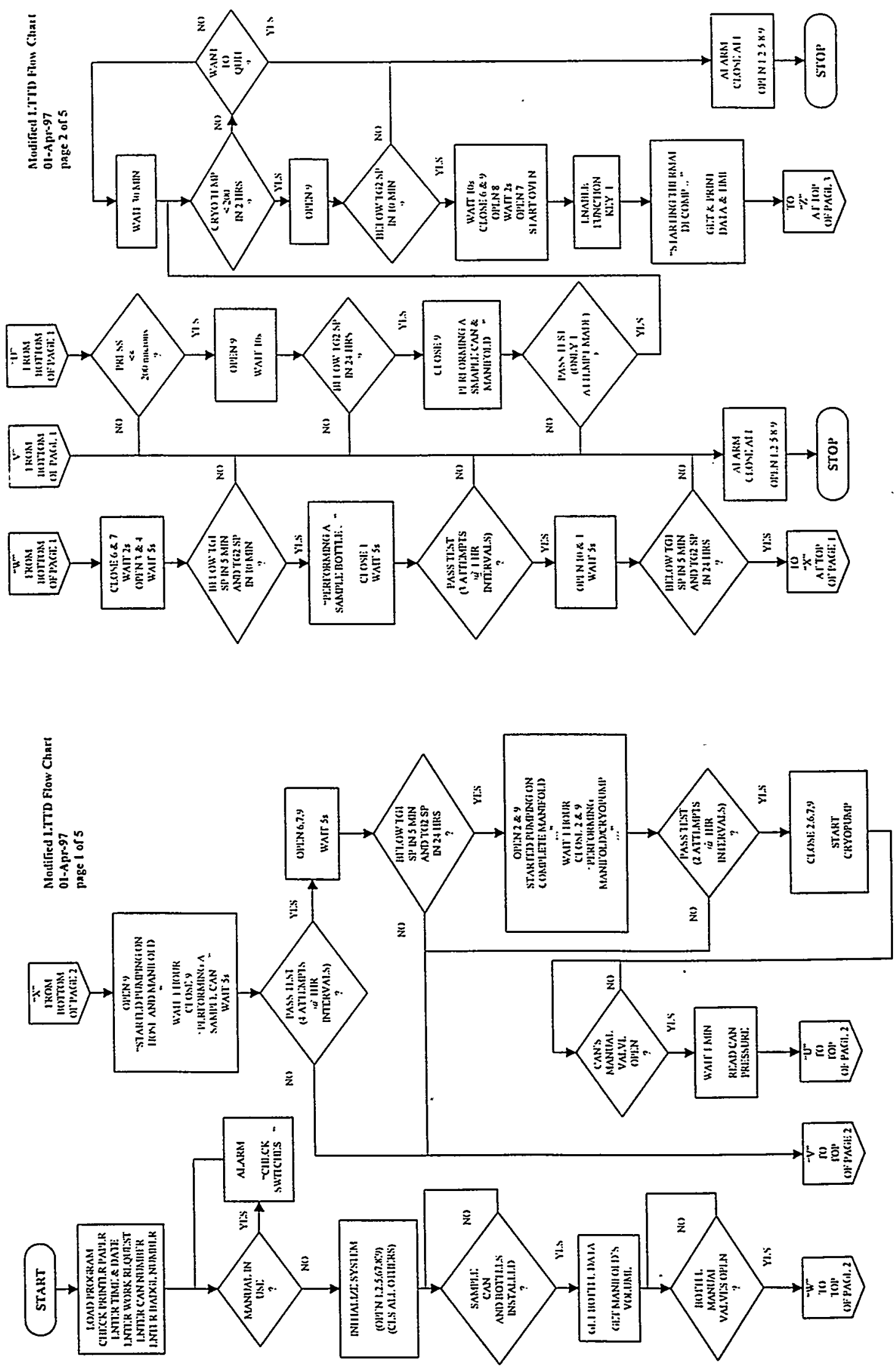

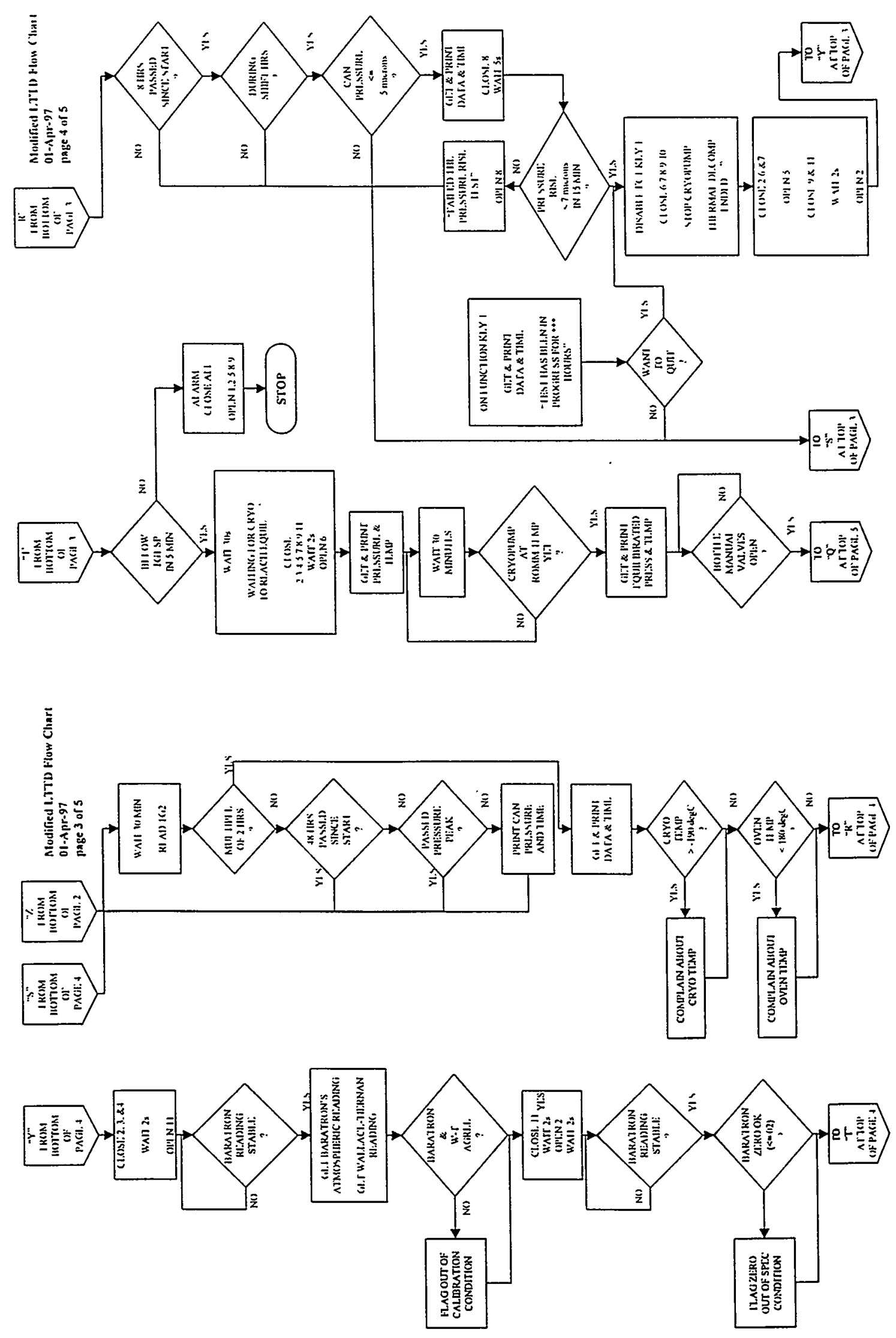


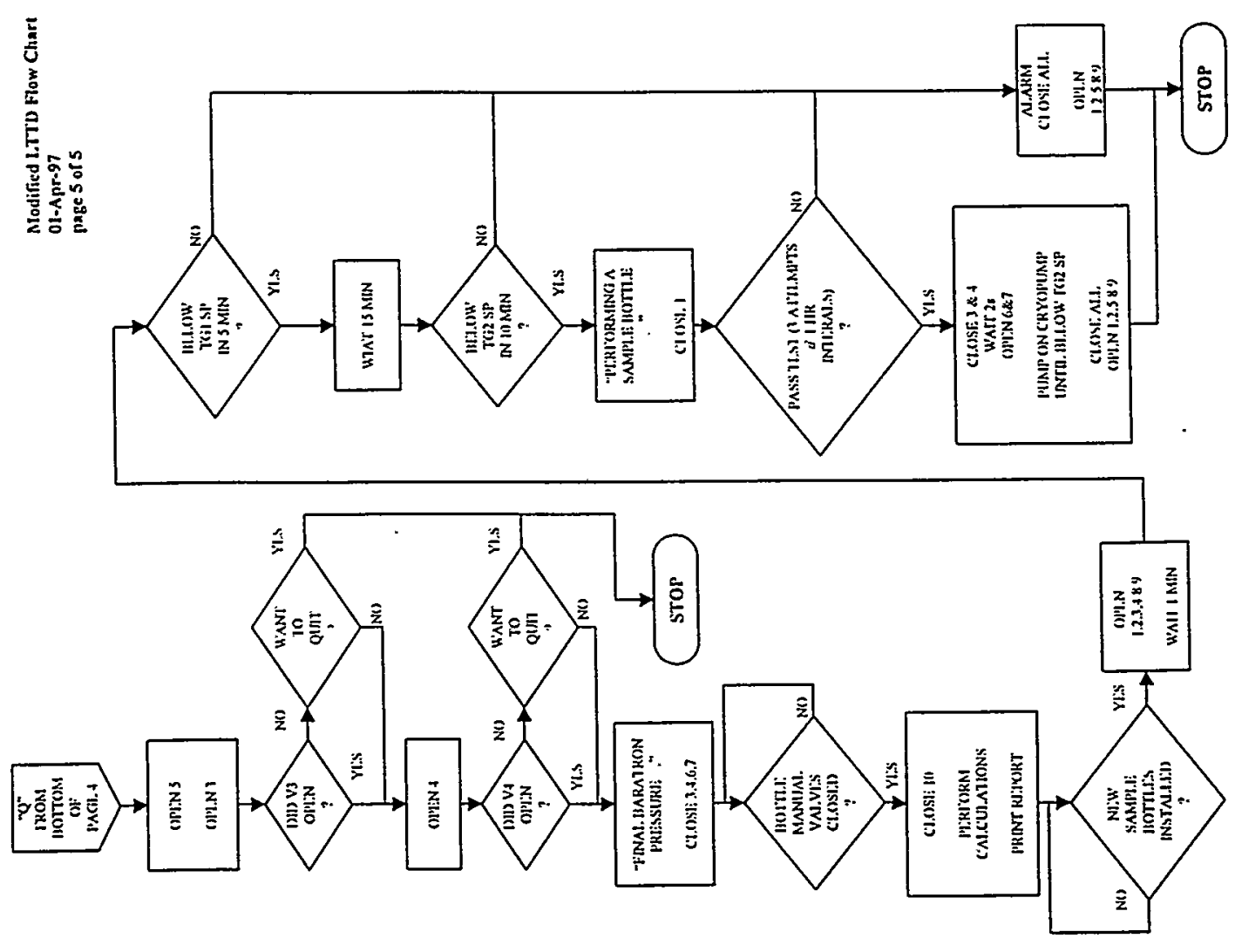




\section{APPENDIX F: HARDWARE DESIGN FOR ENHANCED LTTD WORKSTATION}

Note: The material in this appendix corresponds to the detail sections of the Engineering drawing package titled Enhanced LTTD Workstation. The Y-12 Engineering drawing numbers associated with this package are E2E92042EA133 through E2E92042EA140 inclusive.

Any discrepancy between the drawing package and the material in this appendix shall be resolved in favor of the drawings. 
Enhanced Low Temperature Thermal Decomposition (LTTD) Workstation Documentation Key, Rev O, REI 980210

\begin{tabular}{|c|c|}
\hline System Cabling & Major Modules \\
\hline $\begin{array}{l}\text { Connectionolagran, } \\
\text { cableIdentseicatien }\end{array}$ & 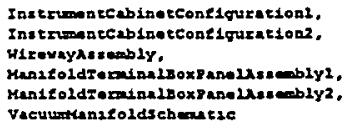 \\
\hline
\end{tabular}

Other System Documentation

Poverrequszanents,

ILITDVArtiLiat,

Instxcabls acmbly ras truetions.

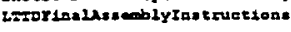

Instrument Cabistet Detail

Vacuum Manifold Detail

OLg2ta10utputzerminalelock10erazl. Diqies1Outputtermenalalock2Datasl. Araloglaputzeminals LockDotail.

OLg2talinputzemanelslockoete11.

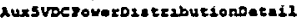

Rux24VDC7oveross tzibutzonDetel1.

ss2327ntarzacedotasl,

zurbopuroproqCodyertazDotall.

Cryopumioraporaturabsoplayoetald.

Earatzonoloplaydoek11.

Convectronolaplayoser.

Sonaurgab1splayootasl

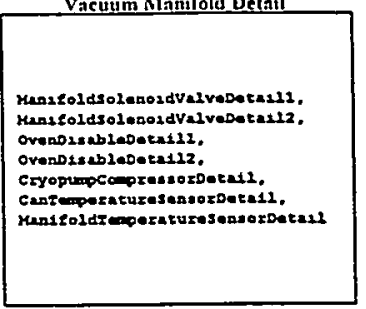


Enhanced Low Temperature Thermal Decomposition (LTTD) Workstation Connection Diagram, Rev A, REI 980115

Instrument Rack Vacuum Manifold \& Grieve Oven

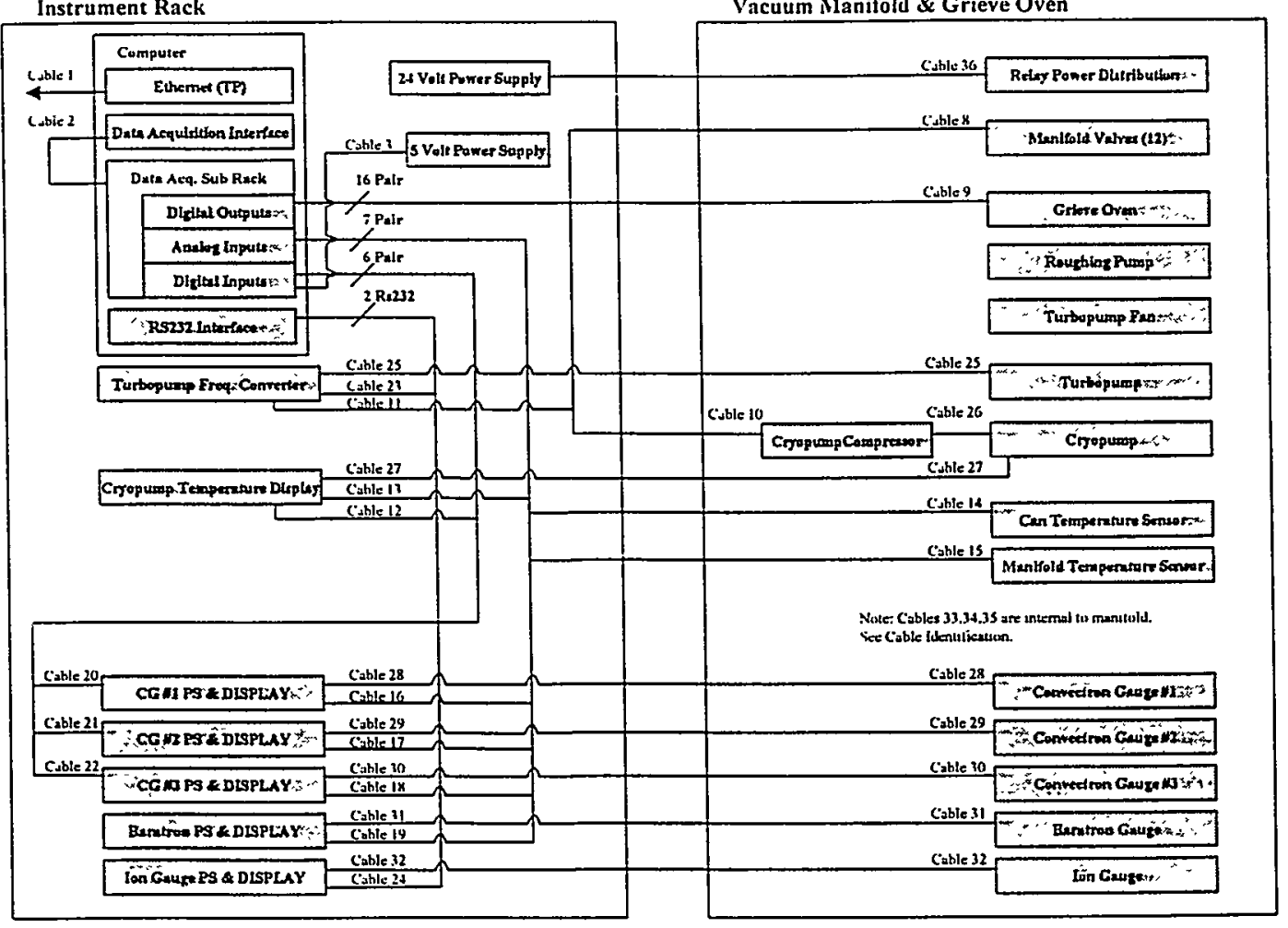

Enhanced Low Temperature Thermal Decomposition (LTTD) Workstation Cable Identification, Rev A, REI 980210

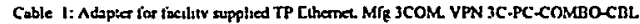
ree vendur supplied dxearnenteruon.

Cuble 2: Control cable between Dats Acquistion Intertiace and Dain Acyurstion Sub Rech

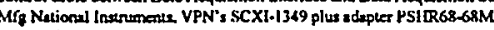
res iendur supplied ducementation.

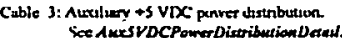

Digies ouput ignals.

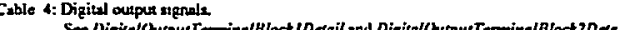

See Dixidellowput Tom

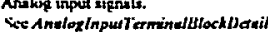

Cable 6 Digitul enpur signals.

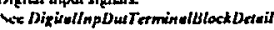

C'able 7 RS232 channels. the RS2JIIniafucr Deail.

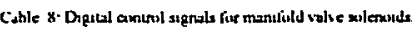

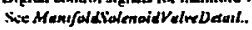

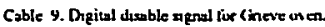

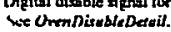

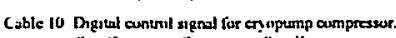

Sce Cimopumplempressoridenth

Cable II: Drgatal cuntrol ngenl ior turbopump firequency conventer. See Turbapuspof Freg Converer Detsil.

Crble 12: Digitul urtper signals (setpoints) For Cryopump Tempetature Montor.

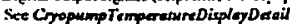

Cable 13: Ana log whiput s1gnal for crįupurnp lemperature display.

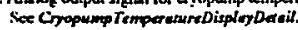

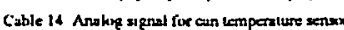

See ConTerperumsesonserbaeil

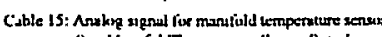

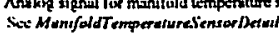

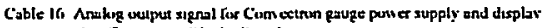

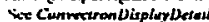

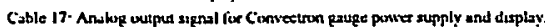
ter Conmerrandirplop Dereil.

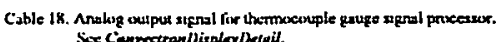

Cable 19: Anslog outpus signal for Beratron signal processor.

tee Berarron Dignion Deasid.

Cable 20: Digitel output signal (setpount) frum Cunvectron geuge power supply and dusplay. See Carmetrom DirplopDesis.

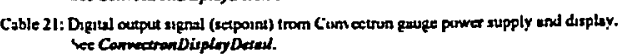

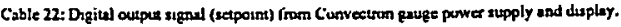
Sice Cosmoctron Displey Dectil.

Cable 23: RS232 channd lur turbo purap Irequiney cumsener.

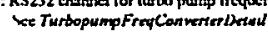

Cible 24: RS2.12 chraned tor kn qauge.

$x$ lanGanke Dicplay Deaul.

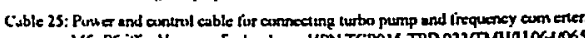

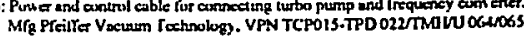



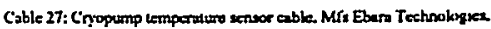

Cable tix VPN 917 A0085

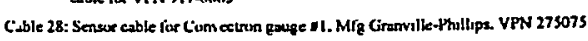

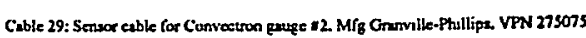

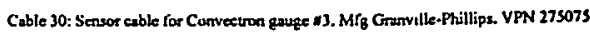

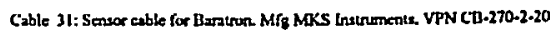

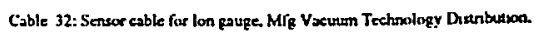
VPN L64.032S-DCCIII

Cable 33. Contrul able tar Onan Ineable Relav. use OnenDisebic Derail

Cable 3t Cuniral cable liar Conupump trable Relay.

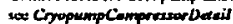

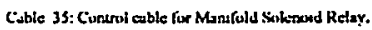

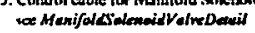

Cable 36: +24VDC panver and pentror senescoble

se ManifoldTerminel BaxPanelhusembly 
Enhanced Low Temperature Thermal Decomposition (LTTD) Workstation Instrument Cabinet Configuration, Sheet I of 2, Rev A, REI 970815

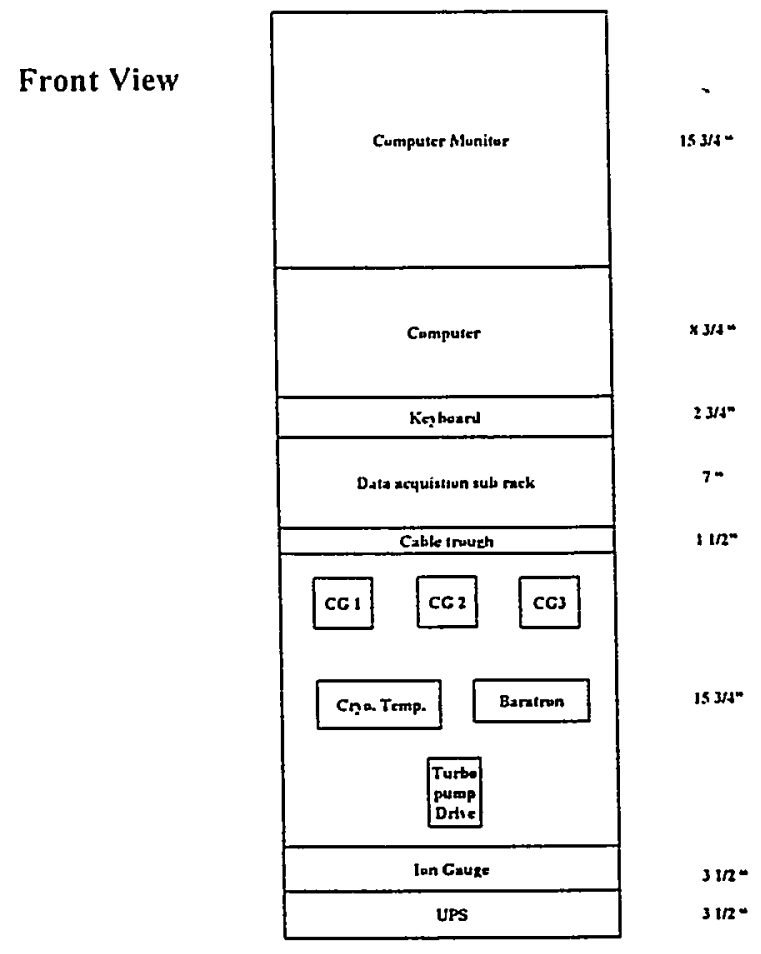

Enhanced Low Temperature Thermal Decomposition (LTTD) Workstation Instrument Cabinet Configuration, Sheet 2 of 2, Rev A, REI 980815

Rear View

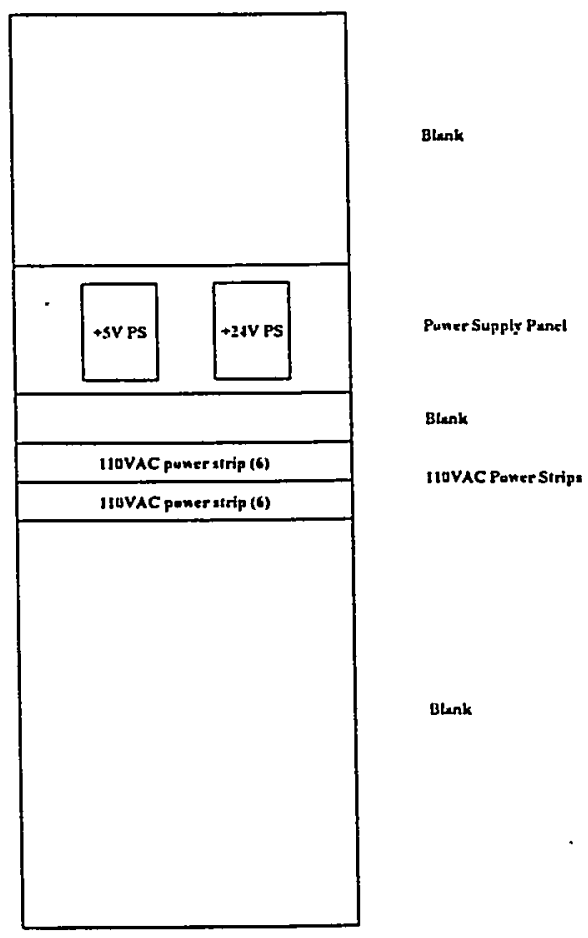


Enhanced Low Temperature Thermal Decomposition (LTTD) Workstation Wireway Assembly, Rev O, REI 970711

\begin{tabular}{|c|c|c|c|c|}
\hline 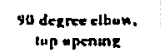 & Rat exalinz plate & $366^{6}$, tratikht & rat seating patse &  \\
\hline  & & & & fat cesting platic \\
\hline 12 "unient & & & & 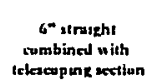 \\
\hline 90 degrese exaling plater & & & & 90 degrere seating platec \\
\hline Bux Connector & & & & Box Connecter \\
\hline
\end{tabular}

Parts List for 2.5x2.5 "Lav in" Wireway

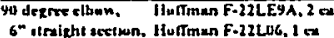

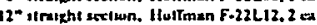

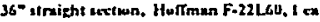

Iclecope eset. (1-7-1), Hu liman F-22LA, $2 \mathrm{CO}$

AI sesthe plate, Hutmenn F-22LSCQR, 2 ex pkg(s)

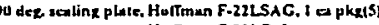

HurTman F-22LB.3

Wireway Cross-section Detail

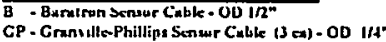
C. - Perkin Elmer lun Gauze Sensur Calle - OD 1/1/"

TP - Pfiefter TurbuPump Cunirul Cahle - OD 1/2" TC - Type "J" Thermecuuple W/re - OD $1 / \mathrm{s}^{n}$ RTD- RTD Cuble - OD in

CT - Cnupump Temperature Sensur Cable - OD 1/4

DC. Digital Cuntrul Cable. ov $3 /$ th

2AV - 2AVDC Puner Cable OD 114"
SP . Spare shiclused pair miret . OD

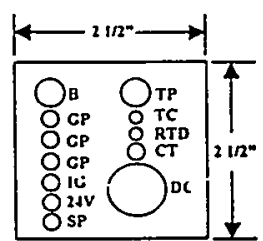


Enhanced Low Temperature Thermal Decomposition (LTTD) Workstation Manifold Terminal Box Panel Assembly, Sheet 1 of 2, Rev A, REI 980204

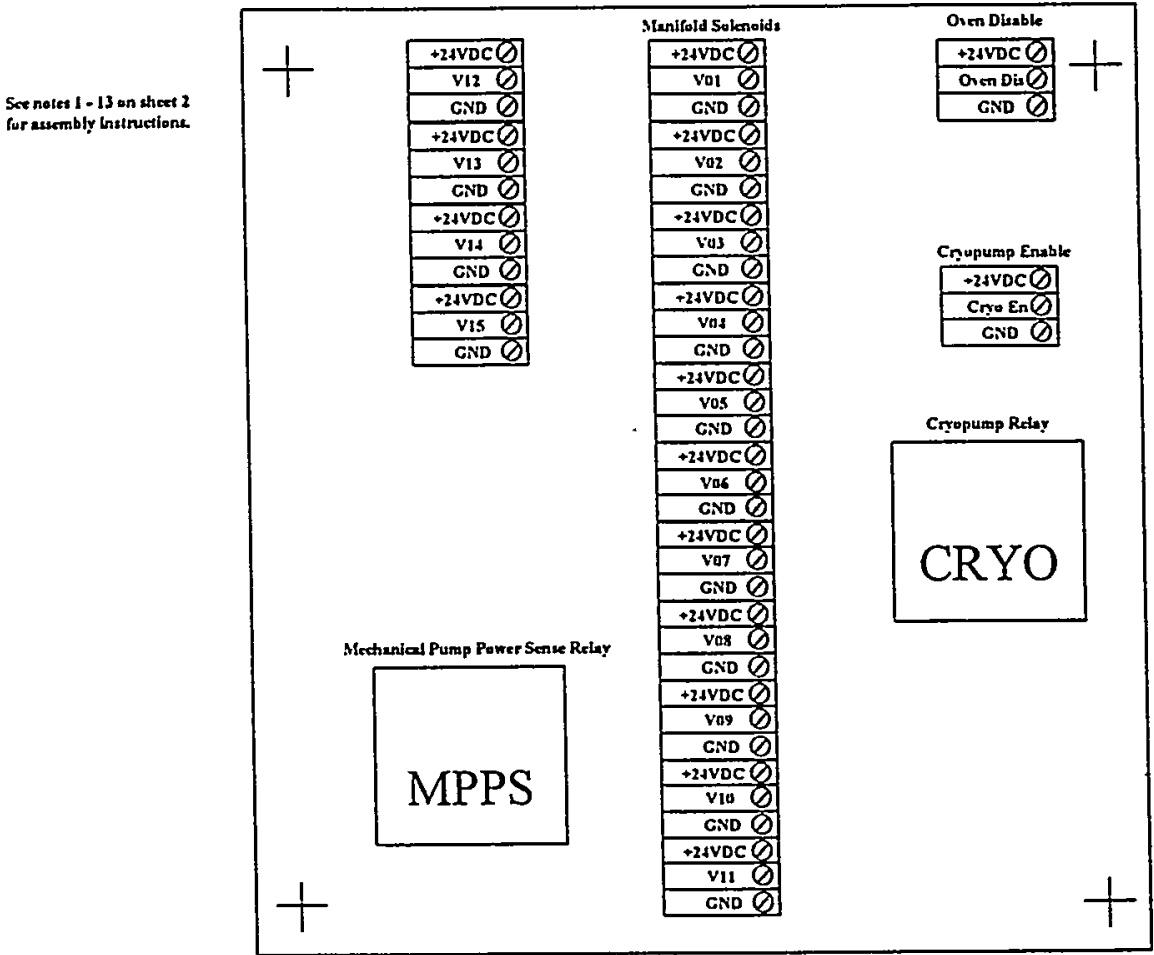

Enhanced Low Temperature Thermal Decomposition (LTTD) Workstation Manifold Terminal Box Panel Assembly, Sheet 2 of 2, Rev A, REI 980115

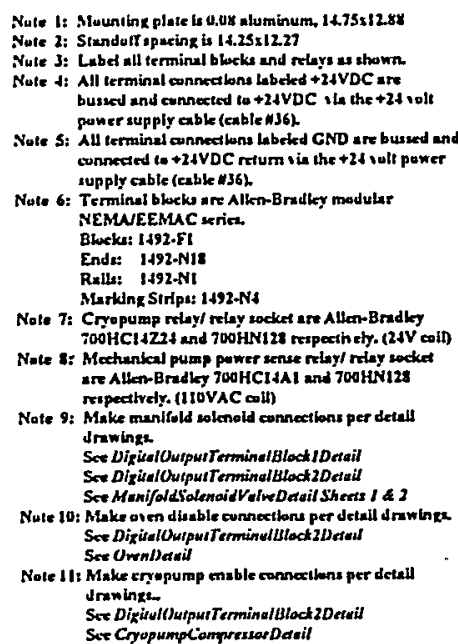

Nate 12: The folluwing abla are routed throuth the menifuld

terminal bos

Turbapurap power and control cable (1625)

Cryopump lempernature weneor cable (1127)

Cxn teaperature senior cable (WIt)

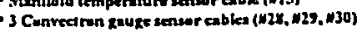

- Baratron kau ze senver cable (1JI)

- lon quU ze secruor calle (MJ3)

Nute 13: 110 VAC pawer cable foum the sures feeding

the mecturieal pumap entere the meniluld terroinal bes and drive the input of the mectrasical pump porrer sansorsty. 
Enhanced Low Temperature Thermal Decomposition (LTTD) Workstation LTTD Vacuum Manifold Schematic, Rev O, JAB/REC 970922

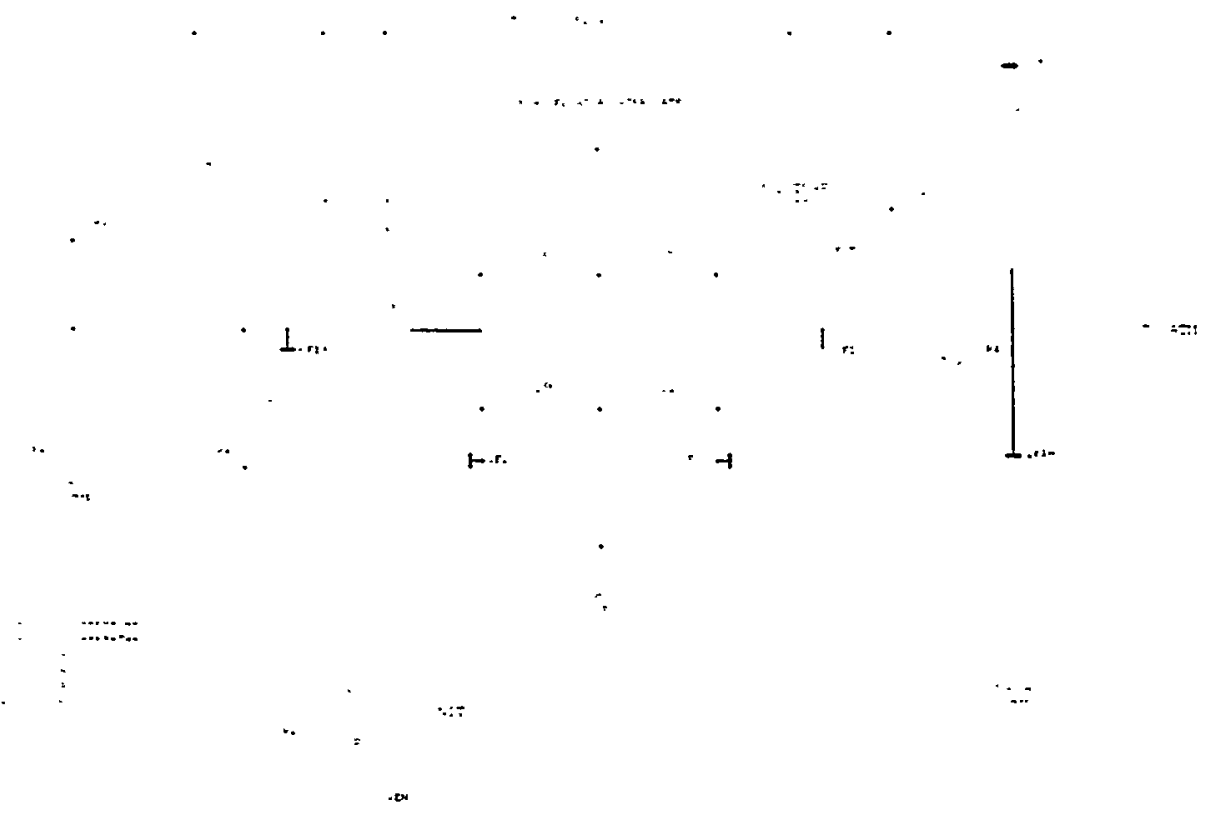


Enhanced Low Temperature Thermal Decomposition (LTTD) Workstation

Power Requirements, Rev A, REI 980110

Instrument Rack

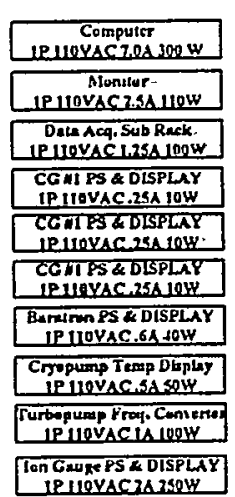

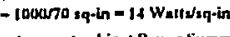

i2 uutkets IP IIUVAC, I 30 Amp circuit
S Voll Powor Supply

-10\%

24 Yolt Parer Supph

ilow tgp -
Vacuum Manifold \& Grieve Oven




Enhanced Low Temperature Thermal Decomposition (LTTD) Workstation

Instrument Cabinet Assembly Instructions, Rev O, REI 970628

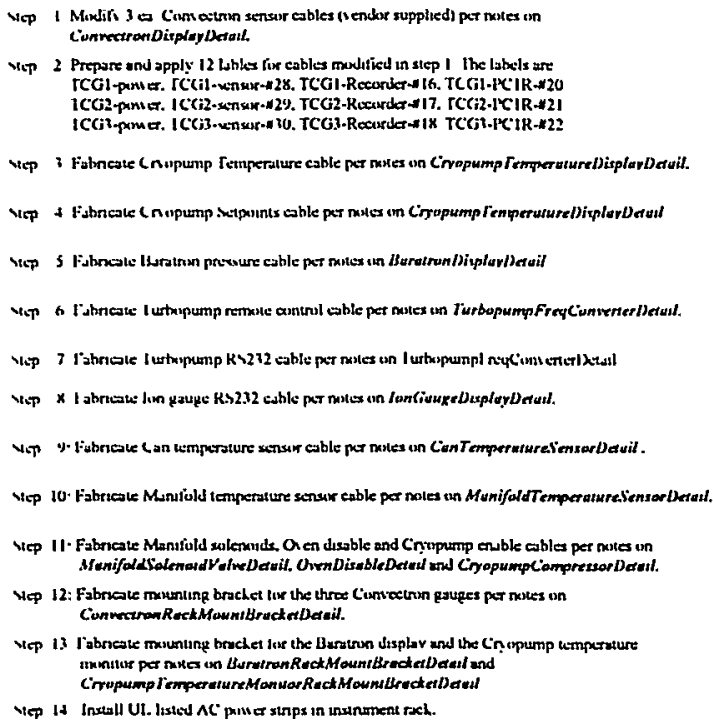

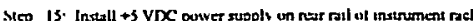

Vep 16. lohneate +5 VDC pawer able per nxtes un DCPower DistributionDerent.

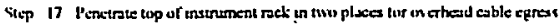


InterumentRack AstembirDiaxrem.

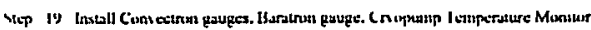

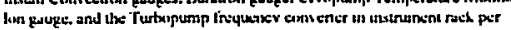

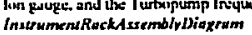

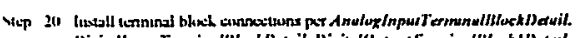

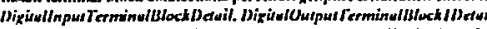
Ming

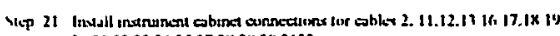

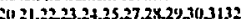

\section{Enhanced Low Temperature Thermal Decomposition (LTTD) Workstation}

\section{LTTD Final Assembly Instructions, Rev O, REI 970711}

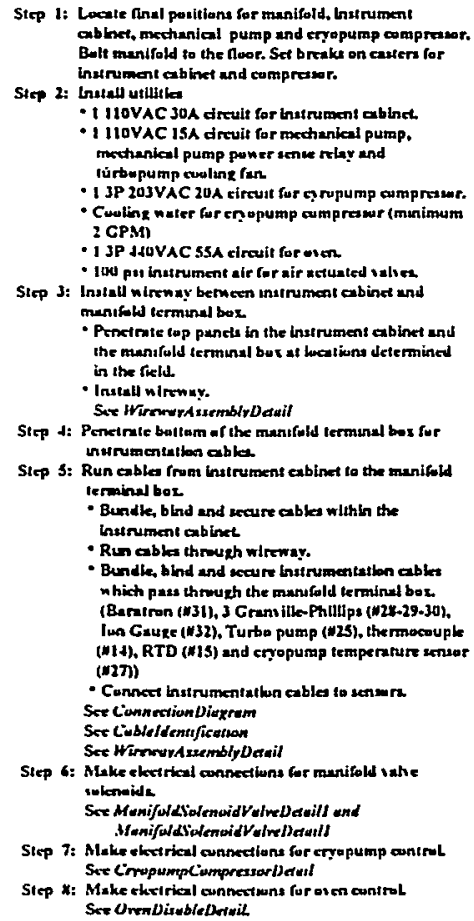


Enhanced Low Temperature Thermal Decomposition (LTTD) Workstation

Digital Output Terminal Block 1 Detail, Rev O, REI 970711

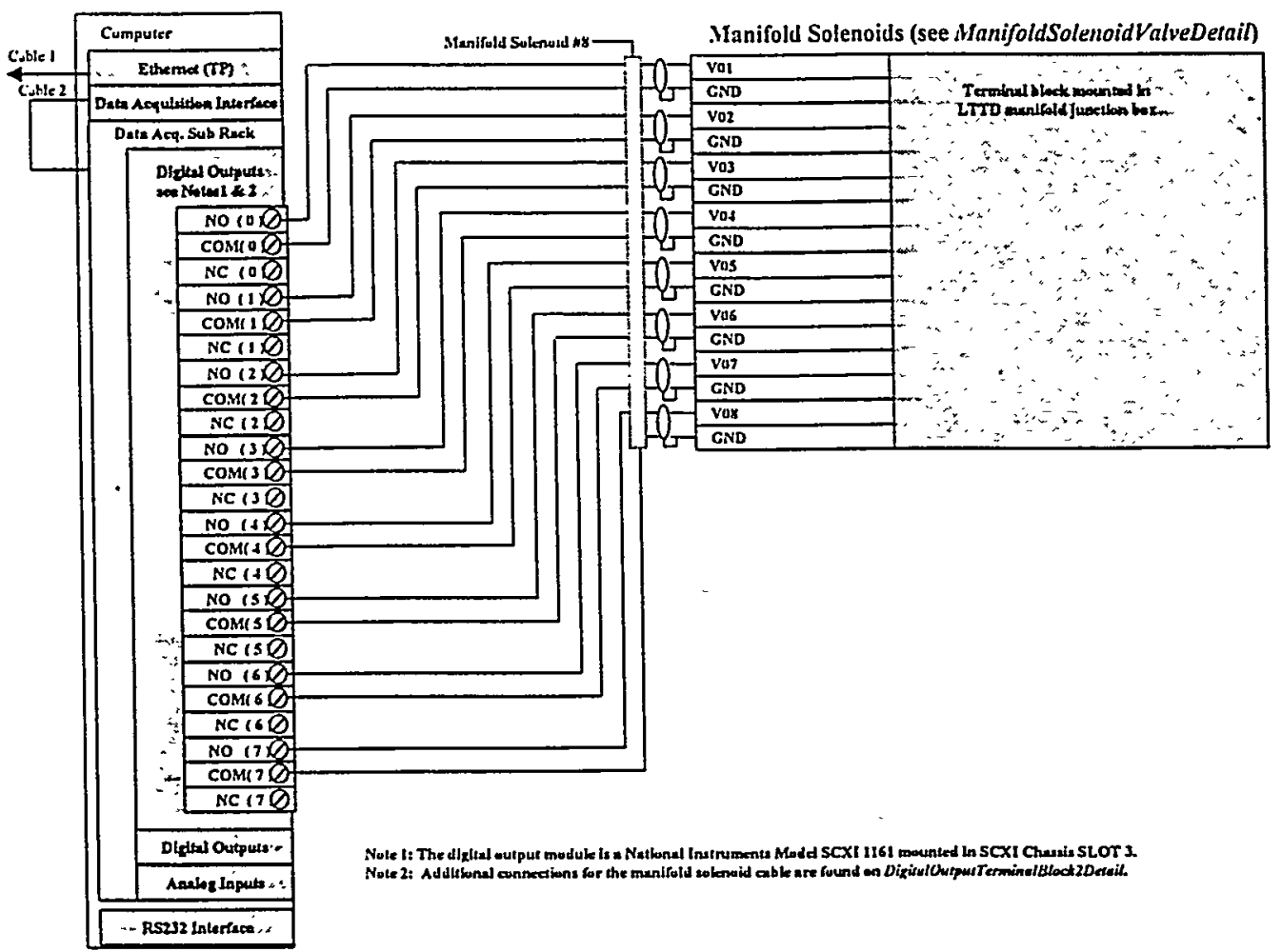

Enhanced Low Temperature Thermal Decomposition (LTTD) Workstation Digital Output Terminal Block 2 Detail , Rev O, REI 970711

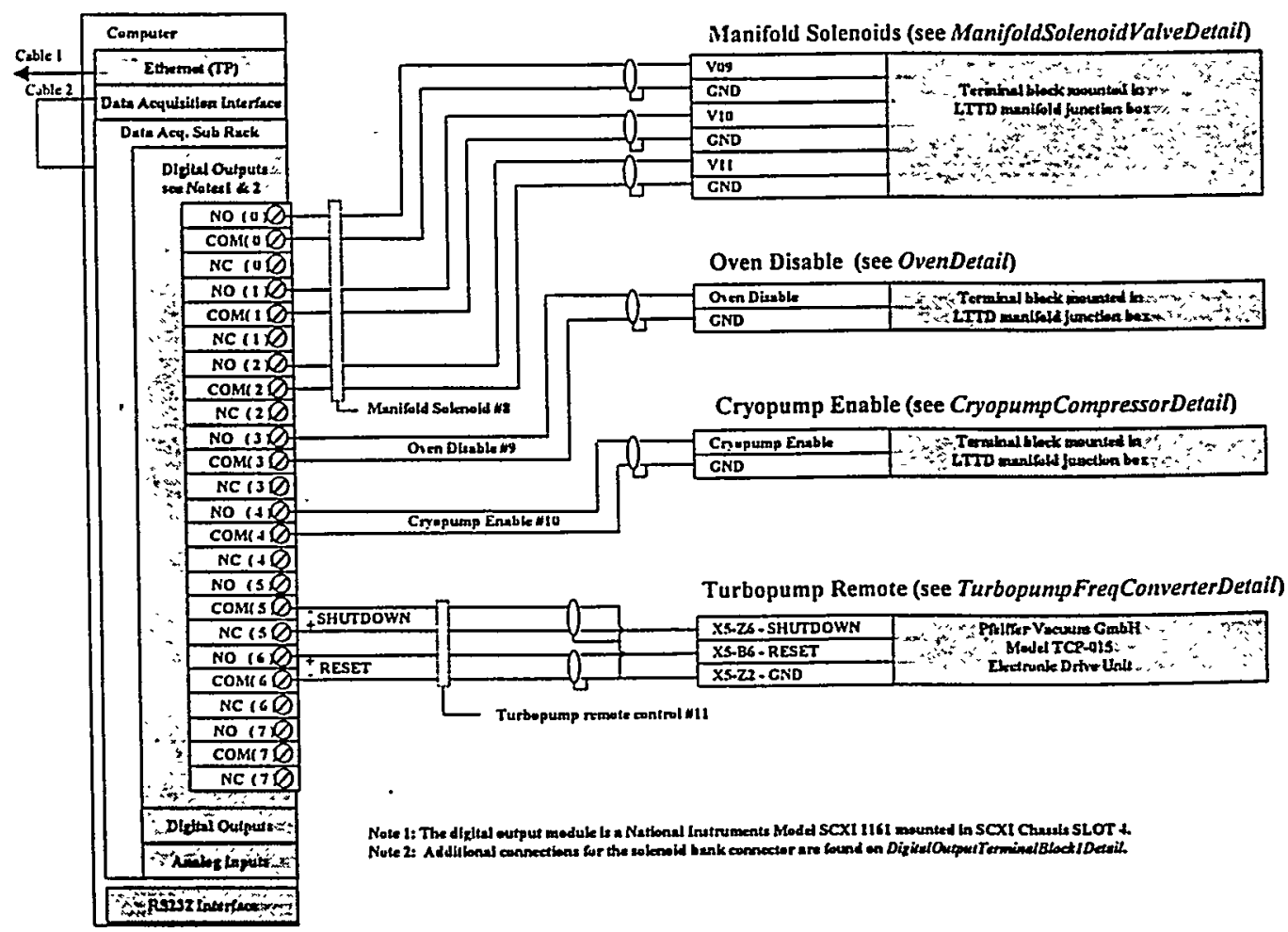




\section{Enhanced Low Temperature Thermal Decomposition (LTTD) Workstation}

Analog Input Terminal Block Detail, Rev O, REI 970711

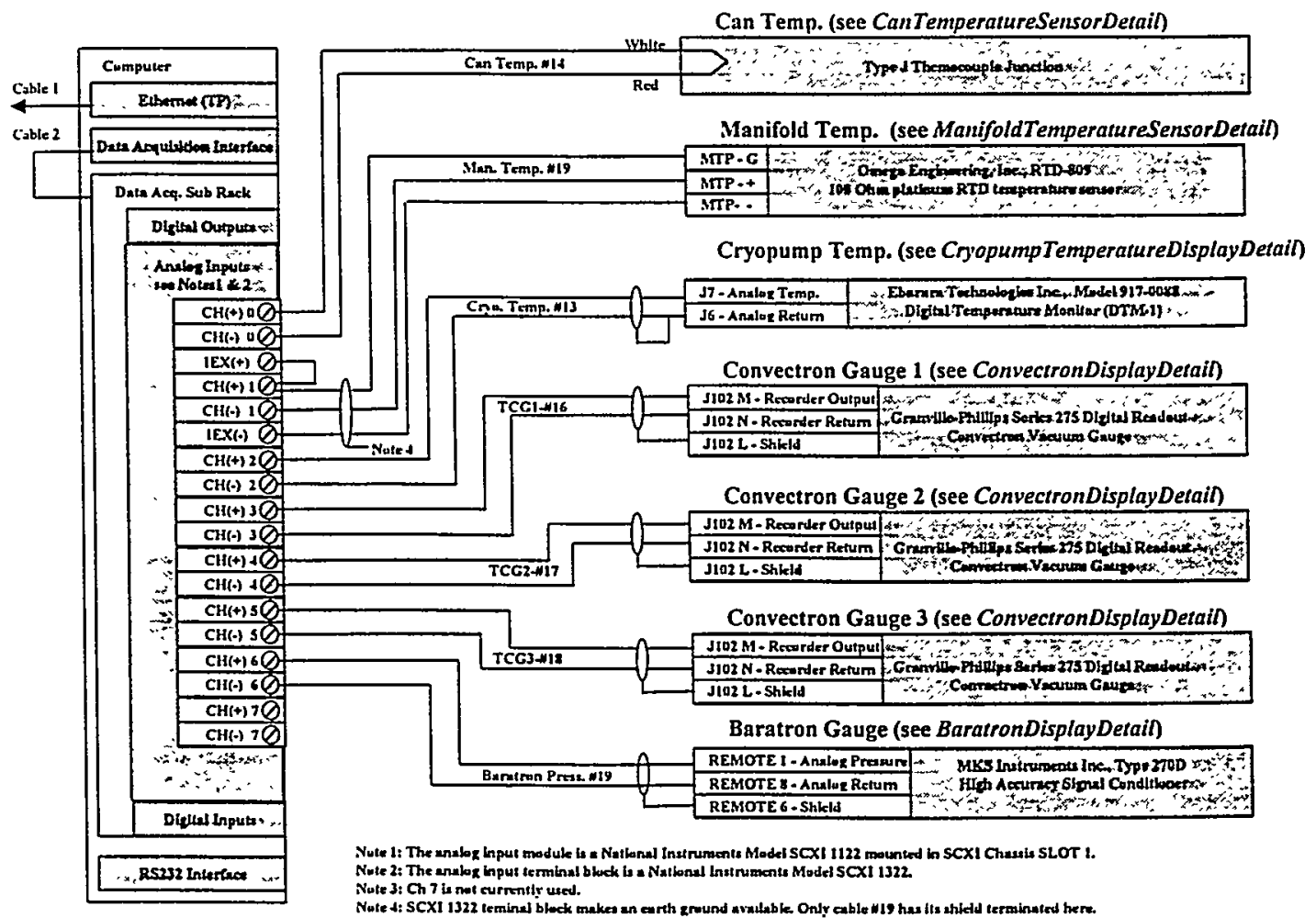

Enhanced Low Temperature Thermal Decomposition (LTTD) Workstation Digital Input Terminal Block Detail, Rev O, REI 970711

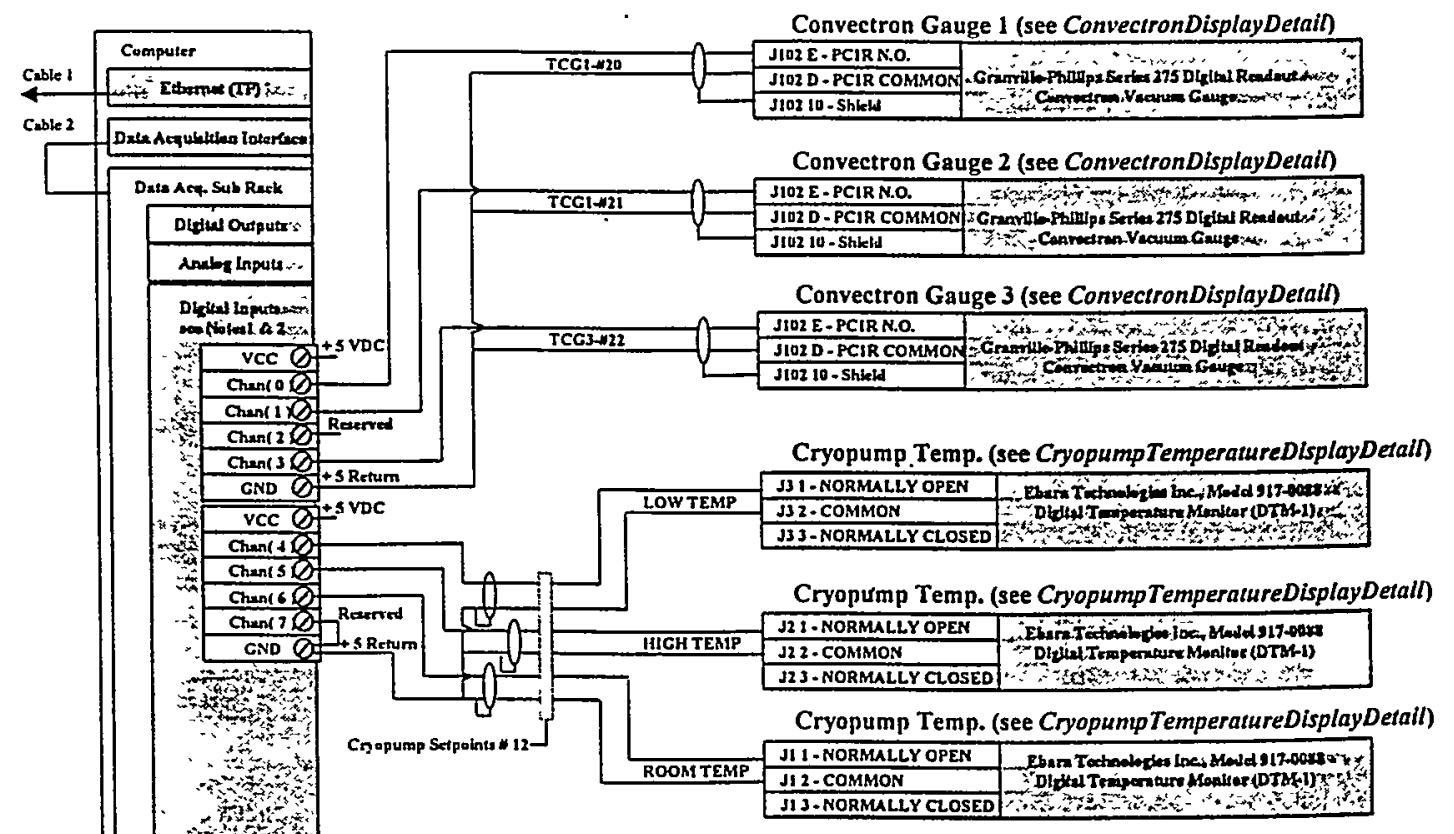

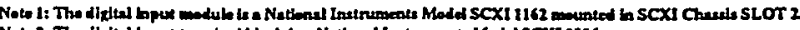

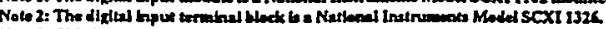

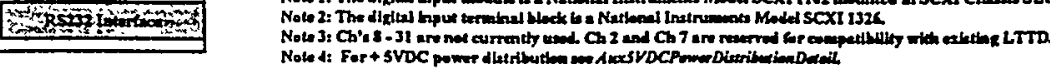


Enhanced Low Temperature Thermal Decomposition (LTTD) Workstation Auxiliary +5 VDC Power Distribution Detail, Rev O, REI 970711

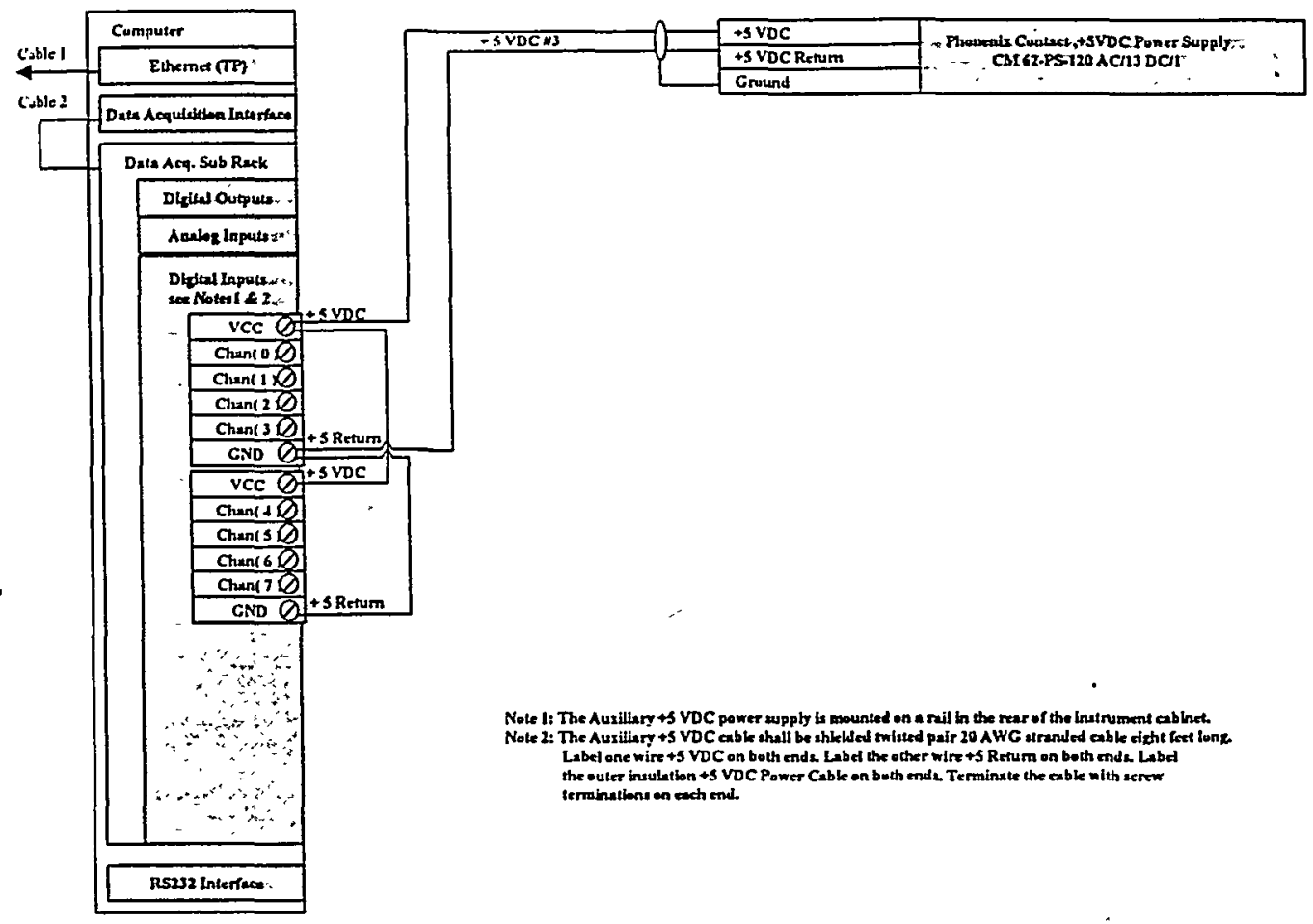

Enhanced Low Temperature Thermal Decomposition (LTTD) Workstation Auxiliary +24 VDC Power Distribution Detail, Rev O, REI 980115

val

\begin{tabular}{|c|c|c|c|}
\hline $24 \mathrm{vDC} 0$ & & -21 VDC & LA, +2uYDCPunt \\
\hline Voi O & + 2f VDC Pawer Cable IJ6 & Ground & $\therefore$ CA125-PS-120-2301CC21DCS: $\therefore$ \\
\hline
\end{tabular}

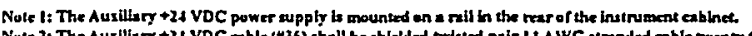

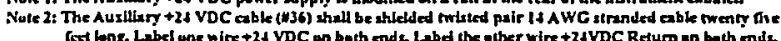

Fest bong, Lebel une wire +24 VDC un bath ende Lebed the other wire +24VDC Return en beth endx.

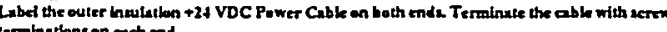

termatrations on each end. 
Enhanced Low Temperature Thermal Decomposition (LTTD) Workstation RS232 Interface Detail, Rev O, REI 970711 .

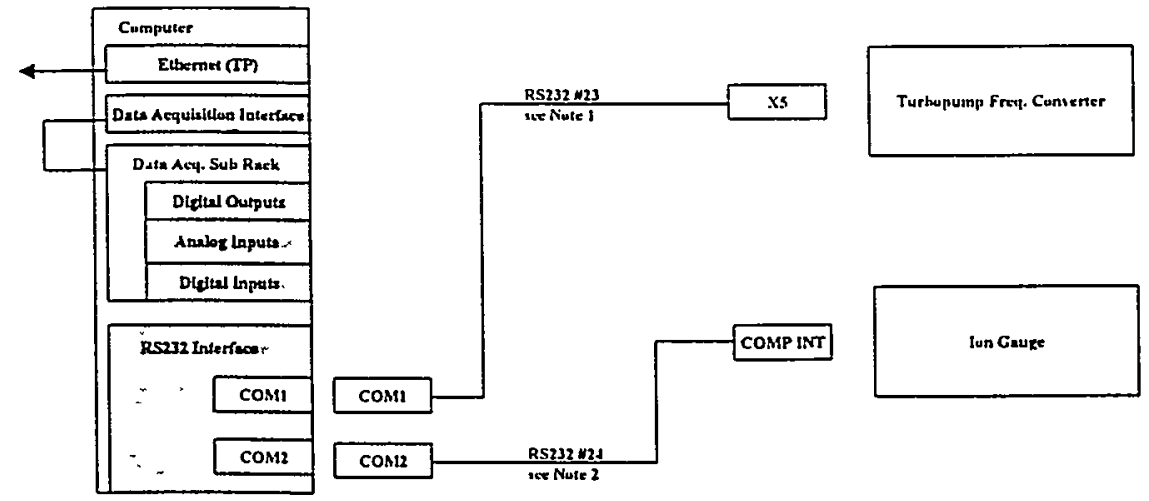

Note 1: Turbopump RS232 cable shall be shielded 3 conductor 22 AWG stranded eable eight feet long. Lalel the calle "Turbopump RS232 323 . Altach one end of the cable to a nine pin female type " $0^{n}$ subminature connector. Lable it COM1. Attach the other end to the vendor supplied connector per the vendor's requirements.

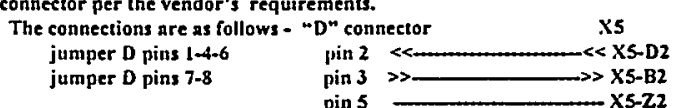

Note 2: Ion gauge RS232 cable shall be shielded 3 conductor 22 AWG stranded cable cight feet long. Label the cable "Ion gauge RS232 H24. Attach one end of the cable to a nine pin ferale type "D" subminature connector. Lable it COM2. Attach the other end to a 25 pin male type "D" subminature connector. Lable it COMP. INT.

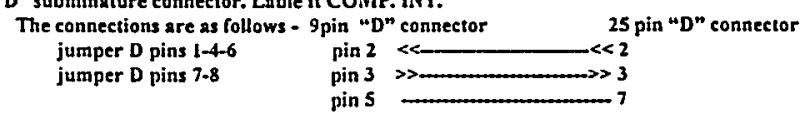

Enhanced Low Temperature Thermal Decomposition (LTTD) Workstation Turbopump Frequency Converter Detail, Rev O, REI 970711

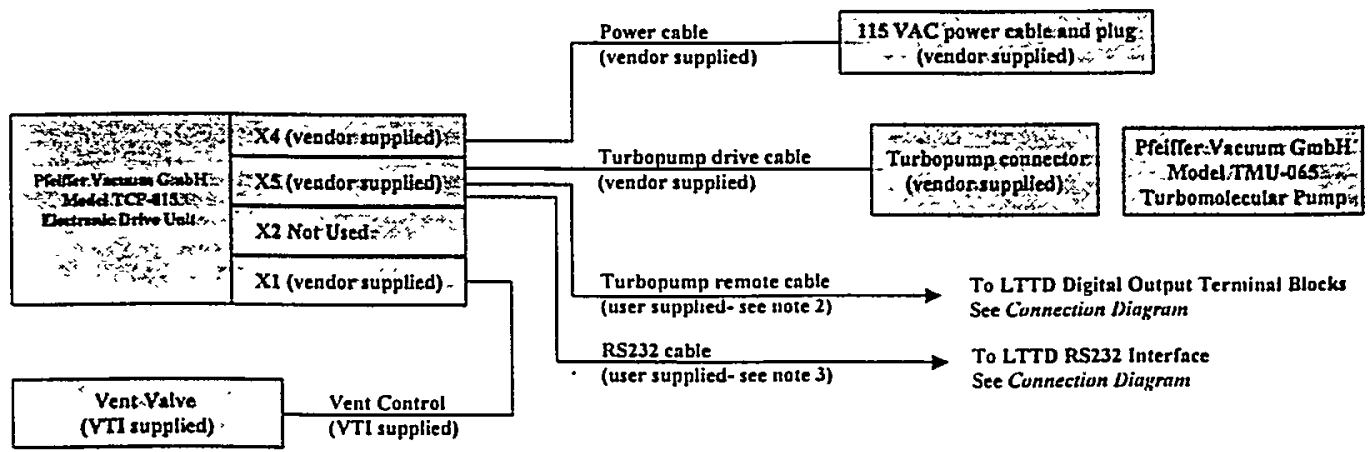

Noce I: Vendor supplied drive eable shall be modilied to provide reset and start control inputs per vendor instruttions - referenee PfeilferVacuum GmbH, Operating Instruetions TCP 015/TCP 035, Section 1.3.1,"Electrical Connections"

Note 2: Turbopump remote control cable shall be 2 shielded twisted pair 24 AWG stranded eable eight feet long. Label one wire "u+" on each pair. Label the other wires "_" on both ends. Label one pair "Shutdow". Label the other pair "Reset". Attath one end of the cable to the vendor supplied connector per the vendor's requirements. Label outer insulation "Turbopump Remote Control "11"

The conneetions are as follows - Shutdown "+" - X5-26 Reset "+" $\quad$-X5-B6

Prepare opposite end of cable for terminal block connections.

Vote 3: Turbopump RS232 cable shall be shielded 3 conductor 22 AWG stranded cable eight feet long. Label the eable "Turbopump RS232 \#23. Altach one end of the cable to a nine pin female type "D" subminature contuector. Lable it COMIt. Attach the other end to the vendor supplied connector per the vendor's requirements.
The connections are as follows - "D" connector
jumper $D$ pins $1-4-6$
jumper D pins 7-8

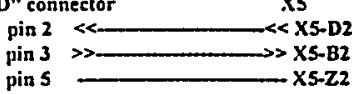


Enhanced Low Temperature Thermal Decomposition (LTTD) Workstation

Cryopump Temperature Display Detail, Rev O, REI 970711

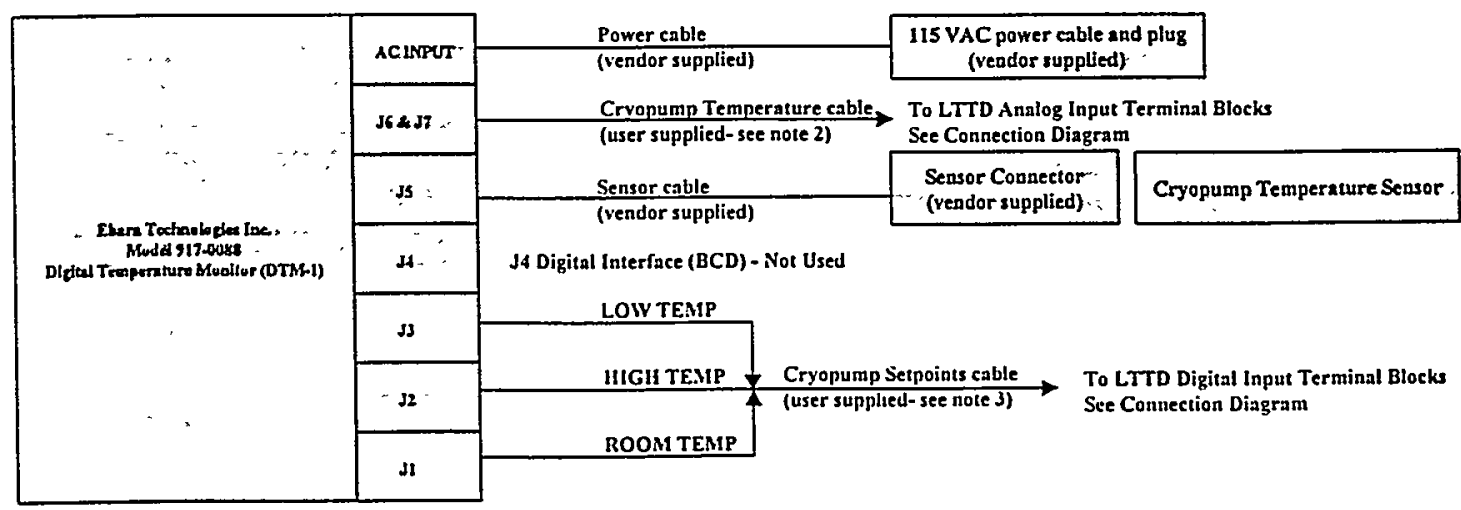

Yote I: User supplied cables for analog and digital outputs stall be constructed per vendor instructions - reference Ebara Technologies Ine. Cryopump Remote Temperature Monitor Operator's Manual. Section 4,"Installation and Operation".

Vote 2: Cryopump Temperature cable shall be shielded twisted pair 20 AWG stranded cable eight feet long. Label "Cryopump Temp. \#13" on both ends outer insulation. Label one wire of the pair " + " on both ends. Label other wire of the pair " of the cable terminate the + and - pairs with red and black bananna plugs respectively. Ternituate the cable shield with the black (-) plug. Prepare opposite end of cable for terminal block connections.

Note 3: Cryopump Setpoints eable shall be three shieided twisted pair 20 or 22 AWG stranded cable eight feet long. Label "Cryopump Setpoints $\# 12$ " on both ends outer iusulation. Label one wire of each pair "COMMON" on both ends. Label other wire of each pair "N.O." on both ends. Label each pair on both ends with "ROOM TEMP". "HIGH TEAIP" and "LOWV TEMP" respectively. On one end terminate each pair with the vendor supplied connectors. For each pair, the "common" wire goes to pin 2 and the N.O. wire goes to pin 1. Prepare opposite end of cable for terminal block connections.

Enhanced Low Temperature Thermal Decomposition (LTTD) Workstation Baratron Display Detail, Rev O, REI 970711

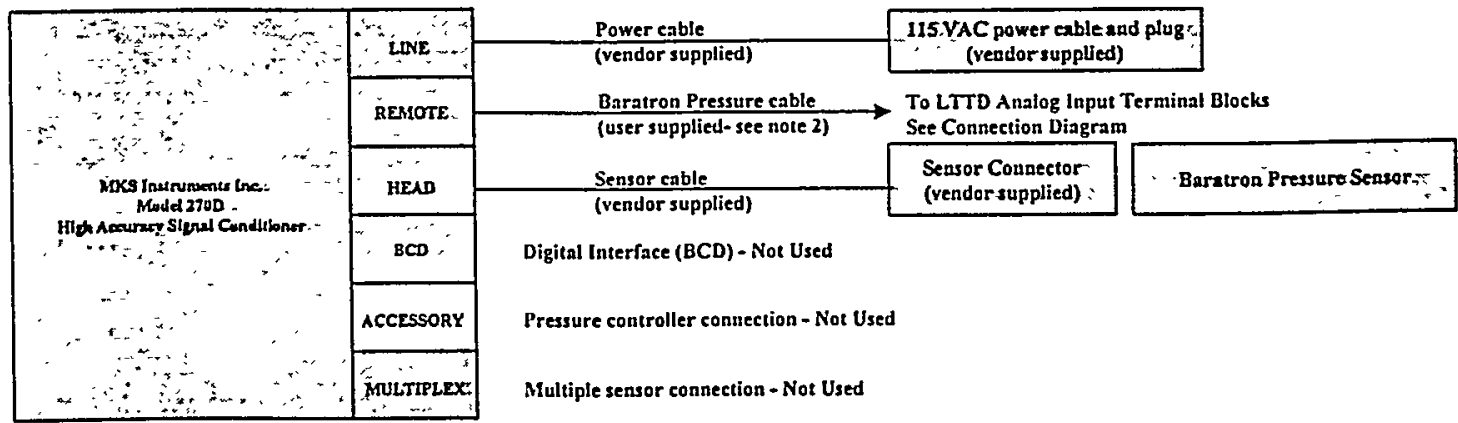

Note 1: User supplied crble for analog outputs shall be construeted per vendor instructions - reference MKS Instruments lnc. Instruction Manual MKS Type 270D High Accuracy Sigaal Conditioner , Chapter 3."Rear Panel Controls".

Note 2: Baratron pressure cable shall be shielded twisted pair 20 AWG stranded eable eight feet long. Label "Baratron Pressure" on both ends outer insulation. Label one wire of the pair "+" on both ends. Label other wire of the pair " the + and - puirs with a 9 pin female type " $D$ " subminature connector. The connections are as follows - "+" - pin I

Prepare opposite end of cable for terminal block connections. shield - pin 6 
Enhanced Low Temperature Thermal Decomposition (LTTD) Workstation Convectron Display Detail, Rev O, REI 970711

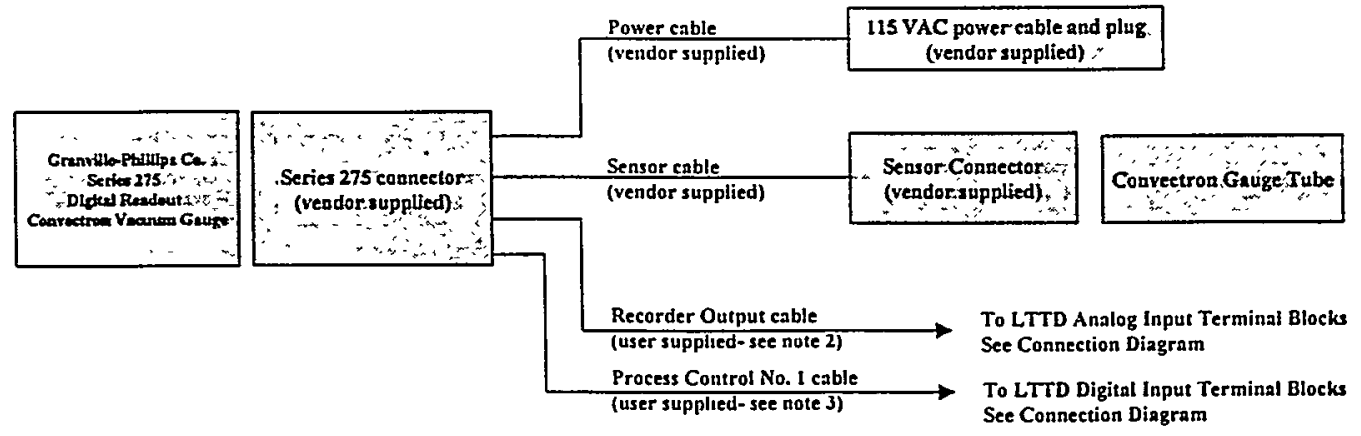

Note t: Vendor supplied cable shall be ntodified to provide Recorder and Process Control Outputs per vendor instructions - reference Granvilie Phillips Series 275 Instruction MIanual. Section 3. "Controller Installation Instructions and Precautions", subsections 2-4

Note 2: Recorder Output eable shall be shielded twisted pair 20 AWG stranded cable eight feet long. Label one wire "Recorder Output" on both ends. Label the other wire "Recorder Return" on both ends. Attach one end of the cable to the vendar supplied connector per the vendor's requirements. The connections are as follows - Recorder Output - pin $M$

Recorder Return - pin $N$

Prepare opposite end of cable for terminal block connections.

$$
\text { shi }
$$

Note 3: Process Control No.1 Relay cable stall be shielded twisted pair 20 AWG stranded cable eight feet long. Label one wire "Process Control No. 1 relay common" on both ends. Label the other wire "Process Control No. 1 relay N.O." on both ends. Attacb one end of the cable to the vendor supplied connector per the vendor's requirements.

$$
\begin{array}{r}
\text { The connections sre as follows - PCIR common } \\
\text { PCIR N.O. } \\
\text { shield } D \\
\end{array}
$$

Prepare opposite end of cable for terminal block connections.

\section{Enhanced Low Temperature Thermal Decomposition (LTTD) Workstation Ion Gauge Display Detail, Rev O, REI 970711}

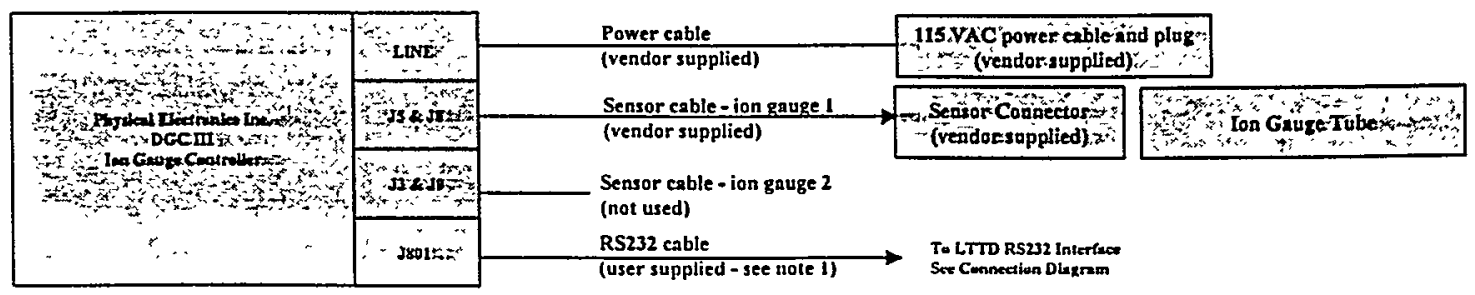

Note 1: Ion gauge RS232 cable shall be shielded 3 conductor 22 AWG stranded cable eight feet long. Label the cable "lon gauge RS232 \$24. Attach one end of the cable to nine pin female type "D" subtminature connector. Lable it COM2. Attach the other end to a 25 pin male type "D" subminature connector. Lable it COMP. INT.

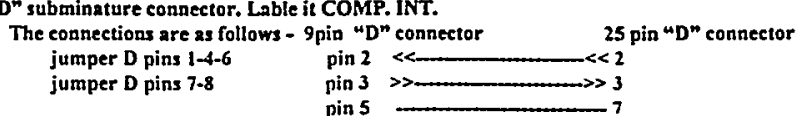


Enhanced Low Temperature Thermal Decomposition (LTTD) Workstation

Manifold Solenoid Valve Detail, Sheet I of 2, Rev O, REI 980210

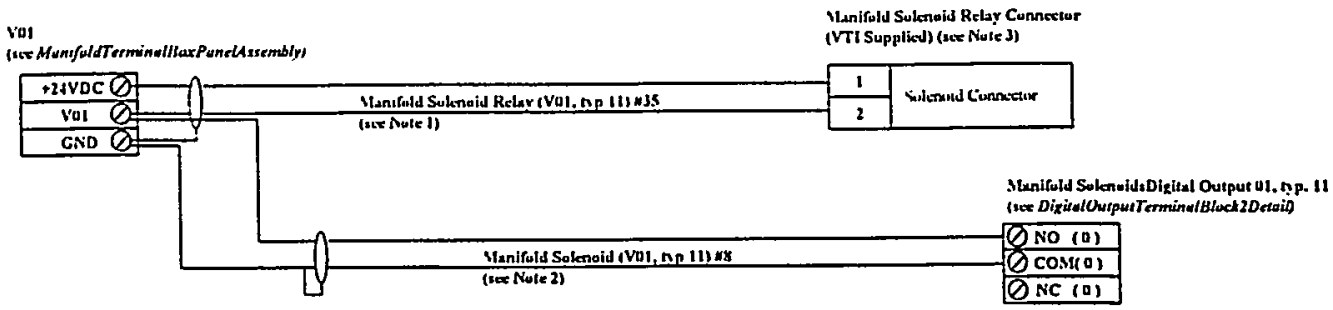

Note 1: Manifold Solenoid Relay cable shall be 15 pair, shielded twisted 24 A WG cable eight feet long. For each pair, Label one wire " $V x x "$ on both ends (where $x x$ is $01-15$ ). Label the other wire "+ $24 V D C$ " on both ends. Label the outer insulation "Vxx" on both ends. Label the entire bundle Manifold Solenoid Relay.

Prepare both ends of each pair for terminal block connections. Wire as shown typieally in above drawing for Vol - VIl. See sheet 2 for remainder of commections. Bind and tape ends of spares (VI4, V15).

Note 2: Munifold Solenoid cable sliall be 15 pair, shielded twisted 24 AWG twenty five feet long. For each pair, Label one wire "Vxx" on both ends (where $x x$ is $01-15$ ). Label the other wire "GND" on both ends. Label tle outer insulation "Vxx" on both ends. Label the entire bundle Manifold Solenoid.

Prepare both ends of each pair for terminal block connections. IVire as slown typically in above drawing for Vol - VII. See sheet 2 for remander of comections. Bind and tape ends of spares (V13.V14.V15).

Yote 3: lustall IN 4004 suppression diodes in the body of the Solenoid connectors. The cathode goes to pin 1 and the anode goes to pin 2.

Enhanced Low Temperature Thermal Decomposition (LTTD) Workstation Manifold Solenoid Valve Detail, Sheet 2 of 2, Rev O, REI 980210

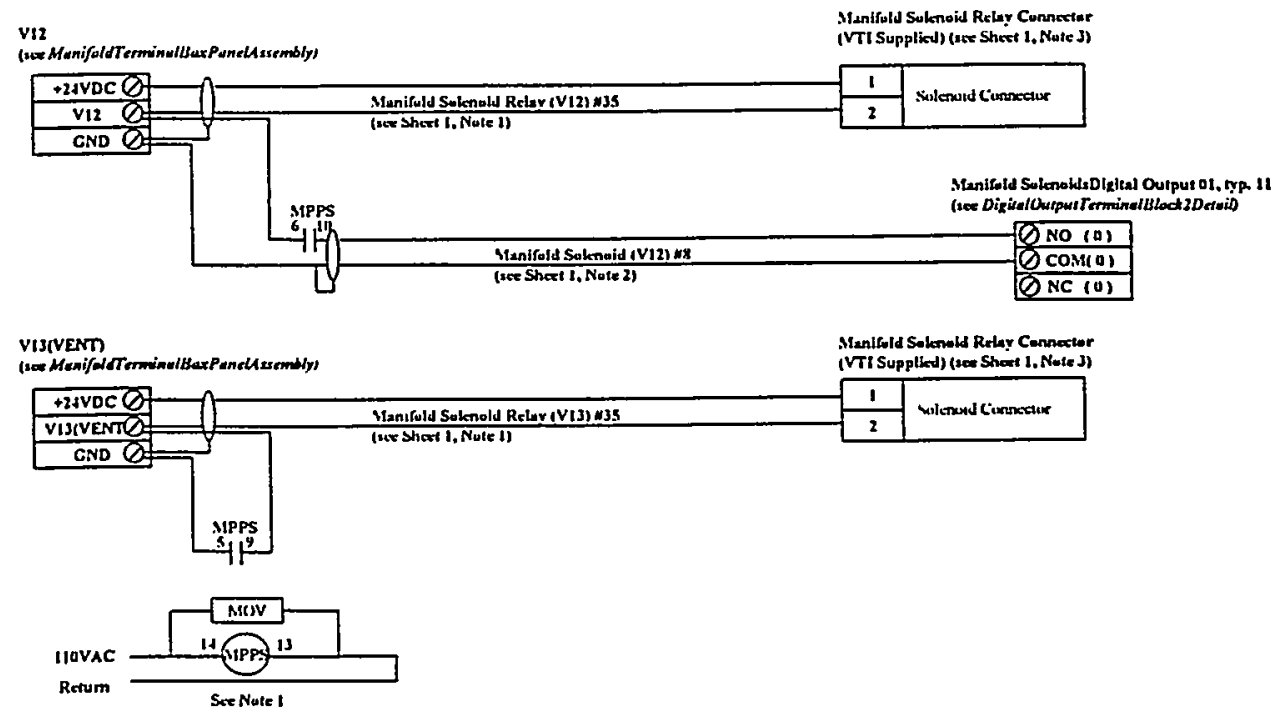

Note I: MenifurTeminullaxPaneLnembly shows the location of the Mechanical Pump Power Sense (MPPS) Relay Socket. This socket is an Allen-Bradley $700 \mathrm{HN} 128$ and is used with an Allen-Bradley $700 \mathrm{HC14AI}$ relay (110VAC coil). Connect the relay and the Siemens S14K130 NOV (or equivalent) as shown in the above drawing. 
Enhanced Low Temperature Thermal Decomposition (LTTD) Workstation

Oven Disable Detail, Sheet I of 2, Rev O, REI 970711

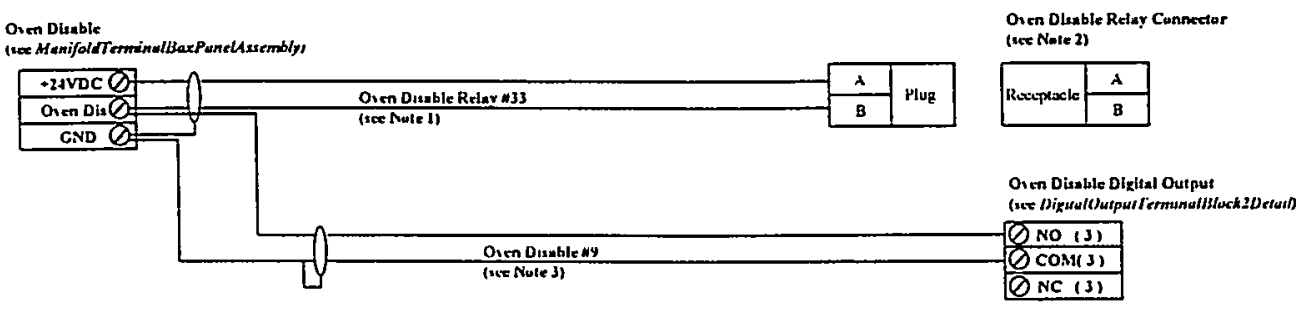

Note 1: Oven Disable Relay cable shall be shielded twisted pair 20 AlvG stranded cable eight feet long. Label one wire "Oven Disable Relay" on both ends. Label the other wire "+ +24 VDC" on both ends. Label the outer insulation "Oven Disable Relay $\# 33^{\prime \prime}$ on both ends. Altach one end of the cable to a ils 3106A-14S-9S plug. The connections are as follows: +24VDC - pin A

Prepare opposite end of cable for terminal block connections.

Oven Disable Relay - piu B

Note 2: Modifications to oven control wiring (Ref: The Grieve Corporation, drawing 069058E).

Mount a MS 3102A-14S-9P receptacle on the oven ter minal box. Mount 2 Potter-Brumfield $27 E 122$ relay socket on the inside of the ove terminal box and install a Potter-Brumfield KRPA-11DG-24 relay. Per manufacturers instructions, use \#16 AlVG stranded wire with red insulation for all control wiring connections. Connector pins A \& B are connected to relay socket pins 2 and 7 respectively. Lobel the new wires M1OD1 and MOD2 respectively. Disconnect wire 7 (near line 14 on the Grieve ladder diagram) from the "ON" contaet of 1 HS. Reconnect wire 7 to pin 1 of the relay socket. Add the label "MIOD3" to wire 7 . Connect the "ON" contact of thS to pin 4 of the relay socket. Label the new wire "MOD4".

Install a 1 $\$ 4004$ power diode on the relay socket with the anode connected to pin 7 and the eathode connected to pin 2.

See sheet 2 of this drawing for additionul detail.

Note 3: Oven Disable cable shall be shielded twisted pair 24 AWG stranded cable tirenty live feet long. Labei one wire "Oven Disable" on both ends. Label the other wire "GND" on both ends. Label the outer insulation "Oven Disable Hy" on both eisds.

Prepare both ends of the cable for terminal block connections.

Enhanced Low Temperature Thermal Decomposition (LTTD) Workstation Oven Disable Detail, Sheet 2 of 2, Rev O, REI 970711

Original wiring re ladder diagram from Grieve drawing

Lise it

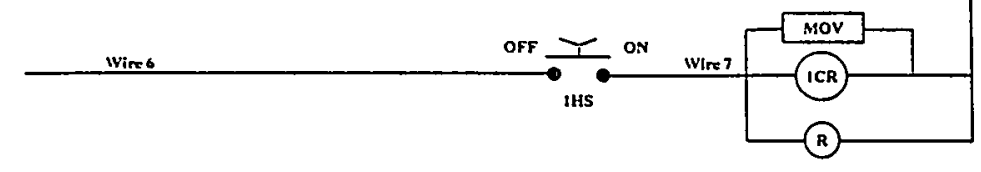

Modified wiring re Note 2 on sheet $!$ of this drawing

Line It

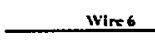

Line is

Line 16 (sex Nale 2 alket I)

Oren Dluble Rdur Rruptuck

\begin{tabular}{|l|l|}
\hline Rocoplesck & $A$ \\
\cline { 2 - 2 }
\end{tabular}

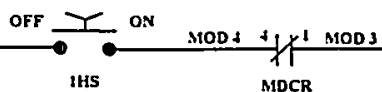

IN+OW 
Enitanced Low Temperature Thermal Decomposition (LTTD) Workstation

Cryopump Compressor Detail, Rev O, REI 970711

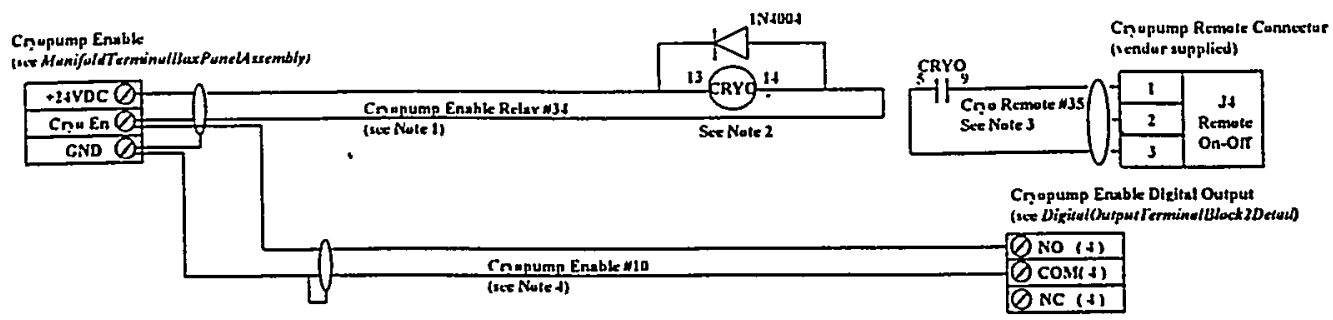

Sote 1: Croypump Enable Relay cable stall be shielded twisted pair 20 AWG stranded cable one foot long. Label one wire "Cryo Enable Relay" on both ends. Label the other wire "+24VDC" on both ends. Label the outer insulation "Cryo Enable Relay *34". Prepare both ends of cable for terminal block connections. Wire as show in above diagram.

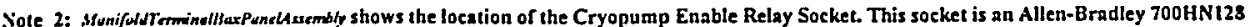
and is used with an Allell-Bradley $700 \mathrm{HCl} 4 \mathrm{Z24}$ relay. Connect the relay and the IN 4004 power diode as shown in the above drawing.

Vote 3: Cryo Remote cable shall be shielded twisted pair 18 AWG stranded eable fifteen feet long. Label one wire "24VAC" on both ends.

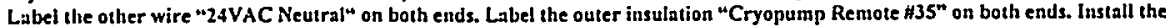
vendor supplied connector on ane end of the enble. The connections are as follows: 24VAC - pin 3 GND $\quad-\operatorname{pin} 2$ 24VAC Neutral - pin 1

Prepare other end of the cable for terminal block connections and connect as shown in the above drawing.

. Note 4: Cryo Enable cable shall be shielded twisted pair 24 AWG stranded cable tirenty five feet long. Label one wire "Cryo Enable" on both ends. Label the other wire "GND" on both ends. Label the outer insulation "Cryopump Enable "10" on both ends.

Prepare both ends of the cable for ter minal block connections and connect as shown in the above drawing.

Enhanced Low Temperature Thermal Decomposition (LTTD) Workstation Can Temperature Sensor Detail, Rev O, REI 970711

Thermecouple
Wedd

Yote I: Can Temperalure Sensor shall be a type J thermocouple (the same sensor used by the oven nanufacturer).

The autalog input clannel shall be connected through type J 20AWG extension grade thermocouple wire (iron:white sheath:t, Constantan:red sheath:-, overall black teflon sheath, $-450-500 \mathrm{~F}$ temperature range). There shall be twenty five feet of extension wire terminated by a lype $\mathrm{J}$ receptacle.

The sensing element shall be a six foot length of Type J 20 AWG thermocouple grade wire (iron:white sheath:+, Constantan:red sheath:-overall brown glass braid. $-100 t 0900 \mathrm{~F}$ (emperature range) welded on the sensing end and (erminated in a type $J$ plug on the opposite end.

Omega part number for a five pack of pre-made six foot type $J$ thermocouples is STC-GG-J-20-72.

Omega part number for a twenty five foot length of type J 20AWG thermocouple extension wire is EXTT-J-20-SB-T-25

Omega part number for type $J$ ther mocouple connectors (plug-receptacie pairs) is OGP-J-MF. 
Enhanced Low Temperature Thermal Decomposition (LTTD) Workstation Manifold Temperature Sensor Detail, Rev O, REI 970711

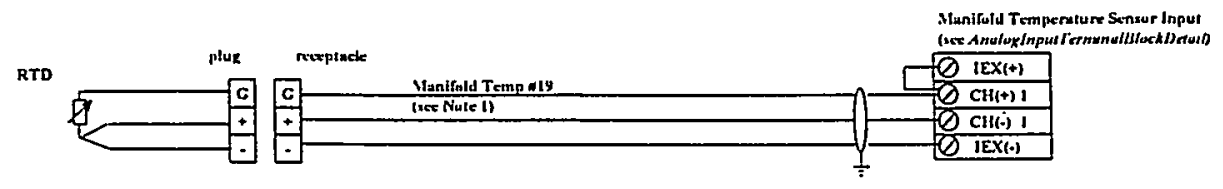

Sote 1: Manifold Temperature Sensor shall be abutton" style RTD.

Omega part number RTD-809 is a "button" style RTD with a connector for user cabling.

User cabling shall be a twenty five foot length of 24 AWG three conductor shielded cable. Lable the outer insulator manifold Temp \#19" on each end. Prepare both ends of all three conductors for screw terminations and connect as shown the above drawing. Connect the shield to the terminal block ground lug. 